\title{
The millimeter IRAM-30 m line survey toward IK Tauri ${ }^{\star} \star \star \star$
}

\author{
L. Velilla Prieto ${ }^{1,2}$, C. Sánchez Contreras ${ }^{2}$, J. Cernicharo ${ }^{1}$, M. Agúndez ${ }^{1}$, G. Quintana-Lacaci ${ }^{1}$, V. Bujarrabal ${ }^{3}$, \\ J. Alcolea ${ }^{4}$, C. Balança ${ }^{5}$, F. Herpin ${ }^{6,7}$, K. M. Menten ${ }^{8}$, and F. Wyrowski ${ }^{8}$
}

\author{
${ }^{1}$ Instituto de Ciencia de Materiales de Madrid, CSIC, c/ Sor Juana Inés de la Cruz 3, 28049 Cantoblanco, Madrid, Spain \\ e-mail: lvelilla@icmm.csic.es \\ 2 Centro de Astrobiología, INTA-CSIC, 28691 Villanueva de la Cañada, Madrid, Spain \\ 3 Observatorio Astronómico Nacional (IGN), Ap 112, 28803 Alcalá de Henares, Madrid, Spain \\ 4 Observatorio Astronómico Nacional (IGN), Alfonso XII No 3, 28014 Madrid, Spain \\ 5 LERMA, Observatoire de Paris, Sorbonne Université, UPMC, UMR 8112, 92195 Meudon, France \\ ${ }^{6}$ Université de Bordeaux, LAB, UMR 5804, 33270 Floirac, France \\ 7 CNRS, LAB, UMR 5804, 33270 Floirac, France \\ ${ }^{8}$ Max-Planck-Institut für Radioastronomie, Auf dem Hügel 69, 53121 Bonn, Germany
}

Received 22 April 2016 / Accepted 24 August 2016

\section{ABSTRACT}

\begin{abstract}
Aims. We aim to investigate the physical and chemical properties of the molecular envelope of the oxygen-rich AGB star IK Tau. Methods. We carried out a millimeter wavelength line survey between $\sim 79$ and $356 \mathrm{GHz}$ with the IRAM-30 m telescope. We analysed the molecular lines detected in IK Tau using the population diagram technique to derive rotational temperatures and column densities. We conducted a radiative transfer analysis of the $\mathrm{SO}_{2}$ lines, which also helped us to verify the validity of the approximated method of the population diagram for the rest of the molecules.

Results. For the first time in this source we detected rotational lines in the ground vibrational state of $\mathrm{HCO}^{+}, \mathrm{NS}, \mathrm{NO}$, and $\mathrm{H}_{2} \mathrm{CO}$, as well as several isotopologues of molecules previously identified, namely, $\mathrm{C}^{18} \mathrm{O}, \mathrm{Si}^{17} \mathrm{O}, \mathrm{Si}^{18} \mathrm{O},{ }^{29} \mathrm{SiS},{ }^{30} \mathrm{SiS}, \mathrm{Si}^{34} \mathrm{~S}, \mathrm{H}^{13} \mathrm{CN},{ }^{13} \mathrm{CS}$, $\mathrm{C}^{34} \mathrm{~S}_{2} \mathrm{H}_{2}{ }^{34} \mathrm{~S},{ }^{34} \mathrm{SO}$, and ${ }^{34} \mathrm{SO}_{2}$. We also detected several rotational lines in vibrationally excited states of $\mathrm{SiS}$ and $\mathrm{SiO}$ isotopologues, as well as rotational lines of $\mathrm{H}_{2} \mathrm{O}$ in the vibrationally excited state $v_{2}=2$. We have also increased the number of rotational lines detected of molecules that were previously identified toward IK Tau, including vibrationally excited states, enabling a detailed study of the molecular abundances and excitation temperatures. In particular, we highlight the detection of $\mathrm{NS}_{\text {and }} \mathrm{H}_{2} \mathrm{CO}$ with fractional abundances of $f(\mathrm{NS}) \sim 10^{-8}$ and $f\left(\mathrm{H}_{2} \mathrm{CO}\right) \sim\left[10^{-7}-10^{-8}\right]$. Most of the molecules display rotational temperatures between 15 and $40 \mathrm{~K}$. $\mathrm{NaCl}$ and $\mathrm{SiS}$ isotopologues display rotational temperatures higher than the average $(\sim 65 \mathrm{~K})$. In the case of $\mathrm{SO}_{2}$ a warm component with $T_{\text {rot }} \sim 290 \mathrm{~K}$ is also detected.

Conclusions. With a total of $\sim 350$ lines detected of 34 different molecular species (including different isotopologues), IK Tau displays a rich chemistry for an oxygen-rich circumstellar envelope. The detection of carbon bearing molecules like $\mathrm{H}_{2} \mathrm{CO}$, as well as the discrepancies found between our derived abundances and the predictions from chemical models for some molecules, highlight the need for a revision of standard chemical models. We were able to identify at least two different emission components in terms of rotational temperatures. The warm component, which is mainly traced out by $\mathrm{SO}_{2}$, is probably arising from the inner regions of the envelope (at $\lesssim 8 R_{*}$ ) where $\mathrm{SO}_{2}$ has a fractional abundance of $f\left(\mathrm{SO}_{2}\right) \sim 10^{-6}$. This result should be considered for future investigation of the main formation channels of this, and other, parent species in the inner winds of O-rich AGB stars, which at present are not well reproduced by current chemistry models.
\end{abstract}

Key words. astrochemistry - line: identification - stars: abundances - stars: AGB and post-AGB - circumstellar matter

\section{Introduction}

Asymptotic giant branch (AGB) stars are the main contributors to the interstellar medium (ISM) chemical enrichment. The physical conditions, that is, the high densities $\left(\gtrsim 10^{12} \mathrm{~cm}^{-3}\right)$ and temperatures $(2000-3000 \mathrm{~K})$, in their atmospheres allow the formation of stable molecules. All this molecular material is driven by the slow AGB wind creating a circumstellar envelope (CSE) that surrounds the star, up to regions where the interstellar UV field photodissociates the molecules. Carbon and oxygen are

\footnotetext{
* Based on observations carried out with the IRAM-30 m Telescope. The Institut de Radioastronomie Millimétrique (IRAM) is supported by INSU/CNRS (France), MPG (Germany) and IGN (Spain).

$\star \star$ Full Tables E.1 and E.2 and the reduced spectrum (FITS file) are only available at the CDS via anonymous ftp to

cdsarc.u-strasbg. fr (130.79.128.5) or via

http://cdsarc.u-strasbg.fr/viz-bin/qcat?J/A+A/597/A25
}

the two most abundant and reactive elements in the atmospheres and winds of AGB stars, after hydrogen. All the possible carbon monoxide, which is a very stable molecule, is formed and then depending on which element (carbon or oxygen) is in excess, other molecules will be formed. Hence, the chemistry in these objects mainly depends on the elemental carbon to oxygen ratio, being O-rich $([\mathrm{C}] /[\mathrm{O}]<1)$, C-rich $([\mathrm{C}] /[\mathrm{O}]>1)$ or S-type stars $([\mathrm{C}] /[\mathrm{O}] \sim 1)$ (e.g. Olofsson 1996).

Since the first detection of $\mathrm{CO}$ in the millimeter wavelength range toward the CSE of an AGB star (Solomon et al. 1971), the observations of the molecular emission of CSEs in that wavelength domain have increased (e.g. Morris et al. 1975; Bujarrabal \& Alcolea 1991; Cernicharo et al. 2015). These studies have been mostly focused on C-rich CSEs given that carbon is more chemically active than oxygen and, therefore, $\mathrm{C}$-rich envelopes are expected and observed to display a large 
variety of different molecular species (e.g. Cernicharo et al 2000; Smith et al. 2015). However, the number of studies in the millimeter wavelength range has increased in the last years, and these studies have evidenced that O-rich CSEs do also host a rich variety of molecules (Ziurys et al. 2007; Kim et al. 2010; De Beck et al. 2013; Sánchez Contreras et al. 2015, and references therein).

Our motivation is to observe and to study the molecular content of the oxygen-rich CSE IK Tauri which is one of the most studied O-rich CSEs, and is considered a reference of its class.

\subsection{IK Tauri}

IK Tauri (hereafter IK Tau), also known as NML Tau, is a Mira-type variable star with a period of 470 days and a spectral type M9 (Pesch 1967; Wing \& Lockwood 1973; Alcolea et al. 1999). This star was discovered by Neugebauer et al. (1965) and it is located at $\alpha(\mathrm{J} 2000)=3^{\mathrm{h}} 53^{\mathrm{m}} 28.87$ and $\delta(\mathrm{J} 2000)=$ $11^{\circ} 24^{\prime} 21^{\prime \prime} 7$ (Cutri et al. 2003). The distance to IK Tau was estimated to be 250-265 pc (Hale et al. 1997; Olofsson et al. 1998). The systemic velocity of the star with respect to the local standard of rest is $V_{\text {sys }}^{\mathrm{LSR}} \sim 34 \mathrm{~km} \mathrm{~s}^{-1}$ (Kim et al. 2010, and references therein). Its effective temperature is $T_{\text {eff }} \sim 2200 \mathrm{~K}$ and the stellar radius is $R_{*} \sim 2.5 \times 10^{13} \mathrm{~cm}$ (Decin et al. 2010a).

The star is surrounded by a an O-rich CSE, composed of dust and gas, which is the result of mass loss at a rate that has been estimated by different methods. Neri et al. (1998) estimated $\dot{M} \sim 3.8 \times 10^{-6} M_{\odot} \mathrm{yr}^{-1}$ from the model of ${ }^{12} \mathrm{CO} J=1-0$ and $J=$ 2-1 lines. González Delgado et al. (2003) estimated $\dot{M} \sim 3.0 \times$ $10^{-5} M_{\odot} \mathrm{yr}^{-1}$ from the model of ${ }^{12} \mathrm{CO} J=1-0, J=2-1, J=3-$ 2 , and $J=4-3$ lines. Recent modelling of the $J=3-2, J=4$ 3 , and $J=7-6$ lines of ${ }^{12} \mathrm{CO}$ yielded $\dot{M} \sim 4.7 \times 10^{-6} M_{\odot} \mathrm{yr}^{-1}$ (Kim et al. 2010). The terminal expansion velocity of the CSE is $v_{\infty} \sim 18.5 \mathrm{~km} \mathrm{~s}^{-1}$ as measured from De Beck et al. (2013) and references therein. The size of the CSE depends on the molecule used to trace it out. Bujarrabal \& Alcolea (1991) gave a halfintensity diameter of $\theta_{1 / 2} \sim 16-17^{\prime \prime}$ measured for ${ }^{12} \mathrm{CO} \mathrm{J}=$ $1-0$ and ${ }^{12} \mathrm{CO} J=2-1$ emission detected with the IRAM$30 \mathrm{~m}$ telescope. Kim et al. (2010) measured a $\theta_{1 / 2} \sim 20^{\prime \prime}$ for ${ }^{12} \mathrm{CO} J=3-2$ with the Atacama Pathfinder EXperiment (APEX) telescope. Recent observations with the Plateau de Bure Interferometer (PdBI), showed that ${ }^{12} \mathrm{CO}(J=1-0)$ displays a $\theta_{1 / 2} \sim 18^{\prime \prime}$ (Castro-Carrizo et al. 2010). Also, HCN $J=1-0$ was observed with the Owens Valley millimeter-array by Marvel (2005), displaying a size of $\theta_{1 / 2} \sim 3$.' 85 . The size of the $\mathrm{SiO} v=0$ $J=2-1$ emission is $\theta_{1 / 2}=2 .{ }^{\prime \prime} 2 \pm 0 . \prime 1$ as determined with PdBI (Lucas et al. 1992). Finally, the emission of several lines of PN and PO has been mapped with the SubMillimeter Array (SMA), with $\theta_{1 / 2} \lesssim 00^{\prime \prime} 65$ (De Beck et al. 2013).

The O-rich CSE around IK Tau displays maser emission of $\mathrm{OH}, \mathrm{H}_{2} \mathrm{O}$, and $\mathrm{SiO}$ (Lane et al. 1987; Bowers et al. 1989; Alcolea \& Bujarrabal 1992; Kim et al. 2010, and references therein). It also shows thermal molecular emission of ${ }^{12} \mathrm{CO},{ }^{13} \mathrm{CO}, \mathrm{SiO},{ }^{29} \mathrm{SiO},{ }^{30} \mathrm{SiO}, \mathrm{OH}, \mathrm{SiS}, \mathrm{HCN}, \mathrm{HC}_{3} \mathrm{~N}, \mathrm{H}_{2} \mathrm{~S}$, $\mathrm{SO}, \mathrm{SO}_{2}, \mathrm{NaCl}, \mathrm{H}_{2} \mathrm{O}, \mathrm{H}_{2}{ }^{17} \mathrm{O}, \mathrm{H}_{2}{ }^{18} \mathrm{O}, \mathrm{NH}_{3}, \mathrm{CS}, \mathrm{CN}, \mathrm{PO}, \mathrm{PN}$, AlO, and tentatively, HNC (Lindqvist et al. 1988; Omont et al. 1993; Bujarrabal et al. 1994; Milam et al. 2007; Kim et al. 2010; Decin et al. 2010b; Menten et al. 2010; Justtanont et al. 2012; De Beck et al. 2013, 2015, and references therein). Some of the molecules observed up to date in IK Tau have been compared with chemical models and their line emission analysed with radiative transfer models (Willacy \& Millar 1997; Duari et al. 1999; Cherchneff 2006; Kim et al. 2010; Decin et al. 2010a; Danilovich et al. 2016; Gobrecht et al. 2016; Li et al. 2016).
Nevertheless, most of the reported abundances in previous studies were derived from the analysis of a moderated number of lines (e.g. Milam et al. 2007; De Beck et al. 2013). Additionally, discrepancies remain between the predicted abundances and the observations, for example for $\mathrm{SO}_{2}$ (Decin et al. 2010a; Gobrecht et al. 2016).

\subsection{This paper}

In this article we report the millimeter wavelength survey between $\sim 79$ and $\sim 356 \mathrm{GHz}$ carried out with the IRAM-30 m telescope toward IK Tau, which allowed us to detect rotational lines of 34 different species (including isotopologues). We detected for the first time in this source $\mathrm{HCO}^{+}, \mathrm{NS}, \mathrm{H}_{2} \mathrm{CO}$, and $\mathrm{NO}$, as well as several isotopologues of previously identified molecules, such as $\mathrm{C}^{18} \mathrm{O}, \mathrm{Si}^{17} \mathrm{O}, \mathrm{Si}^{18} \mathrm{O},{ }^{29} \mathrm{SiS},{ }^{30} \mathrm{SiS}, \mathrm{Si}^{34} \mathrm{~S}, \mathrm{H}^{13} \mathrm{CN},{ }^{13} \mathrm{CS}$, $\mathrm{C}^{34} \mathrm{~S}, \mathrm{H}_{2}{ }^{34} \mathrm{~S},{ }^{34} \mathrm{SO}$, and ${ }^{34} \mathrm{SO}_{2}$. For molecules with previous detections reported in the literature, we increased significantly the number of transitions observed, which is needed for a robust estimate of the excitation conditions and molecular abundances. We report the results of our analysis based on population diagrams, used to derive rotational temperatures and column densities. We also estimated fractional abundances which have been compared with values derived from previous observations and with predictions by chemical models. In the particular case of $\mathrm{SO}_{2}$, we performed a radiative transfer calculation to study the excitation conditions of the $\sim 90$ lines detected with more detail. This suggests that this molecule is rather abundant not only in the intermediate and outer envelope but also in the inner, hotter and more dense regions of the CSE, where we estimate an average fractional abundance of $\sim 10^{-6}$ (with respect to $\mathrm{H}_{2}$ ). This radiative transfer model also allowed us to verify the validity of the population diagram analysis for the rest of the species detected.

\section{Observations}

The observations presented in this paper correspond to a sensitive millimeter-wavelength $(\sim 79-356 \mathrm{GHz})$ survey carried out with the IRAM-30 m telescope toward the CSEs of two O-rich evolved stars, IK Tau and $\mathrm{OH} 231.8+4.2$, during several observing runs from 2009 to 2014. Partial results from the survey toward OH 231.8+4.2 are reported in Sánchez Contreras et al. (2014, 2015) and Velilla Prieto et al. (2013, 2015).

We used the heterodyne Eight MIxer Receiver (EMIR) working at four different wavelengths bands: E090 $=3 \mathrm{~mm}$, $\mathrm{E} 150=2 \mathrm{~mm}, \mathrm{E} 230=1.3 \mathrm{~mm}$, and $\mathrm{E} 330=0.9 \mathrm{~mm}$ (Carter et al. 2012). This receiver system was operated in single-sideband (SSB) mode for the E150 band and in dual sideband (2SB) mode for bands E090, E230, and E330. Two polarizations - horizontal and vertical - were available per sideband. The typical EMIR value image band rejection is approximately $-14 \mathrm{~dB}$ or better, this implies that the peak intensity of a line entering through the image band is only $\$ 4 \%$ of its real value. We verified this value for the image band rejection measuring the relative intensities of strong lines that appear both in the signal and image bands.

Four different backends or spectrometers were connected to each receiver depending on their availability: the WIde Lineband Multiple Autocorrelator (WILMA), the fast Fourier Transform Spectrometer (FTS), the VErsatile SPectrometer Array (VESPA) and the $4 \mathrm{MHz}$ spectrometer. The capabilities of these spectrometers and their usage are summarised in Table 1.

The observational technique used was the wobbler switching with a single pointing toward the position of the source (see 
Table 1. Specifications for the spectrometers used.

\begin{tabular}{lcccc}
\hline \hline Name & $\begin{array}{c}\text { SR } \\
-\end{array}$ & $\begin{array}{c}\text { VR } \\
(\mathrm{MHz})\end{array}$ & $\begin{array}{c}\text { IBWEP } \\
\left(\mathrm{km} \mathrm{s}^{-1}\right)\end{array}$ & $\begin{array}{c}\text { Usage } \\
(\mathrm{GHz})\end{array}$ \\
\hline WILMA & 2.000 & $1.7-7.6$ & 3.7 & E090, E150, E230, E330 \\
FTS & 0.195 & $0.2-0.7$ & 4.0 & E230, E330 \\
VESPA & 1.250 & $1.0-4.7$ & 0.4 & E090, E150, E230, E330 \\
4 MHz & 4.000 & $3.4-15.2$ & 4.0 & E150, E230, E330 \\
\hline
\end{tabular}

Notes. Column 2: spectral resolution; Col. 3: spectral resolution in velocity units, the highest velocity resolution (the first number of the range) corresponds to $356 \mathrm{GHz}$ and the lowest resolution to $79 \mathrm{GHz}$; Col. 4: instantaneous bandwidth in each polarization; Col. 5: EMIR bands observed with the corresponding spectrometer.

Sect.1.1) and a wobbler throw of $120^{\prime \prime}$ in azimuth. We configured different setups (tuning steps) to observe both polarizations simultaneously until we covered the total frequency range available for each EMIR band. We selected a small overlap between adjacent setups to ensure a uniform calibration across the bands. During the observations we checked regularly the pointing and focus of the antenna every $\sim 1.5$ and $\sim 4 \mathrm{~h}$, respectively, on strong nearby sources. On-source integration times per setup were $\sim 1 \mathrm{~h}$. Calibration scans on the standard two loads + sky system were taken every $\sim 18$ min using the atmospheric transmission model (ATM) adopted by IRAM-30 m (Cernicharo et al. 1985; Pardo et al. 2001). Errors in the absolute flux calibration are expected to be $\$ 25 \%$.

The data reduction, analysis and also most of the graphic representation were done using the GILDAS ${ }^{1}$ software package. The standard procedure followed to reduce the data and obtain the final spectra consists of the flagging of bad channels, the flagging of low-quality scans, the baseline substracting, and finally, the averaging of individual scans.

The output spectra obtained from the antenna are calibrated in antenna temperature $\left(T_{\mathrm{A}}{ }^{*}\right)$, which can be converted to main beam temperature $\left(T_{\mathrm{mb}}\right)$ and brightness temperature $\left(T_{\mathrm{B}}\right)$ according to:

$$
\begin{aligned}
T_{\mathrm{B}} & =T_{\mathrm{A}}^{*} \eta^{-1} b f f^{-1}, \\
\eta^{-1} & =F_{\mathrm{eff}} / B_{\mathrm{eff}}, \\
b f f^{-1} & =\left(\theta_{\mathrm{b}}^{2}+\theta_{\mathrm{s}}^{2}\right) / \theta_{\mathrm{s}}^{2},
\end{aligned}
$$

where $\eta$ is the main beam efficiency, $b f f$ is the beam-filling factor, $B_{\text {eff }}$ is the main-beam efficiency of the antenna, $F_{\text {eff }}$ is the forward efficiency of the antenna, $\theta_{\mathrm{s}}$ is the size (diameter) of the emitting region of the source, and $\theta_{\mathrm{b}}$ is the half power beamwidth (HPBW) of the main beam of the antenna. See Table 2 for a summary of the relevant telescope parameters.

The half power width of the main beam can be approximated, using the values in Table 2, to a good accuracy by the expression:

$$
\theta_{\mathrm{b}}\left({ }^{\prime \prime}\right)=2460 / v(\mathrm{GHz})
$$

and the inverse of the main beam efficiency can be fitted (see Fig. 1) using the parameters in Table 2, to obtain:

$\eta^{-1} \equiv F_{\text {eff }} / B_{\text {eff }}=1.114 \exp \left\{(v(\mathrm{GHz}) / 399.5)^{2}\right\}$.

1 GILDAS is a world-wide software to process, reduce and analyse astronomical single-dish and interferometric observations mainly. It is maintained by the Institut de Radioastronomie Millimétrique (IRAM). See http://www.iram.fr/IRAMFR/GILDAS
Table 2. Main parameters of the IRAM-30 $\mathrm{m}$ antenna measured with EMIR between 2009 and 2013 at representative frequencies.

\begin{tabular}{ccccc}
\hline \hline $\begin{array}{c}\text { Frequency } \\
(\mathrm{GHz})\end{array}$ & $\begin{array}{c}B_{\text {eff }} \\
(\%)\end{array}$ & $\begin{array}{c}F_{\text {eff }} \\
(\%)\end{array}$ & $\begin{array}{c}\mathrm{HPBW} \\
\left({ }^{\prime \prime}\right)\end{array}$ & $\begin{array}{c}\mathrm{S} / \mathrm{T}_{A}^{*} \\
(\mathrm{Jy} / \mathrm{K})\end{array}$ \\
\hline 86 & 81 & 95 & $29^{\dagger}$ & $5.9^{\dagger}$ \\
115 & 78 & 94 & - & - \\
145 & 73 & 93 & $16^{\dagger}$ & $6.4^{\dagger}$ \\
210 & 63 & 94 & $11^{\dagger}$ & $7.5^{\dagger}$ \\
230 & 59 & 92 & $10.7^{\dagger}$ & - \\
280 & 49 & 87 & - & - \\
340 & 35 & 81 & $7.5^{\dagger}$ & $10.9^{\dagger}$ \\
345 & 34 & 80 & - & - \\
\hline
\end{tabular}

Notes. Column 1: representative frequency; Col. 2: beam efficiency; Col. 3: forward efficiency; Col. 4: half power beam width; Col. 5: flux density to antenna temperature conversion factor in Jansky per Kelvin for a point-like source. ${ }^{(\dagger)} 2009$ values. ${ }^{(\ddagger)} 2012$ value. The values without symbols were reported on 2013. Source: http://wwW.iram.es/ IRAMES/mainWiki/Iram30mEfficiencies

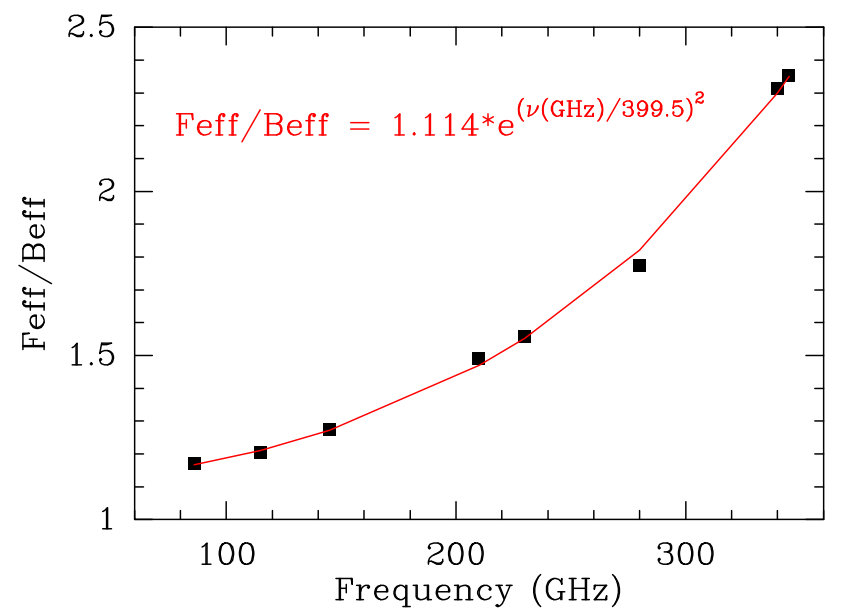

Fig. 1. Fit of the inverse of the main beam efficiency for the IRAM-30 m with EMIR.

\subsection{Observational results}

Our results are based mainly on the spectra obtained with WILMA and FTS given their better spectral resolution and bandwidth. The data obtained with the $4 \mathrm{MHz}$ spectrometer were used to check the edges of the setups that were only observed with WILMA, given that the $4 \mathrm{MHz}$ bandwidth is slightly larger than the bandwidth of WILMA, but it has the same bandwidth than that of the FTS (see Table 1). VESPA data were used only to check certain line profiles. However, only WILMA was available to cover the full EMIR wavelength range. For example, FTS was not avaliable in the E150 band at the epoch of the observations and also some technical issues prevented us from use the FTS with the E090 band.

The spectra of the full mm-wavelength survey carried out with IRAM-30 m toward IK Tau with the WILMA and the FTS spectrometers can be seen in Fig. 2 and also in more detail in Fig. C.1. The ${ }^{12} \mathrm{CO},{ }^{13} \mathrm{CO}$, and $\mathrm{C}^{18} \mathrm{O}$ line profiles are shown in Fig. 3. In Table 3 we show a summary of the observational results. 


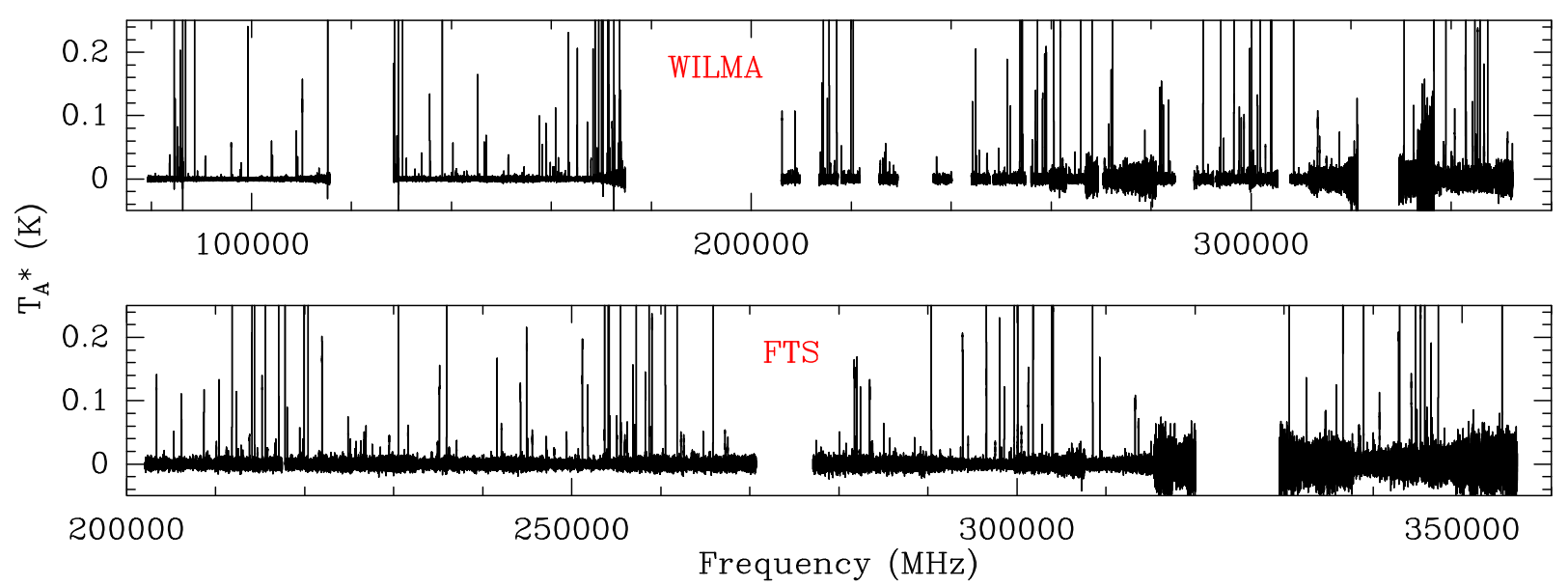

Fig. 2. Overall view of the survey observed with WILMA (top) and FTS (bottom). The spectral resolution is $\Delta v=2 \mathrm{MHz}$.

Table 3. Summary of the observational results.

\begin{tabular}{ccccc}
\hline \hline $\begin{array}{c}\text { Band } \\
-\end{array}$ & $\begin{array}{c}\text { SP } \\
-\end{array}$ & $\begin{array}{c}v_{\text {obs }} \\
(\mathrm{GHz})\end{array}$ & $\begin{array}{c}\text { rms } \\
(\mathrm{mK})\end{array}$ & $\begin{array}{c}\text { Opacity } \\
-\end{array}$ \\
\hline E090 & WILMA & $79.3-115.7$ & $1.5(0.5)$ & $0.12(0.05)$ \\
E150 & WILMA & $128.4-174.8$ & $2.7(1.3)$ & $0.19(0.11)$ \\
E230 & FTS & $202.1-270.7^{\dagger}$ & $4.7(0.6)$ & $0.18(0.03)$ \\
E330 & FTS & $277.1-356.2^{\ddagger}$ & $12.8(10.4)$ & $0.26(0.16)$ \\
\hline
\end{tabular}

Notes. Values given between parentheses represent $1 \sigma$ of the value. Col. 1 EMIR band; Col. 2 spectrometer; Col. 3 observed frequency windows in GHz; Col. 4 root mean square (rms) noise in units of $T_{\mathrm{a}}^{*}$ for a spectral resolution of $2 \mathrm{MHz}$; Col. 5 zenith atmospheric opacity at the observed frequency. ${ }^{(\dagger)} 217.5-217.8$ gap. ${ }^{(\ddagger)} 325.0-329.5$ gap.

\section{Line identification}

For the line identification, we used the public line catalogues from the Cologne Database for Molecular Spectroscopy (CDMS, Müller et al. 2005) and the Jet Propulsion Laboratory (JPL, Pickett et al. 1998), together with a private spectroscopic catalogue that assembles information for more than five thousand spectral entries (atoms and molecules, including vibrationally excited states), compiled from extensive laboratory and theoretical works (the MADEX code, Cernicharo 2012).

Given the wavelength range covered in our observations, we detected, mainly, rotational transitions in the ground vibrational state, but also, rotational transitions in higher vibrational states (i.e. $\mathrm{SiO} v=1, \mathrm{SiO} v=2, \mathrm{SiO} v=3,{ }^{29} \mathrm{SiO} v=1,{ }^{29} \mathrm{SiO} v=2$, ${ }^{29} \mathrm{SiO} v=3,{ }^{30} \mathrm{SiO} v=1,{ }^{30} \mathrm{SiO} v=2, \mathrm{H}_{2} \mathrm{O} v_{2}=1$, and $\mathrm{H}_{2} \mathrm{O} \quad v_{2}=2$ ). We established the detection limit at $\geq 5 \sigma$ with respect to the integrated intensity for well detected features, and between $3 \sigma$ and $5 \sigma$ for tentative detections, although, these tentative detections may be more reliable when they belong to wellknown species that have been identified through other stronger lines.

We detected $\sim 450$ spectral features in the spectra of IK Tau, $\sim 90 \%$ of which are lines in the signal side band of the receivers. Of the $\sim 400$ signal band features, $\sim 350$ lines have been unambiguously identified with rotational transitions of 34 different species (including vibrationally excited states), which are reported in Tables A.1 and A.2, along with some of their spectroscopic parameters and their fitted parameters. The rest of the lines $(\sim 35)$ remain unidentified, although, we proposed a tentative identification for some of them (see Table B.1).

There were several lines that we assigned to spurious features produced in the receivers in the range $167475-174800 \mathrm{MHz}$
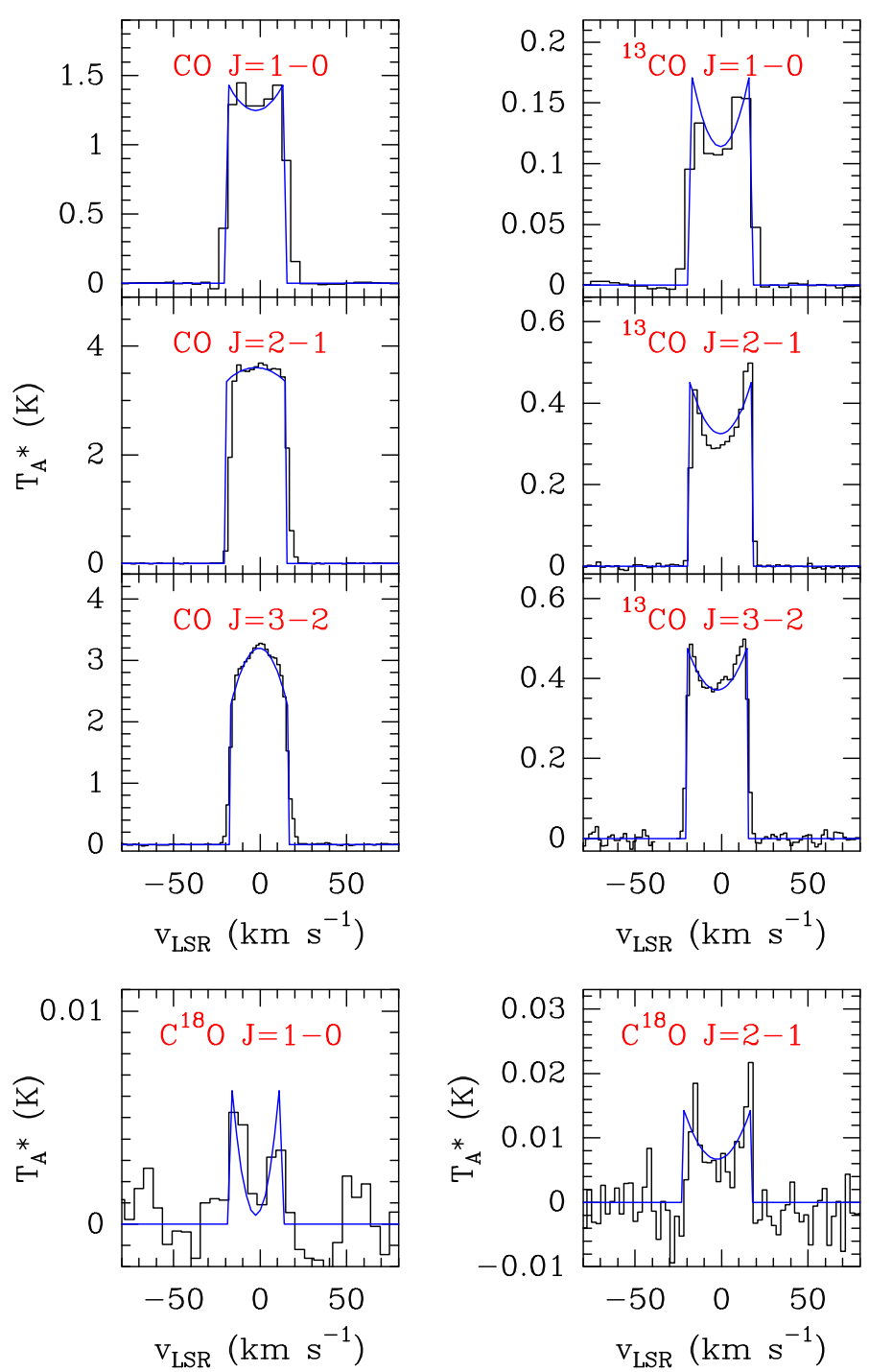

Fig. 3. ${ }^{12} \mathrm{CO},{ }^{13} \mathrm{CO}$, and $\mathrm{C}^{18} \mathrm{O}$ lines detected with our millimeter wavelength survey with a spectral resolution of $\Delta v=2 \mathrm{MHz}$. The fit of each line to a function of the type given by Eq. (6) is shown in blue.

(Table B.2). These spurious features are symmetrical replicas at both sides of certain strong real emission lines, with the intensities of the replicas decreasing with the frequency distance to the real feature. We show in Fig. B.1 an example of this problem. 
The features of the image band and the spurious features were blanked in the final data.

We identified for the first time toward IK Tau, rotational lines of: $\mathrm{HCO}^{+}, \mathrm{NO}, \mathrm{H}_{2} \mathrm{CO}, \mathrm{NS}, \mathrm{C}^{18} \mathrm{O}, \mathrm{SiO} v=2,{ }^{29} \mathrm{SiO} v=2$, ${ }^{29} \mathrm{SiO} v=3,{ }^{30} \mathrm{SiO} v=2, \mathrm{Si}^{17} \mathrm{O}, \mathrm{Si}^{18} \mathrm{O},{ }^{29} \mathrm{SiS},{ }^{30} \mathrm{SiS}, \mathrm{Si}^{34} \mathrm{~S}$, SiS $v=1, \mathrm{H}^{13} \mathrm{CN},{ }^{13} \mathrm{CS}, \mathrm{C}^{34} \mathrm{~S}, \mathrm{H}_{2}{ }^{34} \mathrm{~S},{ }^{34} \mathrm{SO},{ }^{34} \mathrm{SO}_{2}$, and $\mathrm{H}_{2} \mathrm{O} v_{2}=2$. We also detected rotational lines of: ${ }^{12} \mathrm{CO},{ }^{13} \mathrm{CO}$, $\mathrm{SiO}, \mathrm{SiO} v=1, \mathrm{SiO} v=3,{ }^{29} \mathrm{SiO},{ }^{29} \mathrm{SiO} v=1,{ }^{30} \mathrm{SiO} v=1$, $\mathrm{SiS}, \mathrm{HCN}, \mathrm{HNC}, \mathrm{CS}, \mathrm{NaCl}, \mathrm{H}_{2} \mathrm{~S}, \mathrm{SO}, \mathrm{SO}_{2}, \mathrm{PN}, \mathrm{PO}, \mathrm{CN}, \mathrm{H}_{2} \mathrm{O}$, and $\mathrm{H}_{2} \mathrm{O} v_{2}=1$. The result of the identification can be seen in Fig. C.1.

\section{Data analysis}

\subsection{Line profiles}

Most of the lines show profiles that can be reasonably well fitted with the so-called shell profile provided by the software CLASS $^{2}$ :

$f(v)=\frac{A}{\Delta v} \frac{1+4 H\left[\left(v-v_{\mathrm{o}}\right) / \Delta v\right]^{2}}{1+H / 3}$,

where $A$ is the area under the profile, $v_{\mathrm{o}}$ is the central frequency, $\Delta v$ is the full width at zero intensity, and $H$ is the horn to centre ratio. The expansion velocity $\left(v_{\text {exp }}\right)$ can be related to the $\Delta v$ of a line through the expression:

$v_{\exp }=c \frac{\Delta v / 2}{v_{\mathrm{o}}}$,

where $c$ is the speed of light.

In the case of a spherical CSE, there are several typical line profiles which are commonly found: U-shaped, parabolic, flattopped, Gaussian-like or triangular. Each type of profile has a particular interpretation in terms of the size of the emitting region compared to the $\theta_{\mathrm{b}}$ of the telescope, the optical thickness of the line, and the kinematical properties of the gas responsible of the spectroscopic feature (e.g. Zuckerman 1987; Habing \& Olofsson 2004). All these profiles are described to a good accuracy by the shell function described before in Eq. (6).

In Fig. 4 we show a sample of the line profiles observed. Most of the lines display profiles that match one of the types mentioned before, although, some profiles are complex and have to be considered carefully in the analysis. We also observed lines that are significatively narrower and more intense than the average, that is, they show maser like spectral profiles (see Fig. 4 bottom-right panel). For the sake of consistency we used the shell profile described in Eq. (6) to fit all the lines detected, even for the narrow lines since that should give us an approximated idea of the characteristic velocity in the regions of the wind acceleration. The aim of the fit is to estimate the centroids and linewidths, however, the velocity integrated intensities given in Tables A.1, A.2, and B.1 were obtained integrating the whole line profiles. The line profiles observed are discussed in detail for each detected molecule in Sect. 6.

\subsection{Population diagrams and fractional abundances}

Using the population diagram technique (Goldsmith \& Langer 1999), we derived rotational temperatures and column densities averaged in the emitting region of the molecules detected. These values were derived using the following equation:

$\ln \left(\frac{N_{\mathrm{u}}}{g_{\mathrm{u}}}\right)=\ln \left(\frac{3 k_{B} W}{8 \pi^{3} v S_{\mathrm{ul}} \mu^{2}}\right)=\ln \left(\frac{N}{Z}\right)-\frac{E_{\mathrm{u}}}{k_{\mathrm{B}} T_{\mathrm{rot}}}$,

\footnotetext{
2 See http://www.iram. fr/IRAMFR/GILDAS
}
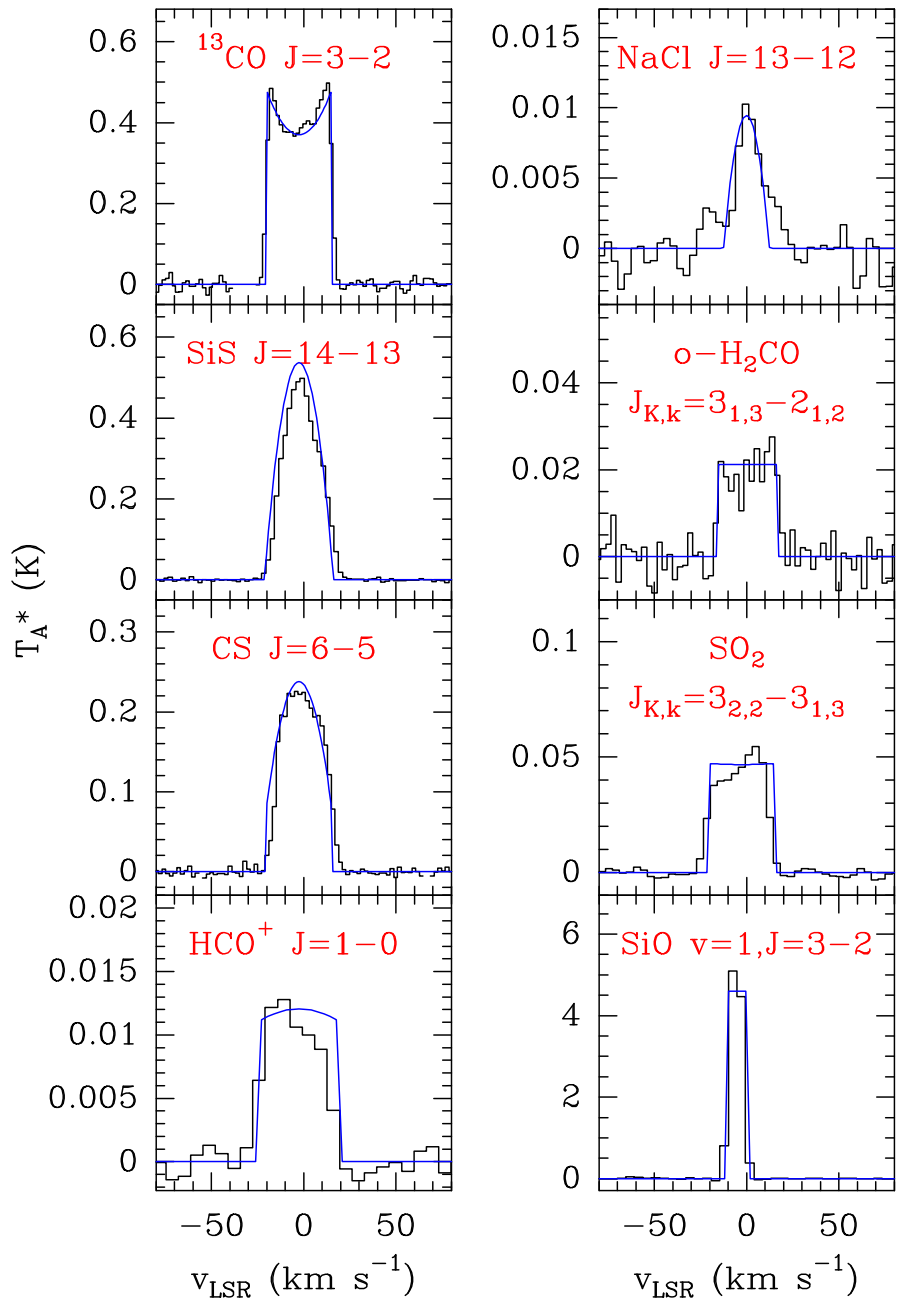

Fig. 4. Representative profiles of the lines observed. From topleft to bottom-right: U-shaped, Gaussian-like, triangular, flat-topped, parabolic, complex, flat-topped, and a narrow maser-like line. The spectral resolution is $\Delta v=2 \mathrm{MHz}$. The fit of each line to a function of the type given by Eq. (6) is shown in blue.

where $N_{\mathrm{u}}$ is the column density of the upper level, $g_{\mathrm{u}}$ is the degeneracy of the upper level, $W$ is the velocity-integrated intensity of the line, $k_{\mathrm{B}}$ is the Boltzmann constant, $v$ is the rest frequency of the transition, $S_{\mathrm{ul}}$ is the line strength, $\mu$ is the dipole moment of the molecule, $N$ is the total column density, $Z$ is the partition function, $E_{\mathrm{u}}$ is the upper level energy of the transition, and $T_{\text {rot }}$ is the rotational temperature. The results of the populations diagrams are reported in Sect. 5.2 and Appendix D.

This method relies on local thermodynamical equilibrium (LTE), optically thin emission, as well as an uniform rotational temperature for the gas shell, and it permits the analysis of all the molecules using a homogeneus criteria. Also, the size of the emitting region for each molecule has to be known to account for the proper dilution of the emission. We have adopted approximate sizes of the emitting regions of $\theta_{\mathrm{s}}\left({ }^{12} \mathrm{CO},{ }^{13} \mathrm{CO}\right)=18^{\prime \prime}$ which is a representative value for the sizes measured in Bujarrabal \& Alcolea (1991), Castro-Carrizo et al. (2010), and Kim et al. (2010). We also adopted the sizes $\theta_{\mathrm{s}}\left(\mathrm{HCN}, \mathrm{H}^{13} \mathrm{CN}\right)=4^{\prime \prime}\left(\right.$ Marvel 2005), $\theta_{\mathrm{s}}=2^{\prime \prime}$ for $\mathrm{SiO}$ isotopologues (Lucas et al. 1992), and $\theta_{\mathrm{s}} \lesssim 0$ "' 7 for PO and PN (De Beck et al. 2013). Similar values have been also used for these molecules by Kim et al. (2010). For the rest of the molecules, which have not been mapped, we assumed emitting 
region sizes according to observational constraints, previous estimations, or predictions by radiative transfer and chemical models. These values are given in Table 5 and discussed in Sect. 6. We note that the adopted sizes are uncertain for some molecules and also that the emitting region size may vary for each transition of a given molecule. In general, an underestimation of the size of any emitting region will cause an underestimation of the beam filling factor (see Eq. (3)) and, thus, an overestimation of the abundance, and vice versa.

There are additional sources of uncertainty in the values derived from the population diagrams. In particular, the kinetic temperature $\left(T_{\text {kin }}\right)$ throughout the CSE is not expected to be constant. This effect is less important for the molecules whose emission arises from regions with very uniform physical conditions (inner layers, photodissociation layers, etc.), and it must be more important for species whose emission extends through the whole envelope, such as probably $\mathrm{SO}_{2}$, for which we did a more detailed radiative transfer analysis (see Sect. 4.3).

In the population diagrams, we have included lines (unambigously identified and unblended) with fluxes above $5 \sigma$. For those molecules well known to be present in the envelope of IK Tau, we have also included lines with 3-5 $\sigma$ detections. In the case of molecules with a hyperfine structure that cannot be spectrally resolved with the spectral resolution achieved, that is, $\mathrm{CN}$, $\mathrm{NS}$, and NO, we measured the total integrated intensity of the blend of the hyperfine components and we calculated the sum of the strength of the hyperfine components to compute their population diagrams. In the case of $\mathrm{H}_{2} \mathrm{~S}$ we fitted together the lines of the ortho and para species due to the scarce number of lines detected for each species individually.

We have not calculated population diagrams for the molecules $\mathrm{SiO} v=1, \mathrm{SiO} v=2, \mathrm{SiO} v=3,{ }^{29} \mathrm{SiO} v=1$, ${ }^{29} \mathrm{SiO} v=2,{ }^{29} \mathrm{SiO} v=3,{ }^{30} \mathrm{SiO} v=1,{ }^{30} \mathrm{SiO} v=2, \mathrm{H}_{2} \mathrm{O}$, $\mathrm{H}_{2} \mathrm{O} v_{2}=1$ or $\mathrm{H}_{2} \mathrm{O} v_{2}=2$. Some of these lines are masers and their populations are expected to strongly deviate from a Boltzmann distribution. Also, the intensity of the lines of these molecules may vary with the stellar pulsation phase and, given that the observations spread over a period of time of approximately five years, the excitation conditions may have changed along the observational runs (see Sect. 6.1).

The fractional abundances averaged in the emitting region for the molecules detected are calculated using the following equation:

$f(X)=N(X) \frac{f\left({ }^{13} \mathrm{CO}\right)}{N\left({ }^{13} \mathrm{CO}\right)}$,

where $f(\mathrm{X})$ and $N(\mathrm{X})$ represent the fractional abundance (with respect to $\mathrm{H}_{2}$ ) and column density of the molecule analysed, respectively. $N\left({ }^{13} \mathrm{CO}\right)$ is the column density that we derived from the ${ }^{13} \mathrm{CO}$ population diagram, and $f\left({ }^{13} \mathrm{CO}\right)=1.4 \times 10^{-5}$ (Decin et al. 2010a).

\subsection{Radiative transfer model: MADEX}

MADEX (Cernicharo 2012) is a radiative transfer code which is able to operate under LTE and large velocity gradient (LVG) approximation (Goldreich \& Kwan 1974). It solves the radiative transfer problem coupled with statistical equilibrium equations to derive the radiation field and populations of the levels on each point of a gas cloud. Then, the emergent profile of the lines is obtained through ray-tracing.

We used MADEX to calculate line opacities, excitation temperatures, and critical densities for the physical conditions expected at a given distance from the star, that is, $n(r)$ and $T_{\text {kin }}(r)$
Table 4. Parameters of the central Mira-type star and the CSE of IK Tau.

\begin{tabular}{lcc}
\hline \hline Parameter & Value & Reference \\
\hline Distance $(d)$ & $265 \mathrm{pc}$ & $\mathrm{a}$ \\
Stellar radius $\left(R_{*}\right)$ & $2.5 \times 10^{13} \mathrm{~cm}$ & $\mathrm{c}$ \\
Stellar effective temperature $\left(T_{*}\right)$ & $2200 \mathrm{~K}$ & $\mathrm{c}$ \\
Terminal expansion velocity $\left(v_{\infty}\right)$ & $18.5 \mathrm{~km} \mathrm{~s}^{-1}$ & $\mathrm{e}$ \\
AGB mass loss rate $(\dot{M})$ & $8 \times 10^{-6} M_{\odot} \mathrm{yr}^{-1}$ & $\mathrm{c}$ \\
Gas kinetic temperature $\left(T_{\text {kin }}\right)$ & $T_{*}\left(r / R_{*}\right)^{-0.6}$ & $\mathrm{c}$ \\
Dust temperature $\left(T_{\mathrm{d}}\right)$ & $T_{*}\left(2 r / R_{*}\right)^{-0.4}$ & $\mathrm{~b}$ \\
Dust condensation radius $\left(R_{\mathrm{c}}\right)$ & $8 R_{*}$ & $\mathrm{~d}$ \\
Density of particles $(n)$ & $\dot{M} /\left(4 \pi r^{2}\left\langle m_{\mathrm{g}}\right\rangle v_{\exp }\right)$ & - \\
\hline
\end{tabular}

Notes. The density of particles is calculated with the law of conservation of mass, where $\left\langle m_{\mathrm{g}}\right\rangle$ is the mean mass of gas particles (2.3 amu, after considering $\mathrm{H}_{2}, \mathrm{He}$, and CO). (a): Hale et al. (1997); (b): Decin et al. (2006); (c): Decin et al. (2010a); (d): Gobrecht et al. (2016); (e): this work (see Sect. 5.1).

(see Table 4), along with the column densities derived from the population diagrams (given in Table 5), and the $v_{\text {exp }}$ derived from the line fitting (given in Table A.1) for all the molecules detected. We systematically used this procedure to compare the line opacities obtained from the interpretation of the line profiles and to verify if a given molecule could be sub-thermally excited or not. These calculations are detailed in each subsection of the Sect 6. The sets of the collisional coefficients used for these calculations (when available) are: CO (Yang et al. 2010), $\mathrm{SiO}$ (Dayou \& Balança 2006), $\mathrm{H}_{2} \mathrm{O}$ (Daniel et al. 2011), HCN (Ben Abdallah et al. 2012), CS (Lique \& Spielfiedel 2007), SiS (Toboła et al. 2008), NaCl (Quintana-Lacaci et al. 2016), SO (Lique et al. 2007), PN (Toboła et al. 2007), CN (Lique \& Kłos 2011), $\mathrm{HCO}^{+}$(Flower 1999), and $\mathrm{H}_{2} \mathrm{CO}$ (Schöier et al. 2005, and references therein). We also obtained synthetic spectra that helped us to correctly identify the spectral features.

As it is shown in Sect 5.2, the detected $\mathrm{SO}_{2}$ transitions have a large span in energies (i.e. from 7.7 up to $733.4 \mathrm{~K}$ ). The population diagram analysis of this molecule indicates that it is possibly tracing out an inner region of the CSE, with a $T_{\text {kin }} \gtrsim 290 \mathrm{~K}$ which is not observed for other molecules, and also a more external region with $T_{\text {rot }} \sim 40 \mathrm{~K}$ from where most of the emission of the rest of the molecules arises. To investigate more precisely the excitation conditions of $\mathrm{SO}_{2}$ and to confirm or not the temperature stratification inferred from the population diagrams, we have performed a detailed radiative transfer calculation for $\mathrm{SO}_{2}$. Our main goal is to investigate the presence of $\mathrm{SO}_{2}$ in the innermost regions of the CSE.

The physical model consists on a spherical expanding envelope of dust and gas with a constant mass loss rate, similar to the model presented in Agúndez et al. (2012) but corrected for IK Tau (see Table 4). The radius of the star is $2.5 \times 10^{13} \mathrm{~cm}$ (Decin et al. 2010a). The density of particles is calculated with the law of conservation of mass, that is, $n \propto r^{-2}$ (valid for a CSE expanding at constant velocity). In terms of $v_{\text {exp }}$, the envelope is divided in three regions: $(i)$ from 1 to $5 R_{*}$ where $v_{\exp }=5 \mathrm{~km} \mathrm{~s}^{-1}$ as an average value in this region (Decin et al. 2010b), (ii) from 5 to $8 R_{*}$ where $v_{\text {exp }}=10 \mathrm{~km} \mathrm{~s}^{-1}$ according to the dust condensation radius (Gobrecht et al. 2016), and (iii) from $8 R_{*}$ to the end of the CSE where $v_{\exp }=18.5 \mathrm{~km} \mathrm{~s}^{-1}$ (see Sect.5.1). Concerning dust, we adopted a dust-to-gas ratio of $1.3 \times 10^{-4}$ for the region $(i)$, a value of $1.2 \times 10^{-3}$ for region $(\mathrm{ii})$ and a value of $2.3 \times 10^{-3}$ for the region (iii), according to the values given by Gobrecht et al. (2016) for silicates. The dust temperature has been taken from Decin et al. (2006; see Table 4). The optical 
Table 5. Results from the population diagrams, sorted according to the molecular fractional abundance (relative to $\mathrm{H}_{2}$ ) in descending order.

\begin{tabular}{|c|c|c|c|c|c|c|c|c|}
\hline Molecule & $\begin{array}{l}T_{\text {rot }} \\
(\mathrm{K})\end{array}$ & $\begin{array}{l}\text { Column density } \\
\left(\mathrm{cm}^{-2}\right)\end{array}$ & $\begin{array}{l}\text { Abundance } \\
-\end{array}$ & $\begin{array}{l}\theta_{\mathrm{s}} \\
\prime \prime\end{array}$ & $\begin{array}{l}\text { Lit. observational } f(\mathrm{X}) \\
-\end{array}$ & $\begin{array}{l}\text { Ref. } \\
-\end{array}$ & $\begin{array}{l}\text { Lit. chemical model } f(\mathrm{X}) \\
-\end{array}$ & $\begin{array}{l}\text { Ref. } \\
-\end{array}$ \\
\hline $\mathrm{CO}$ & $18(4)$ & $\gtrsim 1.3(0.3) \times 10^{17}$ & $\gtrsim 1.1 \times 10^{-4}$ & 18 & {$[2-3] \times 10^{-4}$} & $\mathrm{~d}, \mathrm{f}$ & $7 \times 10^{-4}-1 \times 10^{-3}$ & $\gamma$ \\
\hline${ }^{13} \mathrm{CO}$ & $21(8)$ & $1.6(0.6) \times 10^{16}$ & $1.4 \times 10^{-5 \dagger}$ & 18 & {$[1-3] \times 10^{-5}$} & $\mathrm{~d}, \mathrm{f}$ & - & - \\
\hline $\mathrm{SO}_{2}$ cold & $40(2)$ & $>1.1(0.1) \times 10^{16}$ & $>9.6 \times 10^{-6}$ & $2^{\ddagger}$ & $2 \times 10^{-6}-1 \times 10^{-5}$ & $b, f$ & $2 \times 10^{-7}$ & $\epsilon$ \\
\hline $\mathrm{SiO}$ & $17(2)$ & $>9.1(3.3) \times 10^{15}$ & $>8.0 \times 10^{-6}$ & 2 & $4 \times 10^{-7}-2 \times 10^{-5}$ & $e, d$ & $2 \times 10^{-5}-9 \times 10^{-5}$ & $\gamma$ \\
\hline SO & $17(1)$ & $\gtrsim 8.9(1.6) \times 10^{15}$ & $\gtrsim 7.8 \times 10^{-6}$ & 2 & $3 \times 10^{-7}-3 \times 10^{-6}$ & $\mathrm{f}, \mathrm{a}$ & $2 \times 10^{-12}-9 \times 10^{-7}$ & $\gamma, \epsilon$ \\
\hline $\mathrm{SiS}$ & $64(5)$ & $5.2(0.6) \times 10^{15}$ & $4.6 \times 10^{-6}$ & 2 & $8 \times 10^{-9}-1 \times 10^{-5}$ & $\mathrm{~d}$ & $8 \times 10^{-10}-3 \times 10^{-6}$ & $\beta, \epsilon$ \\
\hline $\mathrm{SO}_{2}$ warm & $290(60)$ & $>3.1(1.0) \times 10^{15}$ & $>2.7 \times 10^{-6 \ddagger}$ & $2^{\ddagger}$ & - & - & $2 \times 10^{-14}-4 \times 10^{-9}$ & $\gamma$ \\
\hline${ }^{29} \mathrm{SiO}$ & $17(2)$ & $\gtrsim 2.1(0.6) \times 10^{15}$ & $\gtrsim 1.8 \times 10^{-6}$ & 2 & - & - & - & - \\
\hline PO & $17(2)$ & $2.0(0.4) \times 10^{15}$ & $1.7 \times 10^{-6}$ & 0.7 & $5 \times 10^{-8}-6 \times 10^{-7}$ & $\mathrm{c}$ & $2 \times 10^{-10}-1 \times 10^{-7}$ & $\gamma$ \\
\hline${ }^{30} \mathrm{SiO}$ & $17(2)$ & $\gtrsim 1.5(0.5) \times 10^{15}$ & $\gtrsim 1.3 \times 10^{-6}$ & 2 & - & - & - & - \\
\hline $\mathrm{H}_{2} \mathrm{~S}$ & $39(3)$ & $1.4(0.3) \times 10^{15}$ & $1.2 \times 10^{-6}$ & 2 & - & - & $6 \times 10^{-13}-3 \times 10^{-5}$ & $\beta, \gamma$ \\
\hline${ }^{34} \mathrm{SO}_{2}$ & $35(6)$ & $1.1(0.3) \times 10^{15}$ & $9.6 \times 10^{-7}$ & 2 & - & - & $\begin{array}{l}- \\
0 \times 10 \\
0\end{array}$ & $\begin{array}{l}\rho, \gamma \\
-\end{array}$ \\
\hline $\mathrm{CS}$ & $25(2)$ & $9.2(1.0) \times 10^{14}$ & $8.0 \times 10^{-7}$ & 2 & $8 \times 10^{-8}-3 \times 10^{-7}$ & $f, g$ & $2 \times 10^{-11}-2 \times 10^{-5}$ & $\gamma, \beta$ \\
\hline${ }^{34} \mathrm{SO}$ & $14(1)$ & $8.9(2.4) \times 10^{14}$ & $7.8 \times 10^{-7}$ & 2 & $\begin{array}{l}0 \\
-\end{array}$ & - & $\begin{array}{l}- \\
-10\end{array}$ & $\begin{array}{l}\gamma, \beta \\
-\end{array}$ \\
\hline PN & $18(2)$ & $8.3(1.7) \times 10^{14}$ & $7.3 \times 10^{-7}$ & 0.7 & $3 \times 10^{-7}$ & $\mathrm{c}$ & $4 \times 10^{-10}-6 \times 10^{-7}$ & $\gamma$ \\
\hline $\mathrm{HCN}$ & $10(1)$ & $>7.6(2.9) \times 10^{14}$ & $>6.6 \times 10^{-7}$ & 4 & $4 \times 10^{-7}-1 \times 10^{-6}$ & i, f & $6 \times 10^{-12}-3 \times 10^{-4}$ & $\gamma, \beta$ \\
\hline${ }^{29} \mathrm{SiS}$ & $78(5)$ & $4.9(0.4) \times 10^{14}$ & $4.3 \times 10^{-7}$ & 2 & - & - & - & - \\
\hline $\mathrm{Si}^{34} \mathrm{~S}$ & $70(10)$ & $4.5(0.8) \times 10^{14}$ & $3.9 \times 10^{-7}$ & 2 & - & - & - & - \\
\hline $\mathrm{NaCl}$ & $67(7)$ & $3.5(0.6) \times 10^{14}$ & $3.1 \times 10^{-7}$ & 0.3 & $4 \times 10^{-9}$ & $\mathrm{~h}$ & $4 \times 10^{-12}-1 \times 10^{-8}$ & $\gamma$ \\
\hline${ }^{30} \mathrm{SiS}$ & $83(9)$ & $3.5(0.5) \times 10^{14}$ & $3.1 \times 10^{-7}$ & 2 & - & - & - & - \\
\hline $\mathrm{H}_{2} \mathrm{CO}$ & $10(2)$ & $2.8(0.5) \times 10^{14}$ & $2.4 \times 10^{-7}$ & 2 & - & - & $1 \times 10^{-9}-5 \times 10^{-7}$ & $\alpha, \epsilon$ \\
\hline $\mathrm{CN}$ & $9(2)$ & $1.5(0.7) \times 10^{14}$ & $1.3 \times 10^{-7}$ & 6 & $2 \times 10^{-10}-2 \times 10^{-7}$ & $\mathrm{~d}, \mathrm{f}$ & $3 \times 10^{-13}-3 \times 10^{-7}$ & $\beta, \delta$ \\
\hline $\mathrm{Si}^{18} \mathrm{O}$ & $24(8)$ & $1.5(0.8) \times 10^{14}$ & $1.3 \times 10^{-7}$ & 2 & - & - & - & - \\
\hline $\mathrm{Si}^{17} \mathrm{O}$ & $12(2)$ & $1.0(0.4) \times 10^{14}$ & $8.7 \times 10^{-8}$ & 2 & - & - & - & - \\
\hline${ }^{13} \mathrm{CS}$ & $34(3)$ & $9.6(0.8) \times 10^{13}$ & $8.4 \times 10^{-8}$ & 2 & - & - & - & - \\
\hline $\mathrm{H}^{13} \mathrm{CN}$ & $11(2)$ & $9.2(2.5) \times 10^{13}$ & $8.0 \times 10^{-8}$ & 4 & - & - & - & - \\
\hline $\mathrm{C}^{34} \mathrm{~S}$ & $24(1)$ & $6.9(0.6) \times 10^{13}$ & $6.0 \times 10^{-8}$ & 2 & - & - & - & - \\
\hline NS & $18(2)$ & $2.6(0.5) \times 10^{13}$ & $2.3 \times 10^{-8}$ & 6 & - & - & $7 \times 10^{-13}-8 \times 10^{-9}$ & $\beta, \delta$ \\
\hline
\end{tabular}

Notes. We give the formal uncertainties derived from the population diagram fits within parentheses. Column 5: size adopted for the emitting region. Columns 6 and 7: range of the fractional abundances given in the literature, derived from the following works based on observations: (a): Bujarrabal et al. (1994); (b): Danilovich et al. (2016); (c): De Beck et al. (2013); (d): Decin et al. (2010a); (e): González Delgado et al. (2003); (f): Kim et al. (2010); (g): Lindqvist et al. (1988): (h): Milam et al. (2007); (i): Schöier et al. (2013). Columns 8 and 9: range of the fractional abundances given in the literature, which were derived from the following chemical and shocks models: $(\alpha)$ : Agúndez et al. (2010); $(\beta)$ : Duari et al. (1999); $(\gamma)$ : Gobrecht et al. (2016); $(\delta)$ : Li et al. (2016); $(\epsilon)$ : Willacy \& Millar (1997). ${ }^{(\dagger)}$ Value given in Table 6 in Decin et al. (2010a). $\left.{ }^{\ddagger}\right)$ See Sect. 6.5.1.

properties of the silicate dust and the size of the grains $(0.1 \mu \mathrm{m})$ have been adopted from Suh (1999). For the microturbulence velocity we adopted the values given in Agúndez et al. (2012) for the C-rich CSE IRC+10216. We used different $\mathrm{SO}_{2}$ abundance profiles (see Fig. 5) in order to investigate the presence of warm $\mathrm{SO}_{2}$ in the inner regions of the CSE. For the calculations we used a set of collisional coefficients which are described in Appendix E. The results of the radiative transfer model are shown in Fig. 11 and discussed in Sect. 6.5.1.

\section{Results}

Here we present the general results from the analysis, which are summarised in Table 5 and Fig. 6.

\subsection{Expansion velocity}

The terminal expansion velocity of the CSE $\left(v_{\infty}\right)$ can be estimated from the linewidths of the spectral features that arise from the outer ( $r>8 R_{*}$, Gobrecht et al. 2016) envelope regions where the gas has been fully accelerated to this maximal velocity. We estimated the $v_{\infty}$ from the ${ }^{13} \mathrm{CO}$ linewidths (see Table A.1) given that ${ }^{13} \mathrm{CO}$ emission certainly extends beyond the wind acceleration region, and the line profiles display a clear U-shaped profile (see Fig. 3) and, thus, no significant

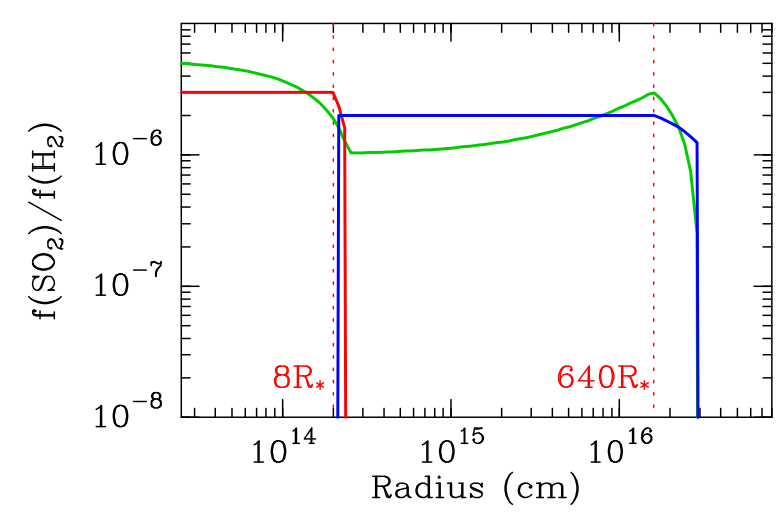

Fig. 5. Radial profiles of the abundance adopted for the radiative transfer model of the lines of $\mathrm{SO}_{2}$. We tested three different radial profiles: (i) an inner distribution of $\mathrm{SO}_{2}$ with $f\left(\mathrm{SO}_{2}\right)=3 \times 10^{-6}$ between $1-8 R_{*}$ (red line); (ii) a constant radial profile with $f\left(\mathrm{SO}_{2}\right)=2 \times 10^{-6}$ between 8 $640 R_{*}$ (blue line); and (iii) a two component profile (green line) which includes an inner warm component plus an extended component of $\mathrm{SO}_{2}$. The results of the different models are shown in Fig. 11.

opacity broadening is expected (Phillips et al. 1979). Assuming a mean density of $n\left(\mathrm{H}_{2}\right)=10^{5} \mathrm{~cm}^{-3}$, and the temperature and column density given in Table 5 , the highest opacity measured 

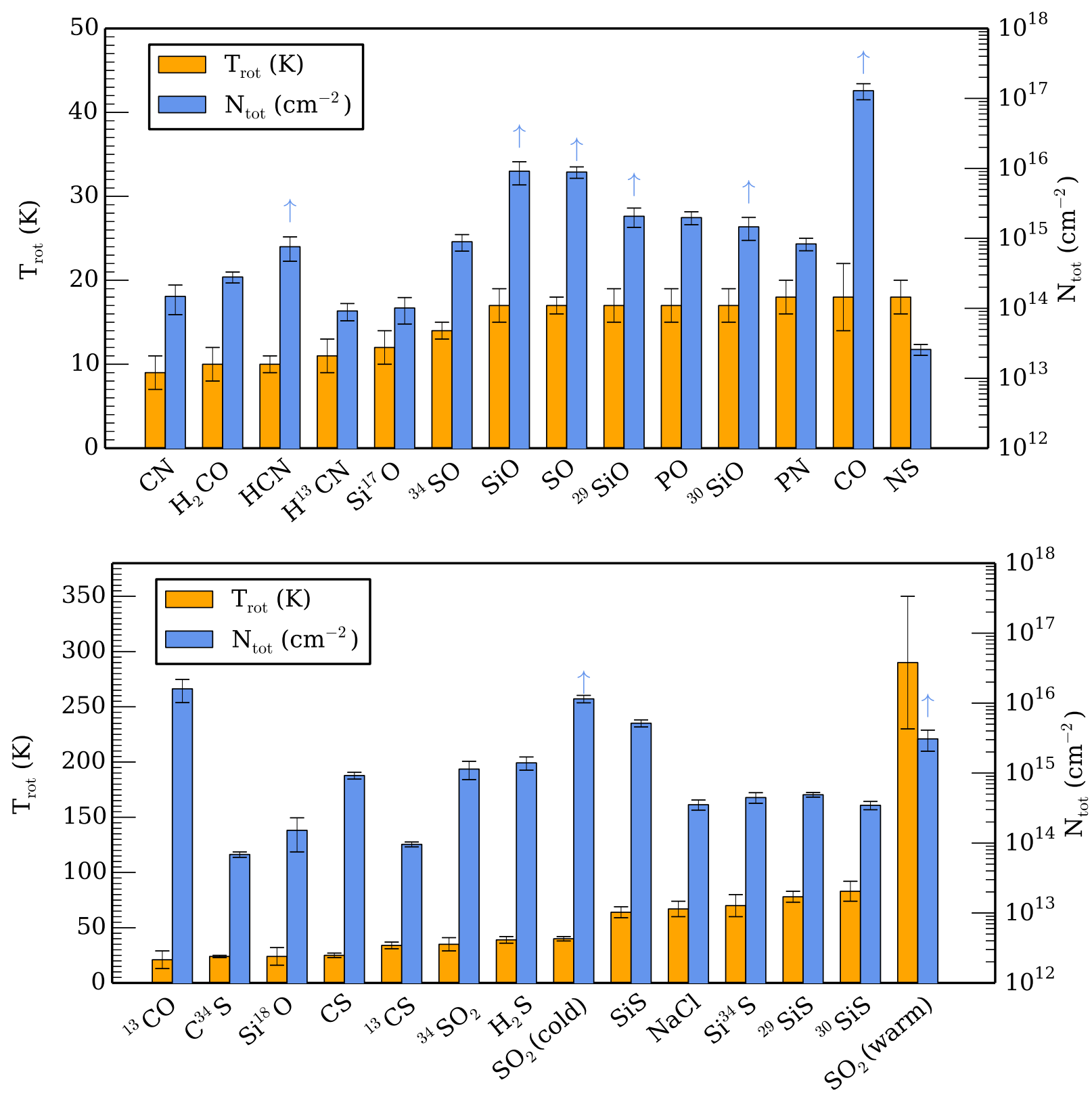

Fig. 6. Graph of the rotational temperatures (orange bars) and column densities (blue bars), with their formal uncertainties (black lines), derived from the population diagrams. Vertical blue arrows are plotted to indicate column density lower limits over each corresponding molecule. The temperature scale is represented on the left vertical axis, and the column density logarithmic scale is represented on the right vertical axis. The limits of the temperature scale are different in both boxes to improve the visual aspect of the figures. The values represented are tabulated in Table 5 .

with MADEX is $\tau=0.13$ for the ${ }^{13} \mathrm{CO} J=3-2$ line. We derived $v_{\infty}=18.6 \pm 1.2 \mathrm{~km} \mathrm{~s}^{-1}$ which is in good agreement with previous measurements (e.g. De Beck et al. 2013).

Most of the rest of the lines detected display linewidths consistent with $v_{\infty}$ like for example SiS (see Tables A.1, A.2 and Fig. 7). In the particular case of $\mathrm{H}_{2} \mathrm{CO}$, we stacked the lines with $K=0, K=1$, and $K=2$ to confirm that $\mathrm{H}_{2} \mathrm{CO}$ linewidths are consistent with $v_{\text {exp }}=v_{\infty}$ (Fig. 8). There are also several lines with linewidths larger than $v_{\infty}$, due to a blend of several hyperfine components (as occurs for NO and NS), or a poor fitting for lines detected below $5 \sigma$.

Additionally, the lines with $E_{\mathrm{u}} \gtrsim 160 \mathrm{~K}$, that is, the lines of vibrationally excited states (Table A.2), $\mathrm{H}_{2} \mathrm{O}, \mathrm{PO}, \mathrm{NaCl}$, and several high- $E_{\mathrm{u}} \mathrm{SO}_{2}$ lines, have line profiles indicative of $v_{\text {exp }} \lesssim 10 \mathrm{~km} \mathrm{~s}^{-1}$, consistent with emission from the inner regions of the CSE where the gas is still being accelerated $\left(r<8 R_{*}\right.$, i.e. $\sim 2 \times 10^{14} \mathrm{~cm}^{-2}$, Decin et al. 2010b; Gobrecht et al. 2016). In order to obtain a more reliable estimate of the $\mathrm{NaCl}$ linewidths, we stacked the lines with $E_{\mathrm{u}}=[22.5-56.9] \mathrm{K}$ (group 1), $E_{\mathrm{u}}=[85.0-118.7] \mathrm{K}$ (group 2), and $E_{\mathrm{u}}=[131.2-187.4] \mathrm{K}$ (group 3) (see Fig. 8). The linewidths measured are consistent with $v_{\exp }=14.8 \pm 2.2 \mathrm{~km} \mathrm{~s}^{-1}\left(\right.$ group 1), $v_{\exp }=20.1 \pm 1.1 \mathrm{~km} \mathrm{~s}^{-1}$ (group 2), and $v_{\exp }=11.8 \pm 1.1 \mathrm{~km} \mathrm{~s}^{-1}$ (group 3). Therefore, it seems that $v_{\exp }<v_{\infty}$ at least for the lines of the group 3 , that is to say, the high- $E_{\mathrm{u}}$ lines.

There are also some lines with low $E_{\mathrm{u}}(<200 \mathrm{~K})$ which appear to have expansion velocities of $\sim 10 \mathrm{~km} \mathrm{~s}^{-1}$ (e.g. $\mathrm{SO}_{2}$, see Fig. 7). Such low values of the $v_{\exp }$ were measured for weak $\mathrm{SO}_{2}$ lines with low $\mathrm{S} / \mathrm{N}$, thus, the linewidths observed in their profiles are uncertain. 
L. Velilla Prieto et al.: The millimeter IRAM-30 m line survey toward IK Tauri
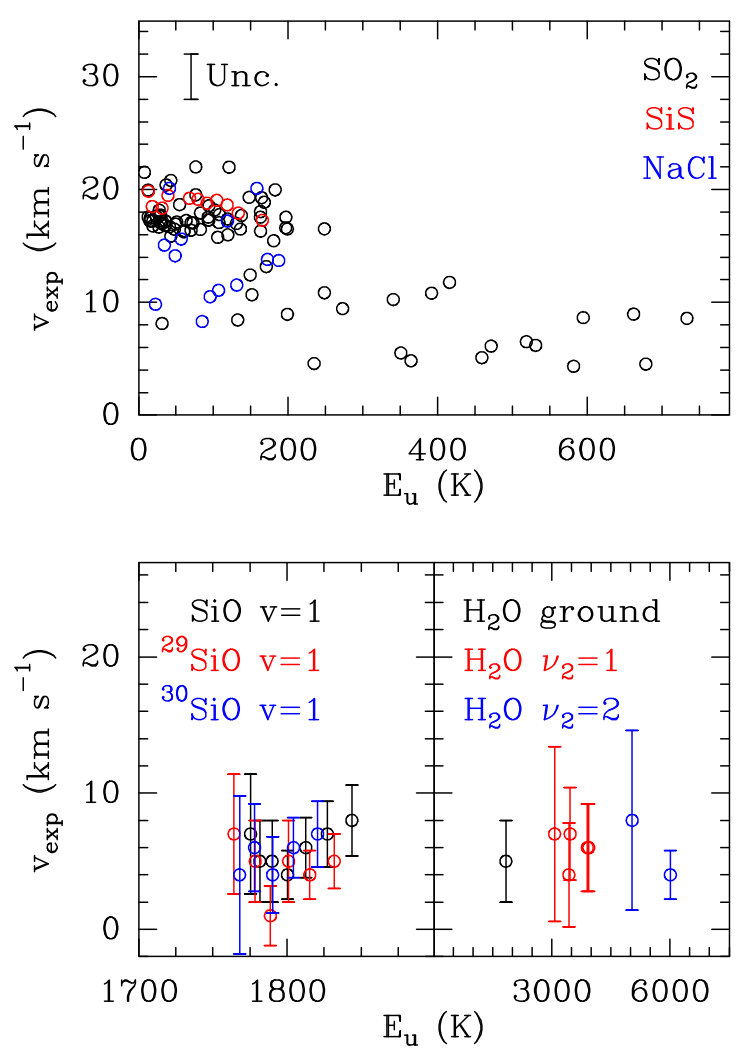

Fig. 7. Plot of the $v_{\exp }$ derived from the fit of the lines as a function of the $E_{\mathrm{u}}$ of the corresponding transition for several molecules. In the top, we did not represent each error bar to improve the visualization of the figure. A typical error bar of $4 \mathrm{~km} \mathrm{~s}^{-1}$ is plotted in the top left corner of the box. The values and uncertainties of the bottom correspond to the values given in Table A.2. For those lines that were observed in different epochs, we adopted an average value of the different measurements for the $v_{\exp }$ and its uncertainty.

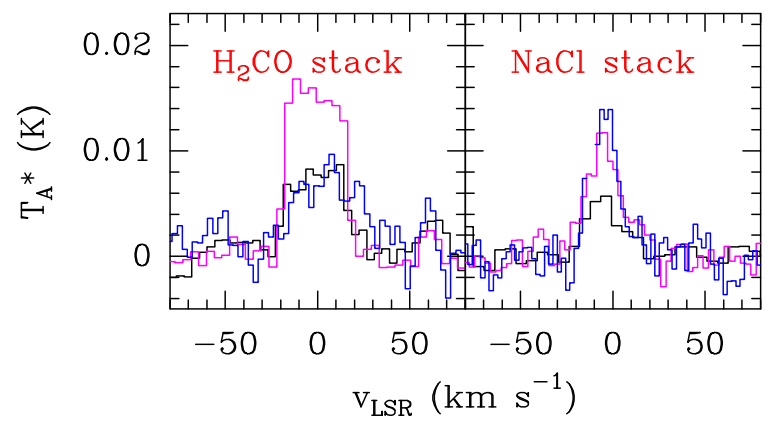

Fig. 8. Line stacking of the $\mathrm{H}_{2} \mathrm{CO}$ (left) and the $\mathrm{NaCl}$ (right) lines detected, with a spectral resolution of $2 \mathrm{MHz}$. Black spectrum correspond to the $\mathrm{H}_{2} \mathrm{CO} K_{\mathrm{a}}=0$ lines and the $\mathrm{NaCl}$ lines with $E_{\mathrm{u}}=[22.5-56.9] \mathrm{K}$, pink spectrum correspond to the $\mathrm{H}_{2} \mathrm{CO} K_{\mathrm{a}}=1$ lines and the $\mathrm{NaCl}$ lines with $E_{\mathrm{u}}=[85.0-118.7] \mathrm{K}$, and blue spectrum correspond to the $\mathrm{H}_{2} \mathrm{CO}$ $K_{\mathrm{a}}=2$ lines and the $\mathrm{NaCl}$ lines with $E_{\mathrm{u}}=[131.2-187.4] \mathrm{K}$.

\subsection{Rotational temperatures, column densities and fractional abundances}

The population diagrams for all the molecules detected are shown in Appendix D, in Figs. D.1-D.13. Most diagrams display a linear trend, however, the population diagrams of, for example, $\mathrm{SiO},{ }^{29} \mathrm{SiO}$, or $\mathrm{SO}$, display departures from a linear behaviour, which are more notable for low $J$ transitions (see e.g. Fig. D.2). These departures are discussed in detail in Sect. 6
Table 6. Isotopic ratios obtained from the abundances derived with the population diagrams.

\begin{tabular}{lll}
\hline \hline Ratio & Value & From \\
\hline${ }^{12} \mathrm{C} /{ }^{13} \mathrm{C}$ & $>8(\sim 10)$ & $\mathrm{CO}$ \\
${ }^{12} \mathrm{C} /{ }^{13} \mathrm{C}$ & 10 & $\mathrm{CS}$ \\
${ }^{12} \mathrm{C} /{ }^{13} \mathrm{C}$ & $>8(\sim 15)$ & $\mathrm{HCN}$ \\
${ }^{16} \mathrm{O} /{ }^{18} \mathrm{O}$ & $\gg 61$ & $\mathrm{SiO}$ \\
${ }^{16} \mathrm{O} /{ }^{17} \mathrm{O}$ & $\gg 91$ & $\mathrm{SiO}$ \\
${ }^{28} \mathrm{Si} /{ }^{29} \mathrm{Si}$ & $>4(\sim 18)$ & $\mathrm{SiO}$ \\
${ }^{28} \mathrm{Si} /{ }^{29} \mathrm{Si}$ & 11 & $\mathrm{SiS}$ \\
${ }^{28} \mathrm{Si} /{ }^{30} \mathrm{Si}$ & $>6(\sim 34)$ & $\mathrm{SiO}$ \\
${ }^{28} \mathrm{Si} /{ }^{30} \mathrm{Si}$ & 16 & $\mathrm{SiS}$ \\
${ }^{32} \mathrm{~S} /{ }^{34} \mathrm{~S}$ & $>8(\sim 15)$ & $\mathrm{SO}$ \\
${ }^{32} \mathrm{~S} /{ }^{44} \mathrm{~S}$ & 13 & $\mathrm{CS}$ \\
${ }^{32} \mathrm{~S} /{ }^{34} \mathrm{~S}$ & 12 & $\mathrm{SiS}$ \\
${ }^{32} \mathrm{~S} /{ }^{34} \mathrm{~S}$ & $>10(\sim 13) \dagger$ & $\mathrm{SO}_{2}$ \\
\hline
\end{tabular}

Notes. Opacity corrected values are given between parentheses (see Sect.6.10). ${ }^{(\dagger)}$ This value was computed using only the cold $\mathrm{SO}_{2}$ component in Table 5 .

and they reflect the effect of optically thick emission and/or subthermal excitation. For $\mathrm{SO}_{2}$ we see two different trends for lines below and above $E_{\mathrm{u}}=160 \mathrm{~K}$. We fitted both separately and their implications are explained in Sect. 6.5.1.

The rotational temperatures derived range from $9 \mathrm{~K}$ (for $\mathrm{CN}$ ) to $290 \mathrm{~K}$ (for the warm component of $\mathrm{SO}_{2}$ ), with most of the molecules displaying rotational temperatures between $15 \mathrm{~K}$ and $40 \mathrm{~K}$ (see Fig. 6). The column densities range from $\gtrsim 1.3 \times 10^{17} \mathrm{~cm}^{-2}$ for ${ }^{12} \mathrm{CO}$, down to $2.6 \times 10^{13} \mathrm{~cm}^{-2}$ for NS. We obtained averaged fractional abundances using the Eq. (9), which range between $>1.1 \times 10^{-4}$ for ${ }^{12} \mathrm{CO}$, down to $2.3 \times 10^{-8}$ for NS. We calculated the isotopic ratios of the molecules for which several isotopologues were detected. Results are presented in Table 6 and discussed in Sect. 6.10.

\section{Discussion: an overall picture of the whole envelope}

\subsection{Variability}

The excitation mechanisms of the lines are a mix of collisional and radiative procceses, where the radiation emitted by the central star has an impact on the population of the rotational levels (e.g. $\mathrm{H}_{2} \mathrm{O}$ Agúndez \& Cernicharo 2006). Since AGB stars are variable with periods of one to two years, the net excitation mechanism for those molecules is variable and the results obtained from the analysis of the molecular lines of AGB CSEs could be affected by this variability. In the case of O-rich stars, the impact of radiative pumping effects on masers is well-known (e.g. Nakashima \& Deguchi 2007). Thermal line observations of CSEs have also to be considered carefully in the sub-millimeter and far-IR domain, while for the millimeter wavelength range, specially for low- $J$ lines, the variation of the stellar light has not a major impact (Cernicharo et al. 2014). Given that our observations spread over a period of approximately five years, we observed a few spectral ranges at different epochs and here we discuss the results observed.

For the spectra observed at different epochs we observed intensity variations of less than $25 \%$ for the rotational lines of the 


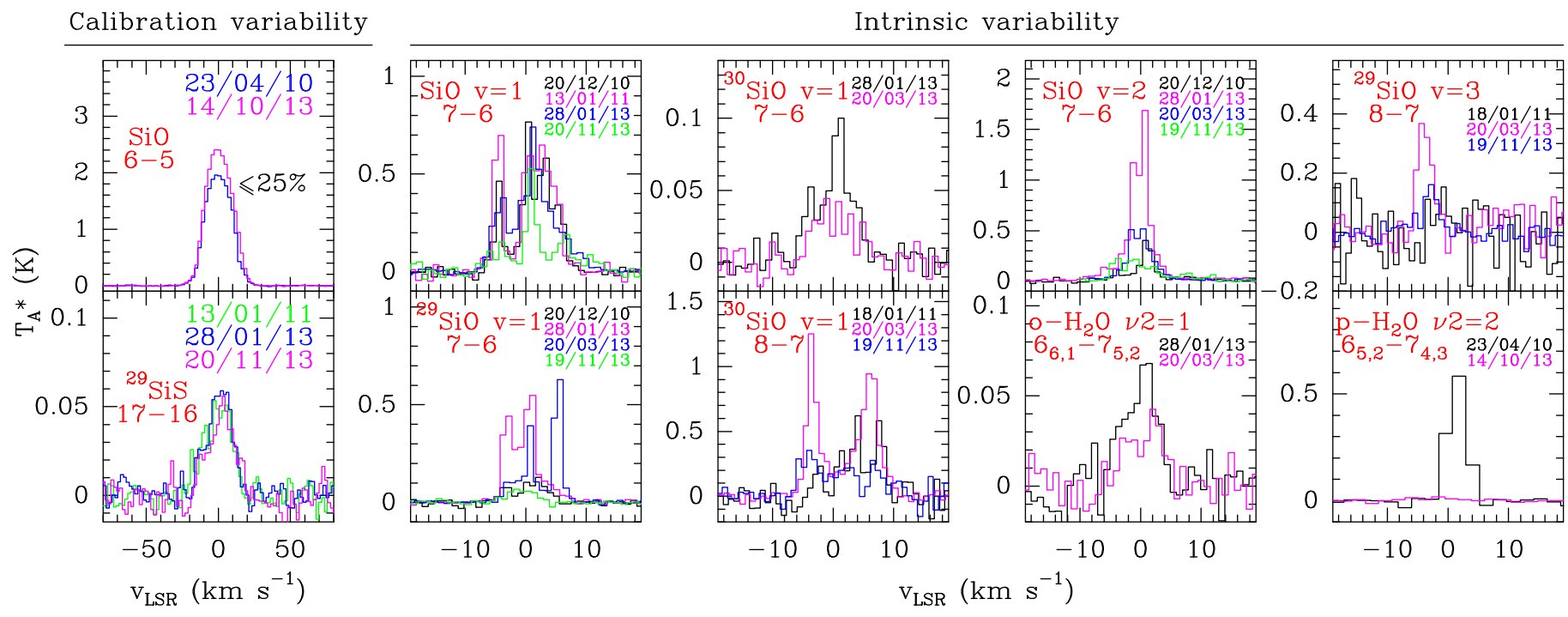

Fig. 9. Variability of some of the lines observed. The observation dates which correspond to the spectra are shown in the top right corner of each box with its corresponding colour. The spectral resolution is $2 \mathrm{MHz}$. As discussed in Sect. 6.1, variations of up to a $25 \%$ are within the uncertainties of the calibration, pointing and baseline substraction (i.e. left boxes). Variations of intensity higher than a $25 \%$ are observed in vibrationally excited lines or masers (i.e. right boxes).

ground vibrational levels. This value (i.e. $25 \%$ ) was taken as the calibration uncertainty (see Sect. 2), and considered in the results presented throughout this work (Fig. 9 left). However, we observed strong intensity variations $(>25 \%)$ for several lines, like for example the $\mathrm{SiO} v=1 J=7-6$, (see Table A.2 and Fig. 9). These strongly variable lines correspond to rotational transitions of vibrationally excited states with $v_{\text {exp }} \lesssim 10 \mathrm{~km} \mathrm{~s}^{-1}$, which arise from very inner regions of the CSE. The excitation mechanism of these lines may be correlated with the stellar phase, thus, it is not possible to extract any information of their abundances. In Table A.2 we present the fit parameters for these vibrationally excited and maser lines, where we included the mean Julian date of the observation and, in case that the feature was observed in different epochs, multiple measurements of that spectral feature.

\subsection{O-bearing molecules}

$\mathrm{CO}$ is the most abundant molecule in IK Tau (after $\mathrm{H}_{2}$ ). It is distributed along the whole CSE with a size of $\sim[7-8] \times 10^{16} \mathrm{~cm}$ (see Sect. 1.1). The ${ }^{12} \mathrm{CO} J=2-1$ and ${ }^{12} \mathrm{CO} J=3-2$ lines display parabolic profiles typical of optically thick lines, while the ${ }^{12} \mathrm{CO} J=1-0$ line, all the ${ }^{13} \mathrm{CO}$ lines, and the $\mathrm{C}^{18} \mathrm{O}$ lines show U-shaped profiles typical of optically thin lines (Fig. 3). The emission of all the $\mathrm{CO}$ isotopologues is probably spatially resolved considering the typical beam size (Table 2) and the shape of the line profiles. The population diagrams of ${ }^{12} \mathrm{CO}$ and ${ }^{13} \mathrm{CO}$ hint small departures from a linear trend, owing to high optical depth and/or sub-thermal excitation (Fig. D.1). The results can be seen in Table 5 and Fig. 6 . We derived a fractional abundance $f\left({ }^{12} \mathrm{CO}\right) \gtrsim 1.1 \times 10^{-4}$ (with respect to $\mathrm{H}_{2}$ ) in agreement with previous estimates. For $\mathrm{C}^{18} \mathrm{O}$, we estimated $f\left(\mathrm{C}^{18} \mathrm{O}\right) \sim 4 \times 10^{-8}$, assuming the same excitation temperature $(20 \mathrm{~K})$ and emitting size as for ${ }^{13} \mathrm{CO}$.

We used MADEX to estimate the opacities of the CO lines (using the $T_{\text {kin }}$, the $v_{\text {exp }}$ derived from the linewidhts, the column density derived from the population diagram, and the $n\left(\mathrm{H}_{2}\right)$ density at the outer radius of the shell according to the equation given in Table 4). With these input parameters, MADEX predicts that the ${ }^{12} \mathrm{CO} J=2-1$ and the $J=3-2$ lines are moderately thick ( $\tau \lesssim 1.4$ ), while the $J=1-0$ line is optically thin $(\tau \sim 0.2)$. This is consistent with $\mathrm{CO}$ tracing the coolest, outermost layers of the $\mathrm{CSE}$ and ${ }^{13} \mathrm{CO}$ probing also regions deeper inside.

For $\mathrm{SiO}$ isotopologues we have adopted an emitting size equivalent to $7.9 \times 10^{15} \mathrm{~cm}\left(2^{\prime \prime}\right)$ at a distance of $265 \mathrm{pc}$ (Sect. 1.1). All the line profiles of the different $\mathrm{SiO}$ isotopologues are parabolic, consistent with optically thick emission. The rotational diagrams of all the $\mathrm{SiO}$ isotopologues display small departures from a linear trend, except perhaps for $\mathrm{Si}^{17} \mathrm{O}$ (Fig. D.2). We found similar $T_{\text {rot }}$ for all the isotopologues, that is, $T_{\text {rot }} \sim 20 \mathrm{~K}$, which are much lower than the $T_{\text {kin }}$ expected at a distance of $1^{\prime \prime}$ from the star (i.e. $T_{\text {kin }} \sim 105 \mathrm{~K}$ ). Hence, sub-thermal excitation may have an impact on the values derived from the population diagrams of $\mathrm{SiO}$ isotopologues.

MADEX (see Sect. 4.3) predicted $\tau>1$ for all of the $\mathrm{SiO}$, ${ }^{29} \mathrm{SiO}$ and ${ }^{30} \mathrm{SiO}$ lines (except for the $J=2-1$ lines of ${ }^{29} \mathrm{SiO}$ and ${ }^{30} \mathrm{SiO}$, for which MADEX predicted $\tau \lesssim 0.6$.) These values are consistent with the observed line profiles. For $\mathrm{Si}^{18} \mathrm{O}$ and $\mathrm{Si}^{17} \mathrm{O}$ lines, MADEX predicted $\tau<0.5$. Therefore, except for $\mathrm{Si}^{18} \mathrm{O}$ and $\mathrm{Si}^{17} \mathrm{O}$, the column densities and the abundances derived for the $\mathrm{SiO}$ isotopologues, given in Table 5, should be considered lower limits. The lower limit obtained, $f(\mathrm{SiO})>8.0 \times 10^{-6}$, is in good agreement with previous measures (Decin et al. 2010a, and references therein), and, in principle, is also consistent with the low $\mathrm{SiO}$ abundances predicted by the model by Gobrecht et al. (2016) that proposes the formation of $\mathrm{SiO}$ in abundance under thermodynamical equilibrium (TE) in the stellar photosphere, and a significant abundace decay (to $1.5 \times 10^{-5}$ ) already at $6 R_{*}$ mainly due to dust condensation.

\subsection{C-bearing molecules}

We detected emission of molecules, like CS, HCN and HNC, that are typically found in C-rich CSEs (e.g. Bujarrabal et al. 1994; Cernicharo et al. 2000; Zhang et al. 2009).

According to Marvel (2005), the HCN emission arises from a compact region with $\theta_{\mathrm{s}} \sim 4^{\prime \prime}$, which we adopted in this work. 
For CS, there are no observational constraints on the size of the emission, therefore, we adopted a size of $\theta_{\mathrm{s}}=2^{\prime \prime}$ which is the same size used for $\mathrm{SiO}$ and it is consistent with the extent of the CSE emission predicted by chemical models ( $\mathrm{Li}$ et al. 2016). Adopting these sizes we obtained $T_{\text {rot }}(\mathrm{HCN}) \sim 10 \mathrm{~K}$ and $T_{\text {rot }}(\mathrm{CS}) \sim 25 \mathrm{~K}$, and $N_{\text {tot }}(\mathrm{HCN}) \gtrsim 8 \times 10^{14} \mathrm{~cm}^{-2}$ and $N_{\text {tot }}(\mathrm{CS}) \sim$ $9 \times 10^{14} \mathrm{~cm}^{-2}$ (Figs. D.3 and D.4).

Using MADEX, we found optically thick lines $(\tau \gtrsim 1.5)$ for $\mathrm{HCN}$, and moderately thick lines $(\tau \lesssim 1.2)$ for $\mathrm{H}^{13} \mathrm{CN}$. Therefore, the column density and the abundance of $\mathrm{HCN}$ should be considered as lower limits. In the case of CS isotopologues, with the physical conditions expected at $r \sim 1^{\prime \prime}$, MADEX predicted optically thin lines $(\tau \lesssim 1.0)$. Finally, given that $T_{\mathrm{kin}}(r \lesssim$ $\left.1^{\prime \prime}\right) \gtrsim 100 \mathrm{~K}$, the lines of HCN and CS isotopologues are most likely sub-thermally excited.

The rotational temperatures and abundances derived for $\mathrm{HCN}$ and CS isotopologues (see Table 5) are consistent with previous measurements in IK Tau (Lindqvist et al. 1988; Kim et al. 2010). The HCN abundance is in the high end of the abundance range deduced by Schöier et al. (2013) in a sample of M-type AGB stars. The TE models for O-rich CSEs do not account for the HCN and CS abundances observed in O-rich CSEs, predicting values of $f(\mathrm{HCN}) \sim 10^{-11}$ and $f(\mathrm{CS}) \sim$ $10^{-10}$ (Duari et al. 1999; Gobrecht et al. 2016). The inclusion of shocks can contribute to enhance the formation of $\mathrm{HCN}$ in O-rich stars (Duari et al. 1999; Cherchneff 2006; Gobrecht et al. 2016), but it also brings up a theoretical homogeinity on the expected HCN abundances among different chemical types of stars that it is not observed, as noted by Schöier et al. (2013). Other authors invoked the photochemistry to try to explain the abundances of C-bearing molecules observed in O-rich CSEs (Nercessian et al. 1989; Ziurys et al. 2009). In particular, the chemical model of Willacy \& Millar (1997) predicts peak abundances of $f(\mathrm{HCN}) \sim 1 \times 10^{-7}$ and $f(\mathrm{CS}) \sim 3 \times 10^{-7}$, although the carbon source proposed by these authors is $\mathrm{CH}_{4}$ which has been later on refuted (Marvel 2005). Additionally, it has been also proposed that CSEs could be clumpy, hence, photochemistry could be important also in the inner layers of the envelopes (Agúndez et al. 2010). Our results do not conclude clearly which is the most likely scenario, although, our derived abundances are more similar to those predicted by the models of Gobrecht et al. (2016).

Finally, we detected two HNC lines, in particular, the $J=$ $1-0$, and the $J=3-2$ line, which is blended with the image of the SiO $J=6-5$ line. We estimated $f \sim 8 \times 10^{-9}$, assuming an excitation temperature of $\sim 30 \mathrm{~K}$ and a size of $2^{\prime \prime}$ for the emitting region, which are average values for these parameters.

\subsection{Refractory species}

We confirmed the presence of two important refractory molecules which are mainly found in C-rich envelopes: SiS and $\mathrm{NaCl}$. The emission of these molecules has not been mapped in previous studies and, therefore, the size of the emitting region is unknown. The $\mathrm{NaCl}$ line profiles, with $v_{\exp } \sim 14 \mathrm{~km} \mathrm{~s}^{-1}$, are narrower than those of SiS, consistent with $v_{\exp } \sim 18.5 \mathrm{~km} \mathrm{~s}^{-1}$, which suggests a more inner distribution of $\mathrm{NaCl}$ around the star (see Fig. 7). This molecule may condense onto the dust grains beyond the dust condensation radius, as proposed by Milam et al. (2007). The profiles of the lines of $\mathrm{NaCl}$ and $\mathrm{SiS}$ isotopologues indicate spatially unresolved emission, which is compatible with the Gaussian-like profiles observed in the case of $\mathrm{NaCl}$, and the triangular or parabolic profiles observed for the SiS isotopologues. Moreover, the Gaussian-like profiles observed for $\mathrm{NaCl}$ may support that the emission of this molecule arises from the innermost regions of the CSE, where the gas has not been fully accelerated.

We adopted a size of $\theta_{\mathrm{s}}=2^{\prime \prime}$ for $\mathrm{SiS}$ isotopologues, as a first guess considering the emission size of $\operatorname{SiO}\left(\theta_{\mathrm{s}}=2^{\prime \prime}\right)$. This size is also consistent with the size predicted by recent chemical model of IK Tau ( $\mathrm{Li}$ et al. 2016). For $\mathrm{NaCl}$ we adopted a size of $\theta_{\mathrm{s}}(\mathrm{NaCl})=0 .{ }^{\prime \prime} 3$ (Milam et al. 2007). We derived similar rotational temperatures for $\mathrm{SiS}$ and $\mathrm{NaCl}$ (i.e. $T_{\text {rot }} \sim 65 \mathrm{~K}$ ) even higher for ${ }^{29} \mathrm{SiS},{ }^{30} \mathrm{SiS}$, and $\mathrm{Si}^{34} \mathrm{~S}$ (see Figs. D.5 and D.6). According to the size adopted for SiS (i.e. $r \lesssim 4 \times 10^{15} \mathrm{~cm}$ ), we estimated $T_{\text {kin }} \gtrsim 105 \mathrm{~K}$, and $n\left(\mathrm{H}_{2}\right) \gtrsim 3.5 \times 10^{5} \mathrm{~cm}^{-3}$. For the size adopted for $\mathrm{NaCl}$ (i.e. $r \lesssim 6 \times 10^{14} \mathrm{~cm}$ ), we estimated $T_{\text {kin }} \gtrsim 330 \mathrm{~K}$. Hence, $\mathrm{SiS}$ and $\mathrm{NaCl}$ are most likely sub-thermally excited. We estimated critical densities for the SiS lines of $n_{\text {crit }} \sim\left[10^{4}-10^{6}\right] \mathrm{cm}^{-3}$ for a temperature of $\sim 105 \mathrm{~K}$, therefore, $n \lesssim n_{\text {crit }}$ for several lines of SiS confirming sub-thermal excitation. For $\mathrm{NaCl}$ the critical densities expected are even higher, $n_{\text {crit }} \gtrsim 5 \times 10^{7} \mathrm{~cm}^{-3}$, due to the high dipole moment of $\mathrm{NaCl}$. MADEX predicted optically thin lines for both $\operatorname{SiS}(\tau<0.6)$ and $\mathrm{NaCl}(\tau<0.3)$.

We derived $f(\mathrm{SiS}) \sim 5 \times 10^{-6}$. This value is in good agreement with the estimations by Kim et al. (2010). The chemical model by Gobrecht et al. (2016) predicts a SiS abundance of $4 \times 10^{-8}$ under TE and up to $\sim 3 \times 10^{-7}$ including dust condensation and shocks due to the pulsation of the star, which is at least one order of magnitude lower than our results. Willacy \& Millar (1997) used SiS in their chemical models as a parent molecule with an abundance consistent with our observations (see Table 5).

Concerning $\mathrm{NaCl}$, Milam et al. (2007) derived a rotational temperature and a column density consistent with our results. However, Milam et al. (2007) derived a fractional abundance $\sim 80$ times lower than ours, through the population diagram of two low $\mathrm{S} / \mathrm{N} \mathrm{NaCl}$ emission lines, and also a radiative transfer calculation using the code by Bieging \& Tafalla (1993), with a set of $\mathrm{SiO}$-corrected collisional coefficients. We detected $13 \mathrm{NaCl}$ lines which cover a wide range in $E_{\mathrm{u}}$ and have better $\mathrm{S} / \mathrm{N}$, from which we derived an average fractional abundance of $f(\mathrm{NaCl})=3 \times 10^{-7}$ (see Table 5 and Fig. D.6). We cannot rule out uncertainties in our estimation due to the emitting size, and the ${ }^{13} \mathrm{CO}$ column density adopted, which could not be representative in the region of $\mathrm{NaCl}$ emission. Moreover, $\mathrm{NaCl}$ line profiles are not incompatible with $\theta_{\mathrm{s}}(\mathrm{NaCl}) \gtrsim 0$.' 3 , in particular, if $\theta_{\mathrm{s}}(\mathrm{NaCl})=1^{\prime \prime}$ we would derive $f(\mathrm{NaCl})=3 \times 10^{-8}$. TE calculations predict abundances of $10^{-11}$ up to $10^{-7}$ (Tsuji 1973; Milam et al. 2007), while Gobrecht et al. (2016) models ( $\mathrm{TE}$ and shocks) predicts $\mathrm{NaCl}$ abundances between $4 \times 10^{-12}$ up to $1 \times 10^{-8}$. It would be necessary to obtain maps of the $\mathrm{NaCl}$ spatial distribution in order to clarify these discrepancies.

\subsection{S-bearing molecules}

Here we discuss the detected emission of $\mathrm{H}_{2} \mathrm{~S}, \mathrm{SO}$, and $\mathrm{SO}_{2}$. The first detection of these molecules toward IK Tau and their chemistry in O-rich CSEs was presented in Omont et al. (1993) and references therein. Omont et al. (1993) only detected one line of $\mathrm{H}_{2} \mathrm{~S}$, and they were not able to estimate its abundance toward IK Tau. The emission of $\mathrm{SO}$ and $\mathrm{SO}_{2}$ molecules in O-rich CSEs, including IK Tau, has been recently reviewed and modelled by Danilovich et al. (2016).

We detected three ortho and one para lines of $\mathrm{H}_{2} \mathrm{~S}$ as well as one line of $\mathrm{o}-\mathrm{H}_{2}{ }^{34} \mathrm{~S}$, which point out $v_{\exp } \sim v_{\infty}$. The profiles of the 

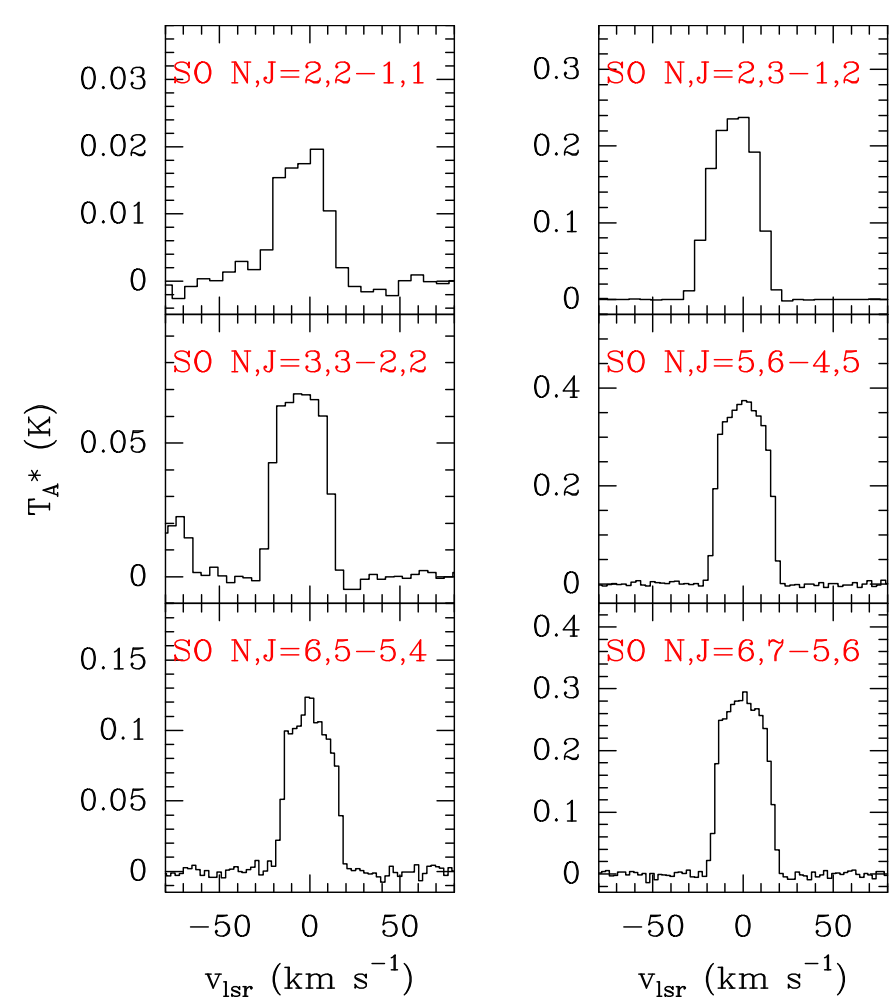

Fig. 10. Line profiles of some of the SO lines identified. The quantum numbers of each transition are plotted in red in each box.The spectral resolution is $2 \mathrm{MHz}$ for all the spectra shown.

lines indicate spatially unresolved emission. Since there are not maps of the $\mathrm{H}_{2} \mathrm{~S}$ emission, we adopted a size of $\theta_{\mathrm{s}}\left(\mathrm{H}_{2} \mathrm{~S}\right)=2^{\prime \prime}$ which is consistent with the size predicted by chemical models ( $\mathrm{Li}$ et al. 2016). Given that we only detected two lines of o- $\mathrm{H}_{2} \mathrm{~S}$ with $\mathrm{S} / \mathrm{N}>5$, we calculated the population diagram (Fig. D.7) of ortho and para species together adopting an orthoto-para ratio of 3:1 which is the value that could be expected from the formation processes of $\mathrm{H}_{2} \mathrm{~S}$. We derived $T_{\text {rot }} \sim 40 \mathrm{~K}$ and $f\left(\mathrm{H}_{2} \mathrm{~S}\right) \sim 1 \times 10^{-6}$. This value is consistent with the chemical models presented by Gobrecht et al. (2016) at a few stellar radii. We found that the lines of $\mathrm{H}_{2} \mathrm{~S}$ are likely to be sub-thermally excited at the distances adopted for the $\mathrm{H}_{2} \mathrm{~S}$ emission $\left(r \lesssim 1^{\prime \prime}\right)$. However, we did not made further non-LTE calculations due to the lack of a set of collisional coefficients for $\mathrm{H}_{2} \mathrm{~S}$. Under LTE conditions, MADEX predicts optically thin lines $(\tau<0.6)$.

The profiles of the SO lines observed are varied (see Fig. 10). Most of them are flat-topped (optically thin and spatially unresolved emission), some of them display parabolic profiles (optically thick or moderately thick emission) and a few SO lines display profiles which seem to be composed of two components, at least: one dominant flat-topped or parabolic component with linewidths consistent with $v_{\infty}$, and an additional narrow feature which may indicate SO gas inside the gas acceleration region. These two components are more clearly seen in several $\mathrm{SO}_{2}$ lines (Fig. 11) which display a broad component plus a bulge-like centred narrow component, which we interpreted as $\mathrm{SO}_{2}$ emission arising from $r<8 R_{*}$ (see Sect. 6.5.1). Nevertheless, we have not detected high- $E_{\mathrm{u}}$ SO narrow lines or two different trends in the SO population diagram (see below) which could prove a very inner component of warm SO gas (contrary to $\mathrm{SO}_{2}$ as discussed in Sect. 6.5.1). Thus we have no firm evidence that could prove the presence of warm SO gas in the innermost regions of the CSE with a noticeable abundance. Finally, the ${ }^{34}$ SO flat-topped (optically thin emission) profiles yield also $v_{\exp } \sim v_{\infty}$.
The brightness distribution of SO has not been mapped before, thus, we adopted a size of $\theta_{\mathrm{s}}(\mathrm{SO})=2^{\prime \prime}$ as well as for ${ }^{34} \mathrm{SO}$, according to the models by Li et al. (2016). The population diagrams (Fig. D.8) display departures from a linear trend for SO. We obtained $T_{\text {rot }}(\mathrm{SO}) \sim T_{\text {rot }}\left({ }^{34} \mathrm{SO}\right) \sim 15 \mathrm{~K}, N_{\text {tot }}(\mathrm{SO}) \gtrsim$ $9 \times 10^{15} \mathrm{~cm}^{-2}$ and $\left.N_{\text {tot }}{ }^{34} \mathrm{SO}\right) \sim 9 \times 10^{14} \mathrm{~cm}^{-2}$. We verified with MADEX (see Sect. 4.3) that SO lines would be moderatelly thick $(\tau \lesssim 1.5)$ with a $T_{\text {kin }}=105 \mathrm{~K}$ and $n \sim 4 \times 10^{5} \mathrm{~cm}^{-3}$ at $r=1^{\prime \prime}$. MADEX predicted optically thin lines for ${ }^{34} \mathrm{SO}(\tau \lesssim 0.2)$. Furthermore, we estimated $n_{\text {crit }}(\mathrm{SO}) \sim\left[10^{5}-10^{7}\right] \mathrm{cm}^{-3}$ and similar values for ${ }^{34} \mathrm{SO}$, which suggests sub-thermal excitation of several transitions.

We derived abundances of $f(\mathrm{SO}) \gtrsim 8 \times 10^{-6}$ and $f\left({ }^{34} \mathrm{SO}\right) \sim$ $8 \times 10^{-7}$. The abundance measured of SO is at least a factor three higher compared to previous observational works toward IK Tau (Omont et al. 1993; Bujarrabal et al. 1994; Kim et al. 2010). On the other hand, TE models predict abundances of $f(\mathrm{SO}) \sim[2-4] \times 10^{-8}$ (Duari et al. 1999; Gobrecht et al. 2016). Willacy \& Millar (1997) derived peak abundances up to $f(\mathrm{SO}) \sim$ $9 \times 10^{-7}$ with a chemical model for an O-rich CSE that used only $\mathrm{H}_{2} \mathrm{~S}$ and $\mathrm{SiS}$ as parent $\mathrm{S}$-bearing molecules. Compared to these models, our derived abundance is at least nine times higher than the highest value obtained from the models. Our analysis seems to overestimate the SO abundance compared to previous measurements and chemical models. These dicrepancies may be explained given the uncertainty on the size of the SO emitting region adopted, and the $f\left({ }^{13} \mathrm{CO}\right)$ adopted (see Eq. (9)). In particular, a size of $\theta_{\mathrm{s}}(\mathrm{SO}) \sim 5^{\prime \prime}$ would fix this discrepancy.

\subsection{1. $\mathrm{SO}_{2}$ and ${ }^{34} \mathrm{SO}_{2}$}

We detected $\sim 90$ lines of $\mathrm{SO}_{2}$ displaying complex profiles which can be grouped according to their $v_{\text {exp }}$ : (i) $\sim 60$ lines with $v_{\exp } \sim 18 \mathrm{~km} \mathrm{~s}^{-1}$ consistent with the $v_{\infty}$ of the CSE; and (ii) $\sim 30$ lines with $v_{\text {exp }}<v_{\infty}$, with velocities as low as $\sim 5 \mathrm{~km} \mathrm{~s}^{-1}$ (see Fig. 7). The lines of $\mathrm{SO}_{2}$ display parabolic profiles, flattopped profiles, and complex profiles. Several lines display a self-absorption in the blue side of the line (see Fig. 11). This selfabsorption may be explained considering that part of the $\mathrm{SO}_{2}$ emission arising from the inner and warm shells of the CSE, is absorbed by the external and cold shells of the CSE, which are located (within the line of sight) between us and the warm gas. The narrow lines seem to be spatially unresolved, although, for the lines that have $v_{\exp } \sim v_{\infty}$ it is not clear whether they are spatially resolved or not. Most of the ${ }^{34} \mathrm{SO}_{2}$ lines show flat-topped profiles with indicative of spatially unresolved emission.

The rotational diagram of $\mathrm{SO}_{2}$ was done adopting an emission size of $\theta_{\mathrm{s}}\left(\mathrm{SO}_{2}\right)=2^{\prime \prime}$, which should be considered as an educated guess derived from the chemical models by Li et al. (2016). From the population diagram of $\mathrm{SO}_{2}$ (Fig. D.9) we observed also two components, a cold component with $T_{\text {rot }} \sim 40 \mathrm{~K}$ traced out by $\sim 60$ lines with $E_{\mathrm{u}} \lesssim 160 \mathrm{~K}$ and a warm component with $T_{\text {rot }} \sim 290 \mathrm{~K}$ traced out by $\sim 30$ lines with $E_{\mathrm{u}}>160 \mathrm{~K}$. The cold component displays a slight change in the trend for the lines with $E_{\mathrm{u}} \lesssim 50 \mathrm{~K}$ which may be explained as a result of moderate optically thick emission for those lines and/or sub-thermal excitation. Using Eq. (9) we derived $f\left(\mathrm{SO}_{2}\right.$, cold $) \gtrsim 9.6 \times 10^{-6}$ and $f\left(\mathrm{SO}_{2}\right.$, warm $) \gtrsim 2.7 \times 10^{-6}$. For ${ }^{34} \mathrm{SO}_{2}$ we derived a $T_{\text {rot }} \sim 35 \mathrm{~K}$ and $f\left({ }^{34} \mathrm{SO}_{2}\right) \sim 9.6 \times 10^{-7}$.

As we said in Sect.4.3, the $\mathrm{SO}_{2}$ lines detected span over a wide range of energies, thus, the parameters derived from the population diagram may be unreliable given that the 

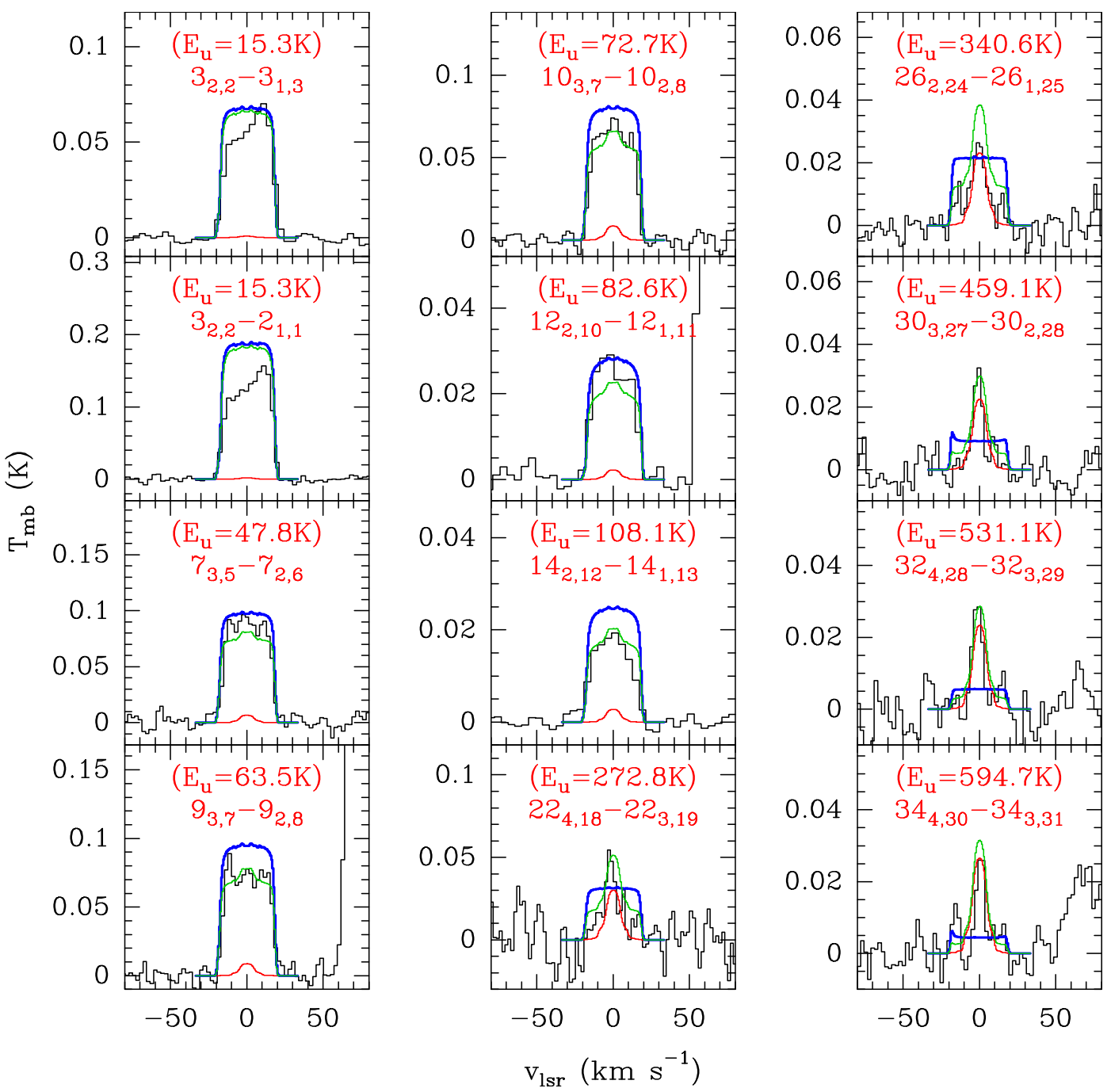

Fig. 11. Results of the radiative transfer models of $\mathrm{SO}_{2}$ explained in Sects. 4.3 and 6.5.1. The coloured curves correspond to the models adopting the abundance profiles shown in Fig. 5. The spectral resolution is $2 \mathrm{MHz}$. The temperature scale is in main beam temperature.

homogenous temperature assumption may turn out to be a very crude approximation. We carried out several LVG models adopting different radial abundance profiles (see Sect. 4.3 and Fig. 5). As it is discussed below, the different abundance profiles were adopted mainly to illustrate the need of the presence of warm $\mathrm{SO}_{2}$ at $r<8 R_{*}$ in order to reproduce the profiles of the high- $E_{\mathrm{u}}$ lines. The results of the radiative transfer models are shown in Fig. 11 for several lines of $\mathrm{SO}_{2}$.

The first model (red line in Figs. 5 and 11) reproduces to a good accuracy the profiles of the narrow high- $E_{\mathrm{u}}$ (i.e. $E_{\mathrm{u}}>160 \mathrm{~K}$ ) lines observed. However, it is unable to reproduce the line profiles of the low- $E_{\mathrm{u}}$ (i.e. $E_{\mathrm{u}}<160 \mathrm{~K}$ ) lines, underestimating their emission completely. We created a second model (blue line in Figs. 5 and 11) which reproduces, within a factor two in intensity, most of the low- $E_{\mathrm{u}} \mathrm{SO}_{2}$ lines observed. However, this model is unable to explain the narrow profiles of the high- $E_{\mathrm{u}} \mathrm{SO}_{2}$ lines, predicting wide flat-topped lines or even no emission for these high energy lines. We may note, that the fractional abundance adopted for this second model is approximately a factor five lower than the abundance derived from the population diagram of the $\mathrm{SO}_{2}$ cold component. If the abundance is increased up to a value consistent with the population diagram results for the cold component (i.e. $\left.f\left(\mathrm{SO}_{2}\right)=9.6 \times 10^{-6}\right)$, the model highly overestimates the line profiles observed. This discrepancy may arise from the lack of precise information about the spatial distribution of $\mathrm{SO}_{2}$ toward the CSE.

Finally, we tested the possibility of $\mathrm{SO}_{2}$ being distributed as a sum of two components, in particular, the sum of a compact inner component plus an extended component with a shell-like enhancement in the outermost part of the CSE, in order to reproduce the whole set of $\mathrm{SO}_{2}$ lines observed with a single radial abundance profile. This last model (green line in Figs. 5 and 11) is able to approximately reproduce both the low- and high- $E_{\mathrm{u}}$ $\mathrm{SO}_{2}$ lines observed, although, it does not reproduces perfectly all the line profiles. The discrepancies found between this best model and the observations are within a factor two or three in intensity for most of the lines observed. This disagreement emerges probably from the lack of precise information about the spatial distribution of $\mathrm{SO}_{2}$. The radial abundance profile adopted for the model is consistent, within a factor two or three in intensity, with the results obtained from the population diagram for the $\mathrm{SO}_{2}$ warm and cold components. Additionally, the best model predicts both optically thin and optically thick lines which is consistent with the variety of profiles observed.

Hence, we can conclude that $\mathrm{SO}_{2}$ is distributed along the $\mathrm{CSE}$ with an average fractional abundance of $f\left(\mathrm{SO}_{2}\right) \sim 10^{-6}$. 
Our models evidenced the presence of an inner (1-8 $R_{*}$ ) warm ( $2290 \mathrm{~K}$ ) component of $\mathrm{SO}_{2}$ with fractional abundances $\sim 10^{-6}$, which produces most of the emission from $\mathrm{SO}_{2}$ lines with $E_{\mathrm{u}} \gtrsim 160 \mathrm{~K}$. This is also consistent with the narrow profiles of the high- $E_{\mathrm{u}} \mathrm{SO}_{2}$ lines. Nevertheless, our model is not able to reproduce the complexity observed in the profiles, which probably indicates the simplicity of the approximated physical model. In particular, the outer radius of the $\mathrm{SO}_{2}$ cold component is critical to control the expected intensities of the low- $E_{\mathrm{u}}$ lines, which we adopted from the chemical model by Li et al. (2016). Therefore, it would be necessary to map the brightness distribution of this molecule in order to constrain the outer radius of $\mathrm{SO}_{2}$ emission. High angular resolution observations are also required to map the innermost regions of the CSE, since the distribution of $\mathrm{SO}_{2}$ in the region between $1-20 R_{*}$ would improve the results obtained not only for the high- $E_{\mathrm{u}}$ lines but also for those lines that display a narrow core component (e.g. $\mathrm{SO}_{2} 7_{3,5}-7_{2,6}$ in Fig. 11).

Previous works toward IK Tau pointed out $\mathrm{SO}_{2}$ abundances in the intermediate and outer envelope consistent with our results (Omont et al. 1993; Kim et al. 2010; Decin et al. 2010a). Decin et al. (2010a) hinted the presence of $\mathrm{SO}_{2}$ in the inner wind, since they were not able to reproduce with their radiative transfer model simultaneously the emission of a few lines with $E_{\mathrm{u}} \sim 140 \mathrm{~K}$ detected with APEX and the low- $E_{\mathrm{u}}$ transitions observed with the IRAM-30 m telescope. Given the limited number of high- $E_{\mathrm{u}}$ lines detected by Decin et al. (2010a), these authors were unable to reach conclusive results on the presence of $\mathrm{SO}_{2}$ in the inner wind of IK Tau and its abundance. Recent research was conducted to investigate one $\mathrm{SO}_{2}$ line with $E_{\mathrm{u}} \sim 600 \mathrm{~K}$ detected with Herschel/HIFI as well as other $\mathrm{SO}_{2}$ lines reported in the literature toward IK Tau with a radiative transfer model by Danilovich et al. (2016). Their best-fit model has a peak abundance of $f\left(\mathrm{SO}_{2}\right)=2 \times 10^{-6}$ and an $e$-folding radius $R_{\mathrm{e}}=10^{16} \mathrm{~cm}$, although, it is unable to reproduce all the $\mathrm{SO}_{2}$ observed line profiles as indicated by these authors.

TE models predict the formation of $\mathrm{SO}_{2}$ in the photosphere of the star with abundances of $f\left(\mathrm{SO}_{2}\right) \sim 10^{-11}$ (Tsuji 1973; Gobrecht et al. 2016), which is approximately five orders of magnitude lower than our results. Willacy \& Millar (1997) predicted a peak abundance of $f\left(\mathrm{SO}_{2}\right) \sim 2 \times 10^{-7}$ in the intermediate and outer parts of the CSE, at $r \sim 10^{16} \mathrm{~cm}$ (i.e. $\sim 500 R_{*}$ ), and $f\left(r \lesssim 3 \times 10^{15} \mathrm{~cm}\right) \lesssim 10^{-10}$, where they used $\mathrm{H}_{2} \mathrm{~S}$ and $\mathrm{SiS}$ as the S-bearing parent molecules of the model. This chemical model is also inconsistent with our results given that they do not predict the formation of $\mathrm{SO}_{2}$ in the inner parts of the CSE and the abundance in the intermediate and outer parts is aprroximately two orders of magnitude lower than our measures. In the recent chemical model presented by Li et al. (2016), the authors explore the effect of including $\mathrm{SO}_{2}$ as a parent molecule. In the absence of a reliable observational estimate of the $\mathrm{SO}_{2}$ abundance in the inner envelope, these authors adopt $f\left(\mathrm{SO}_{2}\right)=2 \times 10^{-6}$, which is the value estimated from low- $E_{\mathrm{u}} \mathrm{SO}_{2}$ transitions arising in the outer envelope regions (Decin et al. 2010a). In the innermost parts of the envelope $\left(r<8 R_{*}\right)$ the $\mathrm{SO}_{2}$ abundance can be enhanced up to $f\left(\mathrm{SO}_{2}\right)=4 \times 10^{-9}$ including the effect of shocks and dust grains (Gobrecht et al. 2016). These authors suggest that the production of $\mathrm{SO}$ triggers the formation of $\mathrm{SO}_{2}$ at $\sim 4 R_{*}$ in the gas phase through the reaction with $\mathrm{OH}$. Although, the $\mathrm{SO}_{2}$ abundance obtained with the inclussion of shocks is approximately two or three orders of magnitude lower than our estimates. Photochemistry may also enhance the formation of $\mathrm{SO}_{2}$ in the inner layers of the CSE, which would require an additional source of UV radiation to dissociate $\mathrm{H}_{2} \mathrm{O}$ providing $\mathrm{OH}$ to react with the $\mathrm{SO}$ formed leading to the enhancement of $\mathrm{SO}_{2}$. This could be plausible if the envelope of IK Tau is clumpy, as proposed for other objects (Agúndez et al. 2010).

\section{6. $N$-bearing molecules}

Besides $\mathrm{HCN}, \mathrm{HNC}$ and $\mathrm{PN}$, which are discussed in other sections, we detected $\mathrm{CN}$, NS, and NO. The $\mathrm{CN}$ lines with unblended hyperfine components have widths consistent with $v_{\text {exp }}=v_{\infty}$ within errors. The spatial distribution of CN in IK Tau is unknown. $\mathrm{CN}$ has been observed in the outer shells of the C-rich CSE IRC+10216 (Lucas et al. 1995), and chemical models predict that it is formed as a result of the photodissociation of HCN and HNC in these outer shells (e.g. Nejad \& Millar 1988). Given that the $\mathrm{HCN}$ size is $\theta_{\mathrm{s}}(\mathrm{HCN})=33^{\prime \prime} 85, \mathrm{CN}$ would be expected to be in a shell external to the HCN. According to the chemical model by Li et al. (2016), the CN peak abundance occurs at $r \sim 1.5 \times 10^{16} \mathrm{~cm}$. We converted the area between the $\mathrm{HCN}$ outer shell and the $\mathrm{CN}$ abundance peak to an equivalent emitting size, obtaining $\theta_{\mathrm{s}}(\mathrm{CN}) \sim 6^{\prime \prime}$. With this size, we calculated the population diagram of $\mathrm{CN}$ (Fig. D.10). We estimated $T_{\text {rot }}=9 \pm 2$ and $N_{\text {tot }} \sim 1 \times 10^{14} \mathrm{~cm}^{-2}$. At $r \sim 1.5 \times 10^{16} \mathrm{~cm}, T_{\text {kin }}$ is $\sim 50 \mathrm{~K}$ and $n\left(\mathrm{H}_{2}\right) \sim 2 \times 10^{4} \mathrm{~cm}^{-3}$, thus, CN lines are probably sub-thermally excited $\left(n_{\text {crit }} \gtrsim 10^{6} \mathrm{~cm}^{-3}\right.$ ). With these physical conditions MADEX predicted optically thin lines. We estimated $f(\mathrm{CN}) \sim 1 \times 10^{-7}$ which is consistent with previous estimations (Kim et al. 2010). Chemical models predict abundances up to $3 \times 10^{-7}$ (Willacy \& Millar 1997; Li et al. 2016), which are also in good agreement with our observations.

As far as we know, our discovery of NS emission toward IK Tau is the first detection of this molecule in this source. Given that we did not resolve its hyperfine structure we cannot extract information on the line profiles observed. There are not observational constraints on the emission size of this molecule. According to the chemical model by Li et al. (2016), which predicts that NS would be formed through the neutral-neutral reaction of $\mathrm{NH}$ and $\mathrm{S}$ in an external shell of the envelope, similar to the $\mathrm{CN}$ shell, we adopted a $\theta_{\mathrm{s}}(\mathrm{NS}) \sim 6^{\prime \prime}$. With this size, from its population diagram (Fig. D.11), we derive a $T_{\text {rot }}=18 \pm 2 \mathrm{~K}$ and a column density of $(2.6 \pm 0.5) \times 10^{13} \mathrm{~cm}$. As for $\mathrm{CN}$, the lines of NS may be sub-thermally excited. Since, we have not a set of collisional coefficients for NS we could neither estimate the opacities of the lines nor their critical densities. A rough estimation under LTE conditions with MADEX yields optically thin lines $(\tau<0.1)$. We derived an abundance of $f(\mathrm{NS}) \sim 2 \times 10^{-8}$. The chemistry of NS in O-rich CSEs was discussed in Willacy \& Millar (1997), although, these authors did not give a value for the predicted NS abundance. Recently, chemical models by Li et al. (2016) predicted $f(\mathrm{NS}) \sim 8 \times 10^{-9}$, which is (within uncertainties) consistent with our results.

For NO, we only detected two lines with low $\mathrm{S} / \mathrm{N}$. One of them is a blend of several hyperfine components. For the NO hyperfine component spectrally resolved we derived $v_{\exp }=v_{\infty}$. We estimated a rough value of the NO abundance adopting $\theta_{\mathrm{s}} \sim 6^{\prime \prime}$ (like for $\mathrm{CN}$ and NS), and an excitation temperature of $30 \mathrm{~K}$, which is representative of the $T_{\text {kin }}$ in the outer shells of the CSE $\left(r \sim[2-5] \times 10^{16} \mathrm{~cm}\right)$. With these considerations, we obtained $f(\mathrm{NO}) \sim 2 \times 10^{-6}$, in agreement with the predictions of the chemical model by Li et al. (2016).

\subsection{P-bearing molecules}

PN and PO were detected for the first time toward IK Tau by De Beck et al. (2013), and we detected one additional line of 
PN, and seven additional lines of PO. Concerning PO, which has hyperfine structure, we observed several spectrally resolved (as well as unresolved) lines with linewidths consistent with $v_{\exp } \sim 9 \mathrm{~km} \mathrm{~s}^{-1}$. This suggests that PO emission lines arise from $r<8 R_{*}$. De Beck et al. (2013) mapped the brightness distribution of both PN and PO toward IK Tau, and found $\theta_{\mathrm{s}} \lesssim 0$.' 7 . With this size we calculated the rotational diagram of both molecules (Fig. D.12), and we derived low rotational temperatures $\left(T_{\text {rot }} \sim 20 \mathrm{~K}\right), N_{\text {tot }}(\mathrm{PN}) \sim 8 \times 10^{14} \mathrm{~cm}^{-2}$ and $N_{\text {tot }}(\mathrm{PO}) \sim$ $2 \times 10^{15} \mathrm{~cm}^{-2}$. The low $T_{\text {rot }}$ deduced for PN and PO is probably indicative of sub-thermal excitation since at the inner wind layers $\left(<0.7^{\prime \prime}\right)$ where the emission is produced, the gas kinetic temperature is expected to be well above $200 \mathrm{~K}$. We used our radiative transfer code (see Sect.4.3) to confirm our results. MADEX predicted optically thin $(\tau \lesssim 0.4)$ and sub-thermally excited lines for PN. In the case of PO, MADEX predicted optically thin $(\tau<0.1)$ PO lines, under LTE approximation given that there is not a set of PO collisional coefficients available. We derived $f(\mathrm{PN}) \sim 7 \times 10^{-7}$ and $f(\mathrm{PO}) \sim 2 \times 10^{-6}$, which are consistent with previous estimates considering uncertainties (De Beck et al. 2013).

Concerning chemical models, these P-bearing molecules are adopted as parent molecules and their abundances are assumed from observations of the inner region of the CSE (De Beck et al. 2013; Li et al. 2016). TE calculations predict abundances for PN compatible with our results, and one order of magnitude lower than our measurements for PO (Tsuji 1973; Agúndez et al. 2007; Milam et al. 2008).

\section{8. $\mathrm{HCO}^{+}$}

We detected the $J=1-0$ line of the $\mathrm{HCO}^{+}$ion. The $J=3-2$ line was not detected probably due to an unsufficient sensitivity. The $J=2-1$ and the $J=4-3$ lines lie in wavelength ranges that were not observed. The flat-topped profile of the $\mathrm{HCO}^{+} J=1-0$ line indicates $v_{\exp } \sim v_{\infty}$, optically thin, and spatially unresolved emission. We estimated a very rough value of the $f\left(\mathrm{HCO}^{+}\right)$adopting an emission region and an excitation temperature equal to those adopted for NO (see Sect. 6.6) since both molecules are expected to be formed in the outer shells of the CSE. With these values, we obtained $f\left(\mathrm{HCO}^{+}\right) \sim 10^{-8}$.

According to chemical models, this molecule is formed efficiently in the outer layers of O-rich CSEs as a result of reactions that involve $\mathrm{CO}, \mathrm{H}_{2} \mathrm{O}$, and their photodissociation products, with abundances consistent with our results (Willacy \& Millar 1997; Sánchez Contreras et al. 2015, and references therein).

\subsection{The organic precursor missing link to carbon chemistry: $\mathrm{H}_{2} \mathrm{CO}$}

This is the first detection of the organic precursor molecule $\mathrm{H}_{2} \mathrm{CO}$ toward IK Tau. We detected ortho and para lines with flattopped profiles, indicating optically thin and spatially unresolved emission, even for the lines at high frequencies with $\theta_{\mathrm{b}} \sim 8^{\prime \prime}$ (see Eq. (4)). We adopted a size of $\theta_{\mathrm{s}}\left(\mathrm{H}_{2} \mathrm{CO}\right)=2^{\prime \prime}$, which is an educated guess, taking into account that most of the molecules detected are expected to emit in that region of the CSE.

In the population diagram of $\mathrm{H}_{2} \mathrm{CO}$ (see Fig. D.13), the $K_{\mathrm{a}}=0, K_{\mathrm{a}}=1$, and $K_{\mathrm{a}}=2$ ladders were fitted separately. For $K_{\mathrm{a}}=2$ only two data were collected. The average rotational temperature indicated by the fits of the different $K_{\mathrm{a}}$ ladders is $\sim 10 \mathrm{~K}$. For the $K_{\mathrm{a}}=3$ ladder we only detected two lines with the same upper energy level, therefore, we adopted an average excitation temperature of $\sim 10 \mathrm{~K}$ to derive a rough value of the $K_{\mathrm{a}}=3$ column density. Formaldehyde is an asymmetric rotor with its dipolar moment oriented along the $a$ axis. Therefore, transitions between different $K_{\mathrm{a}}$ levels are weakly connected through radiative processes. This explains why each of the $K_{\mathrm{a}}$ ladders appears as separated lines in the rotational diagram. The column densities derived result in an ortho-to-para ratio of $\sim 3: 1$, which has been computed dividing the sum of the $K_{\mathrm{a}}=1$ and 3 (ortho transitions) by the sum of the $K_{\mathrm{a}}=0$ and 2 (para transitions) column densities (see Fig. D.13). The total column density is $N_{\text {tot }}\left(\mathrm{H}_{2} \mathrm{CO}\right) \sim 3 \times 10^{14} \mathrm{~cm}^{-2}$. Adding both $\mathrm{o}-\mathrm{H}_{2} \mathrm{CO}$ and $\mathrm{p}-\mathrm{H}_{2} \mathrm{CO}$, we obtained a fractional abundance of $f\left(\mathrm{H}_{2} \mathrm{CO}\right) \sim 2 \times 10^{-7}$. In this case, MADEX predicted optically thin lines $(\tau<0.3)$ and sub-thermal excitation which is consistent with $T_{\text {kin }}\left(r=1^{\prime \prime}\right)>T_{\text {rot }}$.

The origin of formaldehyde, as well as other C-bearing species, in O-rich envelopes has puzzled the scientific community since $\mathrm{H}_{2} \mathrm{CO}$ was first detected in the O-rich CSE OH231.8+4.2, with a fractional abundance of $4 \times 10^{-8}$ (Lindqvist et al. 1992; Charnley et al. 1995). Millar \& Olofsson (1993) proposed a formation route which requires methane to produce formaldehyde in the external envelope with abundances up to $10^{-7}$ depending on the mass loss rate of the star. Methane is a highly symmetric molecule with no permanent dipole, thus, indirect evidence for the presence of methane can be provided by the search of expected products of $\mathrm{CH}_{4}$ chemistry, such as $\mathrm{C}_{2} \mathrm{H}$ and $\mathrm{CH}_{3} \mathrm{OH}$. A previous search for these molecules toward IK Tau and other O-rich CSEs has resulted in non-detections of $\mathrm{C}_{2} \mathrm{H}\left(f\left(\mathrm{C}_{2} \mathrm{H}\right)<9.7 \times 10^{-9}\right)$ and methanol $\left(f\left(\mathrm{CH}_{3} \mathrm{OH}\right)<\right.$ $3.2 \times 10^{-8}$; Charnley \& Latter 1997; Marvel 2005). We also did not detect emission of these molecules consistent with the upper limits provided by Marvel (2005). If the correct scenario were that $\mathrm{H}_{2} \mathrm{CO}$ is formed in the outer envelope, the size adopted for the calculation of the population diagram would result in an abundance overestimate. In that case, assuming $\theta_{\mathrm{s}}=6^{\prime \prime}$, like for $\mathrm{CN}, \mathrm{NS}$, or NO, we derive an abundance of $f\left(\mathrm{H}_{2} \mathrm{CO}\right) \sim 2 \times 10^{-8}$. Furthermore, a clumpy envelope may lead to an enhanced photochemistry in the inner layers of the CSE, which result in the formation of carbon molecules in the inner and intermediate layers of O-rich CSEs, and in particular formaldehyde with $f\left(\mathrm{H}_{2} \mathrm{CO}\right) \sim 10^{-9}$ (Agúndez et al. 2010).

\subsection{Isotopic ratios}

Isotopic ratios of different species can be measured from the column densities derived in the rotational diagrams (see Table 6). However, these ratios have to be considered as lower limits when the molecule used to calculate the ratio has optically thick lines. In case that the opacities are moderately high, the isotopic ratio can be corrected using the approach by Goldsmith \& Langer (1999).

For ${ }^{12} \mathrm{C} /{ }^{13} \mathrm{C}$ ratio, we measured values of eight to ten depending on the molecule used (i.e. CO, CS or HCN). The opacity correction yielded a ${ }^{12} \mathrm{C} /{ }^{13} \mathrm{C}$ ratio of $\sim 10$ from $\mathrm{CO}$. This value is in good agreement with that obtained by Ramstedt \& Olofsson (2014) from their radiative transfer model of the ${ }^{12} \mathrm{CO}$ and ${ }^{13} \mathrm{CO}$ emission in IK Tau. This ratio is also compatible with other estimates in M-type stars like TX Cam or W Hya, consistent with a standard evolution for an M-type star (Ramstedt \& Olofsson 2014, and references therein).

For the ${ }^{16} \mathrm{O} /{ }^{17} \mathrm{O}$ and ${ }^{16} \mathrm{O} /{ }^{18} \mathrm{O}$ ratios, we estimated lower limits of 90 and 60, respectively. MADEX predicted opacities as high as $\tau \sim 10$ for a few SiO lines that would result in opacity 
corrected values of one order of magnitude higher, in agreement with previous estimates (Decin et al. 2010b).

The opacity corrected isotopic ratios of ${ }^{28} \mathrm{Si} /{ }^{29} \mathrm{Si}$ and ${ }^{28} \mathrm{Si} /{ }^{30} \mathrm{Si}$ are $\sim 18$ and $\sim 34$, respectively. Both isotopic ratios are (within uncertainties) in reasonable agreement with previous estimations toward IK Tau (Decin et al. 2010a), and also with the solar ratios $\left(\left[{ }^{28} \mathrm{Si} /{ }^{29} \mathrm{Si}\right] \sim 20\right.$ and $\left[{ }^{28} \mathrm{Si} /{ }^{30} \mathrm{Si}\right] \sim 30$, Asplund et al. 2009). Therefore, it seems that in the case of IK Tau, Si isotopic ratios do not indicate significant alterations in the post-main sequence evolution.

Finally, we measured the isotopic ratio of ${ }^{32} \mathrm{~S} /{ }^{34} \mathrm{~S}$ using SO, $\mathrm{SiS}, \mathrm{SO}_{2}$, and CS obtaining values between 10 and 13 . We corrected the effect of optically thick emission and we estimated a ${ }^{32} \mathrm{~S} /{ }^{34} \mathrm{~S}$ ratio of $\sim 15$. As far as we know, there are no previous observational constraints to this isotopic ratio toward IK Tau. The solar ${ }^{32} \mathrm{~S} /{ }^{34} \mathrm{~S}$ ratio is $\sim 22$ (Asplund et al. 2009). Recently, Danilovich et al. (2016) reported $\left[{ }^{32} S /{ }^{34} S\right] \sim 32$ toward the Orich CSE of R Dor. Both, Sun and R Dor isotopic ratios are, within uncertainties, compatible with our estimations.

\subsection{Qualitative comparison with other O-rich objects}

The molecular content of only a few O-rich CSEs has been studied so far. In particular, the best studied objects are the AGB CSE IK Tau, the CSE of the hypergiant VY CMa and the peculiar object $\mathrm{OH} 231.8+4.2$ (Alcolea et al. 2013; De Beck et al. 2013; Matsuura et al. 2014; Sánchez Contreras et al. 2015; Ziurys et al. 2007, and references therein).

IK Tau has, in terms of chemical composition, more similarities with VY CMa. $\mathrm{AlOH}$ and $\mathrm{H}_{3} \mathrm{O}^{+}$are the only molecules present in the CSE of VY CMa that are not found in the CSE of IK Tau. Formaldehyde is found in the CSE of IK Tau but it is not found in the CSE of VY CMa. Regardless of the possible chemical processes at work in the CSE of IK Tau, the presence of $\mathrm{CO}, \mathrm{CN}, \mathrm{CS}, \mathrm{HCN}, \mathrm{HNC}, \mathrm{HCO}^{+}$, and $\mathrm{H}_{2} \mathrm{CO}$ in IK Tau indicates that the emission of $\mathrm{C}$-bearing molecules in $\mathrm{VY}$ CMa is not so unique (Ziurys et al. 2009).

$\mathrm{OH} 231.8+4.2$ displays emission of several molecules that are not found toward IK Tau: HNCO, HNCS, OCS, $\mathrm{H}^{13} \mathrm{CO}^{+}, \mathrm{SO}^{+}, \mathrm{N}_{2} \mathrm{H}^{+}$, and $\mathrm{H}_{3} \mathrm{O}^{+}$. The remarkable chemistry of $\mathrm{OH} 231.8+4.2$ probably reflects the molecular regeneration process within its envelope after the passage of fast $\left(\sim 100 \mathrm{~km} \mathrm{~s}^{-1}\right)$ shocks that accelerated and dissociated molecules in the AGB wind $\sim 800 \mathrm{yr}$ ago (Sánchez Contreras et al. 2015). In IK Tau there is no evidence of a similar molecular destruction process by fast $\left(\sim 100 \mathrm{~km} \mathrm{~s}^{-1}\right)$ velocity shocks. Instead, slower shocks due to stellar pulsation may have an impact on the chemistry of AGB CSEs (Gobrecht et al. 2016). However, the fact that these molecules are not observed toward IK Tau point out that slow shocks are not able to enhance the formation of these particular species, which are unexpectedly abundant in $\mathrm{OH} 231.8+4.2$ (Velilla Prieto et al. 2015). Nevertheless, slow shocks could enhance the formation of molecules like HCN or CS (see Sect. 6.3). Another difference with respect to $\mathrm{OH} 231.8+4.2$ is that IK Tau displays emission of $\mathrm{NaCl}$ and more intense lines of vibrationally excited $\mathrm{SiO}$ (Velilla Prieto et al., in prep.). The emission of these lines arises from very warm and inner regions of the $\mathrm{CSE}$. The absence of $\mathrm{NaCl}$ and the weakness of the vibrationally excited $\mathrm{SiO}$ lines toward $\mathrm{OH} 231.8+4.2$, probably indicates that the mass loss rate of $\mathrm{OH} 231.8+4.2$ is decreasing at present (as suggested by Sánchez Contreras et al. 2002), which results in the progressive growth of a central cavity around the star.

\section{Conclusion}

In this work we present the detection toward IK Tau of $~ 350$ rotational lines corresponding to a list of $\mathrm{H}-, \mathrm{O}-, \mathrm{C}-, \mathrm{N}-, \mathrm{S}-, \mathrm{Si}-$ and P-bearing molecules, which evidences an active chemistry for an O-rich AGB CSE.

We detected for first time in this source emission of $\mathrm{HCO}^{+}$, $\mathrm{NO}, \mathrm{H}_{2} \mathrm{CO}$, and NS. We also detected for the first time toward IK Tau rotational lines of $\mathrm{C}^{18} \mathrm{O}, \mathrm{Si}^{17} \mathrm{O}, \mathrm{Si}^{18} \mathrm{O},{ }^{29} \mathrm{SiS},{ }^{30} \mathrm{SiS}$, $\mathrm{Si}^{34} \mathrm{~S}, \mathrm{H}^{13} \mathrm{CN},{ }^{13} \mathrm{CS}, \mathrm{C}^{34} \mathrm{~S}, \mathrm{H}_{2}{ }^{34} \mathrm{~S},{ }^{34} \mathrm{SO},{ }^{34} \mathrm{SO}_{2}$, and $\mathrm{H}_{2} \mathrm{O} v_{2}=2$, as well as several rotational lines of $\mathrm{SiO}$ isotopologues in vibrationally excited states. In addition, we significantly increased the number of lines detected for those molecules that were previously identified toward IK Tau. This has allowed us to deduce characteristic values of the rotational temperatures, column densities, and ultimately fractional abundances of the molecules present in its envelope. From our work we extract the following conclusions:

- The intensity of the rotational lines of molecules in the ground vibrational state do not show a significant variability as a function of time, for the spectral ranges that we could observe in different epochs. The small variations found for these lines can be explained owing to calibration or pointing uncertainties within a a $25 \%$. We confirmed the time variability of the intensity of the lines of molecules in vibrationally excited states (e.g. $\mathrm{SiO} v=1$ ) by an average factor of $60 \%$.

- Most of the molecules display rotational temperatures between 15 and $40 \mathrm{~K}$. $\mathrm{NaCl}$ and $\mathrm{SiS}$ isotopologues display rotational temperatures of $\sim 65 \mathrm{~K}$.

- We detected a warm component of $\mathrm{SO}_{2}$ traced out by lines with upper energy levels between 160 and $730 \mathrm{~K}$ which display $v_{\exp }<v_{\infty}$. This points out that $\mathrm{SO}_{2}$ is present close to the stellar surface $\left(\lesssim 8 R_{*}\right)$ with an abundance of $f\left(\mathrm{SO}_{2}\right) \sim 10^{-6}$.

- Among the species detected, we highlight the detection of $\mathrm{H}_{2} \mathrm{CO}$ and NS for the first time in this source with abundances of $f\left(\mathrm{H}_{2} \mathrm{CO}\right) \sim\left[10^{-7}-10^{-8}\right]$ and $f(\mathrm{NS}) \sim 10^{-8}$. We also estimated fractional abundances for the first time detected (toward IK Tau) molecules $\mathrm{HCO}^{+}$and $\mathrm{NO}$ obtaining $f\left(\mathrm{HCO}^{+}\right) \sim 10^{-8}$ and $f(\mathrm{NO}) \sim 10^{-6}$.

- The detection of several C-bearing species like $\mathrm{HCN}, \mathrm{CS}$, $\mathrm{H}_{2} \mathrm{CO}$ or $\mathrm{CN}$ with abundances of $\sim 10^{-7}$ indicates an active carbon chemistry which is not expected given that most of the available carbon should be locked up into $\mathrm{CO}$.

- The greatest discrepancies between our results and previous chemical models are found for $\mathrm{PO}, \mathrm{NaCl}$, and $\mathrm{SO}_{2}$.

It would be necessary to obtain very high angular resolution observations to characterise the molecular emission in the inner parts of the CSE and the abundances and distribution of the molecules formed in this region. Further investigation is required to understand the nature of the discrepancies found between our derived values and chemical models, in particular, the discrepancies for S-bearing molecules and C-bearing molecules. The inclusion of photo-induced or shock-induced chemistry or maybe other processes is necessary to enhance the formation of these molecules up to values comparable to the abundances observed. Additionally, there are $\sim 40$ lines that still remain unidentified. We expect that future observations, supported by improvements in the molecular catalogues and chemical models, lead to fully understand the envelope of IK Tau and, more generally, in O-rich AGB envelopes.

Acknowledgements. We acknowledge the IRAM staff for the support and help offered during all the observational runs. We acknowledge the Spanish MICINN/MINECO for funding support through grants AYA2009-07304, 
AYA2012-32032, the ASTROMOL Consolider project CSD2009-00038 and also the European Research Council funding support (ERC grant 610256: NANOCOSMOS). L.V.P. also acknowledges the support of the Universidad Complutense de Madrid Ph.D. programme. This research has made use of the The JPL Molecular Spectroscopy catalog, The Cologne Database for Molecular Spectroscopy, the SIMBAD database operated at CDS (Strasbourg, France), the NASA's Astrophysics Data System, the IRAM GILDAS software, and Aladin.

\section{References}

Alcolea, J., \& Bujarrabal, V. 1992, A\&A, 253, 475

Alcolea, J., Pardo, J. R., Bujarrabal, V., et al. 1999, A\&AS, 139, 461

Alcolea, J., Bujarrabal, V., Planesas, P., et al. 2013, A\&A, 559, A93

Asplund, M., Grevesse, N., Sauval, A. J., \& Scott, P. 2009, ARA\&A, 47, 481

Agúndez, M., \& Cernicharo, J. 2006, ApJ, 650, 374

Agúndez, M., Cernicharo, J., \& Guélin, M. 2007, ApJ, 662, L91

Agúndez, M., Cernicharo, J., \& Guélin, M. 2010, ApJ, 724, L133

Agúndez, M., Fonfría, J. P., Cernicharo, J., et al. 2012, A\&A, 543, A48

Ben Abdallah, D., Najar, F., Jaidane, N., Dumouchel, F., \& Lique, F. 2012 MNRAS, 419, 2441

Bieging, J. H., \& Tafalla, M. 1993, AJ, 105, 576

Bowers, P. F., Johnston, K. J., \& de Vegt, C. 1989, ApJ, 340, 479

Bujarrabal, V., \& Alcolea, J. 1991, A\&A, 251, 536

Bujarrabal, V., Fuente, A., \& Omont, A. 1994, A\&A, 285, 247

Carter, M., Lazareff, B., Maier, D., et al. 2012, A\&A, 538, A89

Castro-Carrizo, A., Quintana-Lacaci, G., Neri, R., et al. 2010, A\&A, 523, A59

Cernicharo, J. 1985, Internal IRAM report (Granada: IRAM)

Cernicharo, J. 2012, EAS Pub. Ser., 58, 251

Cernicharo, J., Guélin, M., \& Kahane, C. 2000, A\&AS, 142, 181

Cernicharo, J., Spielfiedel, A., Balança, C., et al. 2011, A\&A, 531, A103

Cernicharo, J., Teyssier, D., Quintana-Lacaci, G., et al. 2014, ApJ, 796, L21

Cernicharo, J., McCarthy, M. C., Gottlieb, C. A., et al. 2015, ApJ, 806, L3

Charnley, S. B., \& Latter, W. B. 1997, MNRAS, 287, 538

Charnley, S. B., Tielens, A. G. G. M., \& Kress, M. E. 1995, MNRAS, 274, L53

Cherchneff, I. 2006, A\&A, 456, 1001

Cutri, R. M., Skrutskie, M. F., van Dyk, S., et al. 2003, VizieR Online Data Catalog: II/246

Daniel, F., Dubernet, M.-L., \& Grosjean, A. 2011, A\&A, 536, A76

Danilovich, T., De Beck, E., Black, J. H., Olofsson, H., \& Justtanont, K. 2016, A\&A, 588, A119

Dayou, F., \& Balança, C. 2006, A\&A, 459, 297

De Beck, E., Kamiński, T., Patel, N. A., et al. 2013, A\&A, 558, A132

De Beck, E., Kamiński, T., Menten, K. M., et al. 2015, Why Galaxies Care about AGB Stars III: A Closer Look in Space and Time, 497, 73

Decin, L., Hony, S., de Koter, A., et al. 2006, A\&A, 456, 549

Decin, L., De Beck, E., Brünken, S., et al. 2010a, A\&A, 516, A69

Decin, L., Justtanont, K., De Beck, E., et al. 2010b, A\&A, 521, L4

Duari, D., Cherchneff, I., \& Willacy, K. 1999, A\&A, 341, L47

Flower, D. R. 1999, MNRAS, 305, 651

Gobrecht, D., Cherchneff, I., Sarangi, A., Plane, J. M. C., \& Bromley, S. T. 2016, A\&A, 585, A6

Goldreich, P., \& Kwan, J. 1974, ApJ, 189, 441

Goldsmith, P. F., \& Langer, W. D. 1999, ApJ, 517, 209

González Delgado, D., Olofsson, H., Kerschbaum, F., et al. 2003, A\&A, 411, 123

Green, S. 1976, J. Chem. Phys., 64, 3463

Green, S. 1979, J. Chem. Phys., 70, 816

Habing, H.J., \& Olofsson, H. 2004, AGB Stars, A\&A Library (Berlin: Springer)

Hale, D. D. S., Bester, M., Danchi, W. C., et al. 1997, ApJ, 490, 407

Justtanont, K., Khouri, T., Maercker, M., et al. 2012, A\&A, 537, A144

Kim, H., Wyrowski, F., Menten, K. M., \& Decin, L. 2010, A\&A, 516, A68

Lane, A. P., Johnston, K. J., Bowers, P. F., Spencer, J. H., \& Diamond, P. J. 1987, ApJ, 323, 756

Li, X., Millar, T. J., Heays, A. N., et al. 2016, A\&A, 588, A4

Lindqvist, M., Nyman, L.-A., Olofsson, H., \& Winnberg, A. 1988, A\&A, 205, L15

Lindqvist, M., Olofsson, H., Winnberg, A., \& Nyman, L. A. 1992, A\&A, 263, 183
Lique, F., \& Kłos, J. 2011, MNRAS, 413, L20

Lique, F., \& Spielfiedel, A. 2007, A\&A, 462, 1179

Lique, F., Senent, M.-L., Spielfiedel, A., \& Feautrier, N. 2007, J. Chem. Phys., 126,164312

Lucas, R., Bujarrabal, V., Guilloteau, S., et al. 1992, A\&A, 262, 491

Lucas, R., Guélin, M., Kahane, C., Audinos, P., \& Cernicharo, J. 1995, Ap\&SS, 224,293

Marvel, K. B. 2005, AJ, 130, 261

Matsuura, M., Yates, J. A., Barlow, M. J., et al. 2014, MNRAS, 437, 532

Menten, K. M., Wyrowski, F., Alcolea, J., et al. 2010, A\&A, 521, L7

Milam, S. N., Apponi, A. J., Woolf, N. J., \& Ziurys, L. M. 2007, ApJ, 668, L131

Milam, S. N., Halfen, D. T., Tenenbaum, E. D., et al. 2008, ApJ, 684, 618-625

Millar, T. J., \& Olofsson, H. 1993, MNRAS, 262, L55

Morris, M., Gilmore, W., Palmer, P., Turner, B. E., \& Zuckerman, B. 1975, ApJ, 199, L47

Müller, H. S. P., Schlöder, F., Stutzki, J., \& Winnewisser, G. 2005, J. Mol. Struct., 742,215

Nakashima, J.-I., \& Deguchi, S. 2007, ApJ, 669, 446

Nejad, L. A. M., \& Millar, T. J. 1988, MNRAS, 230, 79

Nercessian, E., Omont, A., Benayoun, J. J., \& Guilloteau, S. 1989, A\&A, 210, 225

Neri, R., Kahane, C., Lucas, R., Bujarrabal, V., \& Loup, C. 1998, A\&AS, 130, 1

Neugebauer, G., Martz, D. E., \& Leighton, R. B. 1965, ApJ, 142, 399

Olofsson, H. 1996, Ap\&SS, 245, 169

Olofsson, H., Lindqvist, M., Nyman, L.-A., \& Winnberg, A. 1998, A\&A, 329, 1059

Omont, A., Lucas, R., Morris, M., \& Guilloteau, S. 1993, A\&A, 267, 490

Pardo, J. R., Cernicharo, J., \& Serabyn, E. 2001, IEEE Trans. Antennas and Propagation, 49, 1683

Pesch, P. 1967, ApJ, 147, 381

Phillips, T. G., Huggins, P. J., Wannier, P. G., \& Scoville, N. Z. 1979, ApJ, 231, 720

Pickett, H. M., Poynter, R. L., Cohen, E. A., et al. 1998, J. Quant. Spectr. Rad. Trans. 60, 883

Quintana-Lacaci, G., Cernicharo, J., Agúndez, M., et al. 2016, ApJ, 818, 192

Ramstedt, S., \& Olofsson, H. 2014, A\&A, 566, A145

Sánchez Contreras, C., Desmurs, J. F., Bujarrabal, V., Alcolea, J., \& Colomer, F. 2002, A\&A, 385, L1

Sánchez Contreras, C., Velilla, L., Alcolea, J., et al. 2014, Asymmetrical Planetary Nebulae VI Conference, 88

Sánchez Contreras, C., Velilla Prieto, L., Agúndez, M., et al. 2015, A\&A, 577, A52

Schöier, F. L., van der Tak, F. F. S., van Dishoeck, E. F., \& Black, J. H. 2005, A\&A, 432, 369

Schöier, F. L., Ramstedt, S., Olofsson, H., et al. 2013, A\&A, 550, A78

Smith, C. L., Zijlstra, A. A., \& Fuller, G. A. 2015, MNRAS, 454, 177

Solomon, P., Jefferts, K. B., Penzias, A. A., \& Wilson, R. W. 1971, ApJ, 163, L53

Spielfiedel, A., Senent, M.-L., Dayou, F., et al. 2009, J. Chem. Phys., 131, 014305

Suh, K.-W. 1999, MNRAS, 304, 389

Toboła, R., Kłos, J., Lique, F., Chałasiński, G., \& Alexander, M. H. 2007, A\&A, 468, 1123

Toboła, R., Lique, F., Kłos, J., \& Chałasiński, G. 2008, J. Phys. B At. Mol. Phys., 41, 155702

Tsuji, T. 1973, A\&A, 23, 411

Velilla Prieto, L., Sánchez Contreras, C., Cernicharo, J., et al. 2013, Highlights of Spanish Astrophysics VII, 676

Velilla Prieto, L., Sánchez Contreras, C., Cernicharo, J., et al. 2015, A\&A, 575, A84

Willacy, K., \& Millar, T. J. 1997, A\&A, 324, 237

Wing, R. F., \& Lockwood, G. W. 1973, ApJ, 184, 873

Yang, B., Stancil, P. C., Balakrishnan, N., \& Forrey, R. C. 2010, ApJ, 718, 1062

Zhang, Y., Kwok, S., \& Nakashima, J.-I. 2009, ApJ, 700, 1262

Ziurys, L. M., Milam, S. N., Apponi, A. J., \& Woolf, N. J. 2007, Nature, 447, 1094

Ziurys, L. M., Tenenbaum, E. D., Pulliam, R. L., Woolf, N. J., \& Milam, S. N. 2009, ApJ, 695, 1604

Zuckerman, B. 1987, Astrochemistry, 120, 345 


\section{Appendix A: Table of measured lines}

Table A.1. Measured and spectroscopic parameters of the detected lines.

\begin{tabular}{|c|c|c|c|c|c|c|}
\hline Molecule & $\begin{array}{l}\text { Transition } \\
\text { QNs }\end{array}$ & $\begin{array}{l}v_{\text {rest }} \\
(\mathrm{MHz})\end{array}$ & $\begin{array}{l}E_{\mathrm{u}} \\
(\mathrm{K})\end{array}$ & $\begin{array}{l}A_{\mathrm{ul}} \\
\left(\mathrm{s}^{-1}\right)\end{array}$ & $\begin{array}{l}\int T_{\text {ant }}^{*} \mathrm{~d} v \\
\left(\mathrm{~K} \mathrm{~km} \mathrm{~s}^{-1}\right)\end{array}$ & $\begin{array}{l}v_{\exp } \\
\left(\mathrm{km} \mathrm{s}^{-1}\right)\end{array}$ \\
\hline $\mathrm{CO}$ & $1-0$ & 115271.202 & 5.5 & $7.205 \times 10^{-8}$ & $49.14(0.04)$ & $18(3)$ \\
\hline $\mathrm{CO}$ & $2-1$ & 230538.000 & 16.6 & $6.916 \times 10^{-7}$ & $121.61(0.06)$ & $16.7(1.3)$ \\
\hline $\mathrm{CO}$ & $3-2$ & 345795.990 & 33.2 & $2.501 \times 10^{-6}$ & $103.38(0.07)$ & $16.7(1.0)$ \\
\hline${ }^{13} \mathrm{CO}$ & $1-0$ & 110201.354 & 5.3 & $6.336 \times 10^{-8}$ & $5.08(0.02)$ & $19(3)$ \\
\hline${ }^{13} \mathrm{CO}$ & $2-1$ & 220398.684 & 15.9 & $6.082 \times 10^{-7}$ & $13.11(0.05)$ & $18.6(1.4)$ \\
\hline${ }^{13} \mathrm{CO}$ & $3-2$ & 330587.964 & 31.7 & $2.199 \times 10^{-6}$ & $15.37(0.12)$ & $18.1(1.0)$ \\
\hline $\mathrm{C}^{18} \mathrm{O}$ & $1-0$ & 109782.176 & 5.3 & $6.263 \times 10^{-8}$ & $0.07(0.02)$ & $14(6)$ \\
\hline $\mathrm{C}^{18} \mathrm{O}$ & $2-1$ & 219560.358 & 15.8 & $6.013 \times 10^{-7}$ & $0.39(0.03)$ & $19.8(1.4)$ \\
\hline $\mathrm{SiO}$ & $2-1$ & 86846.986 & 6.3 & $2.927 \times 10^{-5}$ & $31.16(0.03)$ & $17(3)$ \\
\hline $\mathrm{SiO}$ & $3-2$ & 130268.687 & 12.5 & $1.059 \times 10^{-4}$ & $50.51(0.03)$ & $18(2)$ \\
\hline $\mathrm{SiO}$ & $4-3$ & 173688.237 & 20.8 & $2.602 \times 10^{-4}$ & $48.63(0.06)$ & $18(2)$ \\
\hline $\mathrm{SiO}$ & $5-4$ & 217104.920 & 31.3 & $5.197 \times 10^{-4}$ & $59.86(0.04)$ & $17.0(1.4)$ \\
\hline $\mathrm{SiO}$ & $6-5$ & 260518.018 & 43.8 & $9.117 \times 10^{-4}$ & $56.51(0.07)$ & $14.4(1.2)$ \\
\hline $\mathrm{SiO}$ & $7-6$ & 303926.814 & 58.4 & $1.464 \times 10^{-3}$ & $56.96(0.06)$ & $17.6(1.0)$ \\
\hline $\mathrm{SiO}$ & $8-7$ & 347330.592 & 75.0 & $2.203 \times 10^{-3}$ & $40.76(0.15)$ & $17.3(1.0)$ \\
\hline${ }^{29} \mathrm{SiO}$ & $2-1$ & 85759.194 & 6.2 & $2.819 \times 10^{-5}$ & $5.85(0.03)$ & $17(3)$ \\
\hline${ }^{29} \mathrm{SiO}$ & $3-2$ & 128637.044 & 12.3 & $1.019 \times 10^{-4}$ & $10.71(0.03)$ & $15(2)$ \\
\hline${ }^{29} \mathrm{SiO}$ & $4-3$ & 171512.796 & 20.6 & $2.505 \times 10^{-4}$ & $13.05(0.05)$ & $18(2)$ \\
\hline${ }^{29} \mathrm{SiO}$ & $5-4$ & 214385.752 & 30.9 & $5.004 \times 10^{-4}$ & $14.82(0.04)$ & $17.8(1.4)$ \\
\hline${ }^{29} \mathrm{SiO}$ & $6-5$ & 257255.213 & 43.2 & $8.779 \times 10^{-4}$ & $12.88(0.05)$ & $17.9(1.2)$ \\
\hline${ }^{29} \mathrm{SiO}$ & $7-6$ & 300120.480 & 57.6 & $1.409 \times 10^{-3}$ & $10.60(0.08)$ & $17.1(1.0)$ \\
\hline${ }^{29} \mathrm{SiO}$ & $8-7$ & 342980.854 & 74.1 & $2.121 \times 10^{-3}$ & $10.27(0.09)$ & $16.7(1.0)$ \\
\hline${ }^{30} \mathrm{SiO}$ & $2-1$ & 84746.165 & 6.1 & $2.720 \times 10^{-5}$ & $3.74(0.03)$ & $18(3)$ \\
\hline${ }^{30} \mathrm{SiO}$ & $4-3$ & 169486.869 & 20.3 & $2.418 \times 10^{-4}$ & $8.72(0.03)$ & $19(2)$ \\
\hline${ }^{30} \mathrm{SiO}$ & $5-4$ & 211853.467 & 30.5 & $4.829 \times 10^{-4}$ & $11.78(0.06)$ & $16.1(1.4)$ \\
\hline${ }^{30} \mathrm{SiO}$ & $6-5$ & 254216.652 & 42.7 & $8.472 \times 10^{-4}$ & $8.96(0.03)$ & $17.0(1.2)$ \\
\hline${ }^{30} \mathrm{SiO}$ & $7-6$ & 296575.741 & 56.9 & $1.360 \times 10^{-3}$ & $10.39(0.03)$ & $20.0(1.0)$ \\
\hline${ }^{30} \mathrm{SiO}$ & $8-7$ & 338930.052 & 73.2 & $2.047 \times 10^{-3}$ & $6.78(0.15)$ & $16.8(1.1)$ \\
\hline $\mathrm{Si}^{18} \mathrm{O}$ & $2-1$ & 80704.922 & 5.8 & $2.349 \times 10^{-5}$ & $0.21(0.03)$ & $19(4)$ \\
\hline $\mathrm{Si}^{18} \mathrm{O}$ & $4-3$ & 161404.893 & 19.4 & $2.088 \times 10^{-4}$ & $0.82(0.02)$ & $16(2)$ \\
\hline $\mathrm{Si}^{18} \mathrm{O}$ & $6-5$ & 242094.961 & 40.7 & $7.317 \times 10^{-4}$ & $1.34(0.05)$ & $16.9(1.3)$ \\
\hline $\mathrm{Si}^{18} \mathrm{O}$ & $7-6$ & 282434.735 & 54.2 & $1.175 \times 10^{-3}$ & $2.56(0.04)$ & $16.8(1.1)$ \\
\hline $\mathrm{Si}^{17} \mathrm{O}$ & $4-3$ & 167171.991 & 20.1 & $2.320 \times 10^{-4}$ & $0.89(0.02)$ & $11(3)$ \\
\hline $\mathrm{Si}^{17} \mathrm{O}$ & $5-4$ & 208960.009 & 30.1 & $4.634 \times 10^{-4}$ & $0.52(0.04)$ & $21.9(1.4)$ \\
\hline $\mathrm{Si}^{17} \mathrm{O}$ & $6-5$ & 250744.706 & 42.1 & $8.129 \times 10^{-4}$ & $0.43(0.05)$ & $18(3)$ \\
\hline $\mathrm{Si}^{17} \mathrm{O}$ & $7-6$ & 292525.420 & 56.2 & $1.305 \times 10^{-3}$ & $0.27(0.04)$ & $9(2)$ \\
\hline $\mathrm{SiS}$ & $5-4$ & 90771.566 & 13.1 & $1.191 \times 10^{-5}$ & $1.02(0.02)$ & $20(3)$ \\
\hline $\mathrm{SiS}$ & $6-5$ & 108924.303 & 18.3 & $2.090 \times 10^{-5}$ & $2.04(0.03)$ & $18(3)$ \\
\hline $\mathrm{SiS}$ & $8-7$ & 145227.054 & 31.4 & $5.050 \times 10^{-5}$ & $4.23(0.02)$ & $18(2)$ \\
\hline $\mathrm{SiS}$ & $9-8$ & 163376.782 & 39.2 & $7.238 \times 10^{-5}$ & $5.88(0.02)$ & $19(2)$ \\
\hline $\mathrm{SiS}$ & $12-11$ & 217817.656 & 68.0 & $1.738 \times 10^{-4}$ & $9.36(0.05)$ & $19.2(1.4)$ \\
\hline $\mathrm{SiS}$ & $13-12$ & 235961.366 & 79.3 & $2.216 \times 10^{-4}$ & $11.13(0.06)$ & $19.1(1.3)$ \\
\hline $\mathrm{SiS}$ & $14-13$ & 254103.213 & 91.5 & $2.775 \times 10^{-4}$ & $11.68(0.04)$ & $18.8(1.2)$ \\
\hline $\mathrm{SiS}$ & $15-14$ & 272243.055 & 104.5 & $3.421 \times 10^{-4}$ & $7.50(0.07)$ & $19.0(1.1)$ \\
\hline $\mathrm{SiS}$ & $16-15$ & 290380.747 & 118.5 & $4.159 \times 10^{-4}$ & $13.18(0.04)$ & $18.6(1.0)$ \\
\hline $\mathrm{SiS}$ & $17-16$ & 308516.147 & 133.3 & $4.997 \times 10^{-4}$ & $10.28(0.05)$ & $17.9(1.0)$ \\
\hline $\mathrm{SiS}$ & $19-18$ & 344779.495 & 165.5 & $6.996 \times 10^{-4}$ & $11.87(0.10)$ & $17.3(1.0)$ \\
\hline
\end{tabular}

Notes. Column 2: quantum numbers; Col. 6: integrated intensity (in antenna temperature) of the line with its formal uncertainty; Col. 7: expansion velocity derived from the linewidth with its uncertainty which includes the width of half a channel (1 MHz) except for the lines with a low S/N where a full channel $(2 \mathrm{MHz})$ is included. The uncertainties given (within parentheses) do not include additional uncertainties derived from the absolute flux calibration. (HB) Hard blend, thus, we cannot estimate the contribution of the line to the spectral feature observed. (BF) Bad fit. 
Table A.1. continued.

\begin{tabular}{|c|c|c|c|c|c|c|}
\hline Molecule & $\begin{array}{l}\text { Transition } \\
\text { QNs }\end{array}$ & $\begin{array}{l}v_{\text {rest }} \\
(\mathrm{MHz})\end{array}$ & $\begin{array}{l}E_{\mathrm{u}} \\
(\mathrm{K})\end{array}$ & $\begin{array}{l}A_{\mathrm{ul}} \\
\left(\mathrm{s}^{-1}\right) \\
\end{array}$ & $\begin{array}{l}\int T_{\text {ant }}^{*} \mathrm{~d} v \\
\left(\mathrm{~K} \mathrm{~km} \mathrm{~s}^{-1}\right)\end{array}$ & $\begin{array}{l}v_{\exp } \\
\left(\mathrm{km} \mathrm{s}^{-1}\right)\end{array}$ \\
\hline${ }^{29} \mathrm{SiS}$ & $6-5$ & 106922.982 & 18.0 & $1.977 \times 10^{-5}$ & $0.14(0.02)$ & $17(4)$ \\
\hline${ }^{29} \mathrm{SiS}$ & $8-7$ & 142558.821 & 30.8 & $4.777 \times 10^{-5}$ & $0.29(0.03)$ & $18(3)$ \\
\hline${ }^{29} \mathrm{SiS}$ & $9-8$ & 160375.153 & 38.5 & $6.846 \times 10^{-5}$ & $0.50(0.02)$ & $17(2)$ \\
\hline${ }^{29} \mathrm{SiS}$ & $12-11$ & 213816.142 & 66.7 & $1.644 \times 10^{-4}$ & $1.02(0.04)$ & $18(2)$ \\
\hline${ }^{29} \mathrm{SiS}$ & $13-12$ & 231626.676 & 77.8 & $2.096 \times 10^{-4}$ & $1.22(0.06)$ & $18.8(1.5)$ \\
\hline${ }^{29} \mathrm{SiS}$ & $14-13$ & 249435.415 & 89.8 & $2.625 \times 10^{-4}$ & $1.10(0.05)$ & $17.6(1.3)$ \\
\hline${ }^{29} \mathrm{SiS}$ & $15-14$ & 267242.221 & 102.6 & $3.236 \times 10^{-4}$ & $1.15(0.05)$ & $16(2)$ \\
\hline${ }^{29} \mathrm{SiS}$ & $16-15$ & 285046.956 & 116.3 & $3.934 \times 10^{-4}$ & $1.28(0.06)$ & $14.5(1.2)$ \\
\hline${ }^{29} \mathrm{SiS}$ & $17-16$ & 302849.482 & 130.8 & $4.727 \times 10^{-4}$ & $1.23(0.03)$ & $17.7(1.1)$ \\
\hline${ }^{29} \mathrm{SiS}$ & $18-17$ & 320649.661 & 146.2 & $5.619 \times 10^{-4}$ & $0.99(0.11)$ & $15(2)$ \\
\hline${ }^{29} \mathrm{SiS}$ & $19-18$ & 338447.355 & 162.5 & $6.617 \times 10^{-4}$ & $1.17(0.12)$ & $16(2)$ \\
\hline${ }^{30} \mathrm{SiS}$ & $8-7$ & 140073.964 & 30.3 & $4.532 \times 10^{-5}$ & $0.26(0.02)$ & $20(2)$ \\
\hline${ }^{30} \mathrm{SiS}$ & $9-8$ & 157579.811 & 37.8 & $6.494 \times 10^{-5}$ & $0.27(0.02)$ & $19(2)$ \\
\hline${ }^{30} \mathrm{SiS}$ & $12-11$ & 210089.621 & 65.5 & $1.560 \times 10^{-4}$ & $0.67(0.05)$ & $12(2)$ \\
\hline${ }^{30} \mathrm{SiS}$ & $13-12$ & 227589.870 & 76.5 & $1.989 \times 10^{-4}$ & $0.62(0.04)$ & $17(2)$ \\
\hline${ }^{30} \mathrm{SiS}$ & $14-13$ & 245088.386 & 88.2 & $2.490 \times 10^{-4}$ & $0.67(0.04)$ & $16.7(1.5)$ \\
\hline${ }^{30} \mathrm{SiS}$ & $15-14$ & 262585.036 & 100.8 & $3.070 \times 10^{-4}$ & $0.86(0.06)$ & $17.6(1.2)$ \\
\hline${ }^{30} \mathrm{SiS}$ & $16-15$ & 280079.687 & 114.3 & $3.732 \times 10^{-4}$ & $1.17(0.04)$ & $20.3(1.3)$ \\
\hline${ }^{30} \mathrm{SiS}$ & $17-16$ & 297572.206 & 128.6 & $4.484 \times 10^{-4}$ & $0.88(0.05)$ & $13.6(1.0)$ \\
\hline${ }^{30} \mathrm{SiS}$ & $18-17$ & 315062.459 & 143.7 & $5.331 \times 10^{-4}$ & $0.71(0.06)$ & $17(2)$ \\
\hline${ }^{30} \mathrm{SiS}$ & 19-18 & 332550.312 & 159.6 & $6.278 \times 10^{-4}$ & $0.85(0.09)$ & $15(2)$ \\
\hline $\mathrm{Si}^{34} \mathrm{~S}$ & $5-4$ & 88285.830 & 12.7 & $1.096 \times 10^{-5}$ & $0.09(0.02)$ & $7(7)$ \\
\hline $\mathrm{Si}^{34} \mathrm{~S}$ & $6-5$ & 105941.505 & 17.8 & $1.923 \times 10^{-5}$ & $0.18(0.02)$ & $24(5)$ \\
\hline $\mathrm{Si}^{34} \mathrm{~S}$ & $8-7$ & 141250.280 & 30.5 & $4.647 \times 10^{-5}$ & $0.18(0.02)$ & $15(2)$ \\
\hline $\mathrm{Si}^{34} \mathrm{~S}$ & $9-8$ & 158903.109 & 38.1 & $6.659 \times 10^{-5}$ & $0.44(0.02)$ & $20(2)$ \\
\hline $\mathrm{Si}^{34} \mathrm{~S}$ & $13-12$ & 229500.871 & 77.1 & $2.039 \times 10^{-4}$ & $0.96(0.05)$ & $15.2(1.5)$ \\
\hline $\mathrm{Si}^{34} \mathrm{~S}$ & $14-13$ & 247146.245 & 89.0 & $2.553 \times 10^{-4}$ & $0.96(0.03)$ & $17.1(1.3)$ \\
\hline $\mathrm{Si}^{34} \mathrm{~S}$ & $15-14$ & 264789.722 & 101.7 & $3.147 \times 10^{-4}$ & $0.97(0.05)$ & $17.2(1.3)$ \\
\hline $\mathrm{Si}^{34} \mathrm{~S}$ & $17-16$ & 300070.442 & 129.6 & $4.598 \times 10^{-4}$ & $1.01(0.11)$ & $16(2)$ \\
\hline $\mathrm{Si}^{34} \mathrm{~S}$ & $18-17$ & 317707.414 & 144.9 & $5.466 \times 10^{-4}$ & $1.07(0.13)$ & $13(2)$ \\
\hline $\mathrm{HCO}^{+}$ & $1-0$ & 89188.526 & 4.3 & $4.234 \times 10^{-5}$ & $0.45(0.03)$ & $20(3)$ \\
\hline $\mathrm{HNC}$ & $1-0$ & 90663.563 & 4.4 & $2.690 \times 10^{-5}$ & $0.13(0.02)$ & $20(7)$ \\
\hline HNC & $3-2$ & 271981.107 & 26.1 & $9.336 \times 10^{-4}$ & $\mathrm{HB}$ & $\mathrm{HB}$ \\
\hline $\mathrm{HCN}$ & $1-0$ & 88631.602 & 4.3 & $2.406 \times 10^{-5}$ & $8.09(0.02)$ & $18(3)$ \\
\hline $\mathrm{HCN}$ & $3-2$ & 265886.433 & 25.5 & $8.352 \times 10^{-4}$ & $33.02(0.04)$ & $18.2(1.1)$ \\
\hline $\mathrm{HCN}$ & $4-3$ & 354505.476 & 42.5 & $2.053 \times 10^{-3}$ & $20.5(0.2)$ & $17.6(1.0)$ \\
\hline $\mathrm{H}^{13} \mathrm{CN}$ & $1-0$ & 86339.921 & 4.1 & $2.224 \times 10^{-5}$ & $0.85(0.04)$ & $18(4)$ \\
\hline $\mathrm{H}^{13} \mathrm{CN}$ & $2-1$ & 172677.851 & 12.4 & $2.135 \times 10^{-4}$ & $3.90(0.09)$ & $16(2)$ \\
\hline $\mathrm{H}^{13} \mathrm{CN}$ & $3-2$ & 259011.798 & 24.9 & $7.721 \times 10^{-4}$ & $6.43(0.05)$ & $16.4(1.2)$ \\
\hline $\mathrm{H}^{13} \mathrm{CN}$ & $4-3$ & 345339.769 & 41.4 & $1.898 \times 10^{-3}$ & HB & $\mathrm{HB}$ \\
\hline $\mathrm{NaCl}$ & $8-7$ & 104189.669 & 22.5 & $5.020 \times 10^{-4}$ & $0.05(0.01)$ & $10(8)$ \\
\hline $\mathrm{NaCl}$ & $10-9$ & 130223.637 & 34.4 & $9.919 \times 10^{-4}$ & $0.10(0.01)$ & $15(5)$ \\
\hline $\mathrm{NaCl}$ & $11-10$ & 143237.371 & 41.3 & $1.326 \times 10^{-3}$ & $0.18(0.02)$ & $20(4)$ \\
\hline $\mathrm{NaCl}$ & $12-11$ & 156248.639 & 48.8 & $1.727 \times 10^{-3}$ & $0.21(0.02)$ & $14(4)$ \\
\hline $\mathrm{NaCl}$ & $13-12$ & 169257.217 & 56.9 & $2.202 \times 10^{-3}$ & $0.27(0.02)$ & $16(2)$ \\
\hline $\mathrm{NaCl}$ & $16-15$ & 208264.570 & 85.0 & $4.131 \times 10^{-3}$ & $0.15(0.02)$ & $8(3)$ \\
\hline $\mathrm{NaCl}$ & $17-16$ & 221260.147 & 95.6 & $4.962 \times 10^{-3}$ & $0.24(0.03)$ & $10(4)$ \\
\hline $\mathrm{NaCl}$ & $18-17$ & 234251.912 & 106.9 & $5.898 \times 10^{-3}$ & $0.27(0.04)$ & $11(3)$ \\
\hline $\mathrm{NaCl}$ & $19-18$ & 247239.643 & 118.7 & $6.945 \times 10^{-3}$ & $0.40(0.03)$ & $17(2)$ \\
\hline $\mathrm{NaCl}$ & $20-19$ & 260223.113 & 131.2 & $8.108 \times 10^{-3}$ & $0.27(0.06)$ & $11(3)$ \\
\hline $\mathrm{NaCl}$ & $22-21$ & 286176.379 & 158.1 & $1.081 \times 10^{-2}$ & $0.28(0.05)$ & $20(3)$ \\
\hline $\mathrm{NaCl}$ & $23-22$ & 299145.726 & 172.4 & $1.236 \times 10^{-2}$ & $0.25(0.02)$ & $13.8(1.5)$ \\
\hline $\mathrm{NaCl}$ & $24-23$ & 312109.915 & 187.4 & $1.405 \times 10^{-2}$ & $0.28(0.06)$ & $14(2)$ \\
\hline
\end{tabular}


Table A.1. continued.

\begin{tabular}{|c|c|c|c|c|c|c|}
\hline Molecule & $\begin{array}{l}\text { Transition } \\
\text { QNs }\end{array}$ & $\begin{array}{l}v_{\text {rest }} \\
(\mathrm{MHz})\end{array}$ & $\begin{array}{l}E_{\mathrm{u}} \\
(\mathrm{K})\end{array}$ & $\begin{array}{l}A_{\mathrm{ul}} \\
\left(\mathrm{s}^{-1}\right)\end{array}$ & $\begin{array}{l}\int T_{\mathrm{ant}}^{*} \mathrm{~d} v \\
\left(\mathrm{~K} \mathrm{~km} \mathrm{~s}^{-1}\right)\end{array}$ & $\begin{array}{l}v_{\exp } \\
\left(\mathrm{km} \mathrm{s}^{-1}\right)\end{array}$ \\
\hline $\mathrm{CS}$ & $2-1$ & 97980.953 & 7.1 & $1.679 \times 10^{-5}$ & $0.79(0.01)$ & $22(3)$ \\
\hline $\mathrm{CS}$ & $3-2$ & 146969.026 & 14.1 & $6.071 \times 10^{-5}$ & $2.11(0.02)$ & $17(2)$ \\
\hline $\mathrm{CS}$ & $5-4$ & 244935.555 & 35.3 & $2.981 \times 10^{-4}$ & $5.77(0.04)$ & $16.1(1.2)$ \\
\hline $\mathrm{CS}$ & $6-5$ & 293912.089 & 49.4 & $5.229 \times 10^{-4}$ & $5.87(0.04)$ & $17.4(1.0)$ \\
\hline $\mathrm{CS}$ & $7-6$ & 342882.854 & 65.8 & $8.395 \times 10^{-4}$ & $5.30(0.07)$ & $19.6(1.0)$ \\
\hline${ }^{13} \mathrm{CS}$ & $3-2$ & 138739.264 & 13.3 & $5.107 \times 10^{-5}$ & $0.17(0.01)$ & $13(3)$ \\
\hline${ }^{13} \mathrm{CS}$ & $5-4$ & 231220.684 & 33.3 & $2.507 \times 10^{-4}$ & $0.63(0.05)$ & $23(3)$ \\
\hline${ }^{13} \mathrm{CS}$ & $6-5$ & 277455.398 & 46.6 & $4.399 \times 10^{-4}$ & $0.70(0.06)$ & $15.4(1.1)$ \\
\hline $\mathrm{C}^{34} \mathrm{~S}$ & $3-2$ & 144617.101 & 13.9 & $5.784 \times 10^{-5}$ & $0.18(0.02)$ & $18(4)$ \\
\hline $\mathrm{C}^{34} \mathrm{~S}$ & $5-4$ & 241016.089 & 34.7 & $2.840 \times 10^{-4}$ & $0.41(0.04)$ & $22(4)$ \\
\hline $\mathrm{C}^{34} \mathrm{~S}$ & $6-5$ & 289209.067 & 48.6 & $4.982 \times 10^{-4}$ & $0.43(0.03)$ & $19(2)$ \\
\hline $\mathrm{o}-\mathrm{H}_{2} \mathrm{~S}$ & $1_{1,0}-1_{0,1}$ & 168762.754 & 8.1 & $2.677 \times 10^{-5}$ & $6.35(0.02)$ & $18(2)$ \\
\hline $\mathrm{o}-\mathrm{H}_{2} \mathrm{~S}$ & $4_{1,4}-3_{2,1}$ & 204140.204 & 144.5 & $3.006 \times 10^{-8}$ & $0.14(0.03)$ & $\mathrm{BF}$ \\
\hline $\mathrm{o}-\mathrm{H}_{2} \mathrm{~S}$ & $3_{3,0}-3_{2,1}$ & 300505.524 & 149.1 & $1.024 \times 10^{-4}$ & $1.05(0.07)$ & $17.3(1.1)$ \\
\hline $\mathrm{p}-\mathrm{H}_{2} \mathrm{~S}$ & $2,0-2_{1,1}$ & 216710.444 & 84.0 & $4.870 \times 10^{-5}$ & $0.66(0.05)$ & $18.3(1.4)$ \\
\hline $\mathrm{O}-{ }^{34} \mathrm{H}_{2} \mathrm{~S}$ & $1_{1,0}-1_{0,1}$ & 167910.516 & 8.1 & $2.616 \times 10^{-5}$ & $0.89(0.02)$ & $22(2)$ \\
\hline $\mathrm{SO}$ & $(2,2)-(1,1)$ & 86093.959 & 19.3 & $5.250 \times 10^{-6}$ & $0.70(0.03)$ & $17(3)$ \\
\hline SO & $(2,3)-(1,2)$ & 99299.891 & 9.2 & $1.125 \times 10^{-5}$ & $7.50(0.02)$ & $16(3)$ \\
\hline SO & $(3,2)-(2,1)$ & 109252.184 & 21.1 & $1.080 \times 10^{-5}$ & $1.11(0.02)$ & $17(3)$ \\
\hline SO & $(3,3)-(2,2)$ & 129138.904 & 25.5 & $2.250 \times 10^{-5}$ & $2.19(0.03)$ & $17(2)$ \\
\hline SO & $(3,4)-(2,3)$ & 138178.659 & 15.9 & $3.166 \times 10^{-5}$ & $12.43(0.02)$ & $17(2)$ \\
\hline SO & $(4,3)-(3,2)$ & 158971.816 & 28.7 & $4.233 \times 10^{-5}$ & $2.79(0.02)$ & $17(2)$ \\
\hline $\mathrm{SO}$ & $(4,4)-(3,3)$ & 172181.407 & 33.8 & $5.833 \times 10^{-5}$ & $2.77(0.05)$ & $17(2)$ \\
\hline SO & $(5,4)-(4,3)$ & 206176.019 & 38.6 & $1.010 \times 10^{-4}$ & $3.23(0.04)$ & $17.4(1.5)$ \\
\hline $\mathrm{SO}$ & $(5,5)-(4,4)$ & 215220.653 & 44.1 & $1.193 \times 10^{-4}$ & $3.76(0.04)$ & $16.9(1.4)$ \\
\hline SO & $(5,6)-(4,5)$ & 219949.396 & 35.0 & $1.335 \times 10^{-4}$ & $10.68(0.03)$ & $17.7(1.4)$ \\
\hline SO & $(6,5)-(5,4)$ & 251825.767 & 50.7 & $1.925 \times 10^{-4}$ & $3.15(0.04)$ & $16.1(1.2)$ \\
\hline $\mathrm{SO}$ & $(6,6)-(5,5)$ & 258255.830 & 56.5 & $2.120 \times 10^{-4}$ & $3.44(0.05)$ & $17.4(1.2)$ \\
\hline SO & $(6,7)-(5,6)$ & 261843.713 & 47.6 & $2.282 \times 10^{-4}$ & $7.54(0.07)$ & $16.2(1.1)$ \\
\hline SO & $(1,1)-(0,1)$ & 286340.151 & 15.2 & $1.403 \times 10^{-5}$ & $0.54(0.05)$ & $19(2)$ \\
\hline $\mathrm{SO}$ & $(7,6)-(6,5)$ & 296550.061 & 64.9 & $3.230 \times 10^{-4}$ & $2.31(0.03)$ & $16.7(1.2)$ \\
\hline SO & $(7,7)-(6,6)$ & 301286.123 & 71.0 & $3.429 \times 10^{-4}$ & $3.38(0.04)$ & $19.2(1.0)$ \\
\hline SO & $(7,8)-(6,7)$ & 304077.867 & 62.1 & $3.609 \times 10^{-4}$ & $7.23(0.05)$ & $16.2(1.0)$ \\
\hline SO & $(2,2)-(1,2)$ & 309502.443 & 19.3 & $1.419 \times 10^{-5}$ & $0.46(0.03)$ & $17.6(1.0)$ \\
\hline SO & $(3,3)-(2,3)$ & 339341.457 & 25.5 & $1.455 \times 10^{-5}$ & $0.45(0.06)$ & $16(2)$ \\
\hline SO & $(8,7)-(7,6)$ & 340714.295 & 81.2 & $4.985 \times 10^{-4}$ & $2.46(0.06)$ & $18.1(1.0)$ \\
\hline SO & $(8,8)-(7,7)$ & 344310.717 & 87.5 & $5.186 \times 10^{-4}$ & $2.72(0.08)$ & $18.2(1.0)$ \\
\hline SO & $(8,9)-(7,8)$ & 346528.537 & 78.8 & $5.382 \times 10^{-4}$ & $\mathrm{HB}$ & $\mathrm{HB}$ \\
\hline${ }^{34} \mathrm{SO}$ & $(2,3)-(1,2)$ & 97715.405 & 9.1 & $1.073 \times 10^{-5}$ & $0.51(0.02)$ & $18(3)$ \\
\hline${ }^{34} \mathrm{SO}$ & $(3,2)-(2,1)$ & 106743.368 & 20.9 & $1.007 \times 10^{-5}$ & $0.07(0.01)$ & $6(9)$ \\
\hline${ }^{34} \mathrm{SO}$ & $(3,4)-(2,3)$ & 135775.651 & 15.6 & $3.004 \times 10^{-5}$ & $0.93(0.02)$ & $18(2)$ \\
\hline${ }^{34} \mathrm{SO}$ & $(4,3)-(3,2)$ & 155506.808 & 28.4 & $3.961 \times 10^{-5}$ & $0.16(0.02)$ & $17(4)$ \\
\hline${ }^{34} \mathrm{SO}$ & $(4,4)-(3,3)$ & 168815.114 & 33.4 & $5.498 \times 10^{-5}$ & $0.17(0.02)$ & $20(4)$ \\
\hline${ }^{34} \mathrm{SO}$ & $(5,5)-(4,4)$ & 211013.024 & 43.5 & $1.124 \times 10^{-4}$ & $0.34(0.05)$ & $21(5)$ \\
\hline${ }^{34} \mathrm{SO}$ & $(5,6)-(4,5)$ & 215839.917 & 34.4 & $1.262 \times 10^{-4}$ & $0.74(0.04)$ & $17.6(1.4)$ \\
\hline${ }^{34} \mathrm{SO}$ & $(6,5)-(5,4)$ & 246663.403 & 49.9 & $1.809 \times 10^{-4}$ & $0.26(0.03)$ & $19(2)$ \\
\hline${ }^{34} \mathrm{SO}$ & $(6,6)-(5,5)$ & 253207.022 & 55.7 & $1.998 \times 10^{-4}$ & $0.28(0.04)$ & $21(3)$ \\
\hline${ }^{34} \mathrm{SO}$ & $(7,6)-(6,5)$ & 290562.257 & 63.8 & $3.037 \times 10^{-4}$ & $0.27(0.03)$ & $22(4)$ \\
\hline${ }^{34} \mathrm{SO}$ & $(7,8)-(6,7)$ & 298257.973 & 61.0 & $3.406 \times 10^{-4}$ & $0.50(0.03)$ & $15.6(1.4)$ \\
\hline
\end{tabular}


Table A.1. continued.

\begin{tabular}{|c|c|c|c|c|c|c|}
\hline Molecule & $\begin{array}{l}\text { Transition } \\
\text { QNs }\end{array}$ & $\begin{array}{l}v_{\text {rest }} \\
(\mathrm{MHz})\end{array}$ & $\begin{array}{l}E_{\mathrm{u}} \\
(\mathrm{K})\end{array}$ & $\begin{array}{l}A_{\mathrm{ul}} \\
\left(\mathrm{s}^{-1}\right)\end{array}$ & $\begin{array}{l}\int T_{\text {ant }}^{*} \mathrm{~d} v \\
\left(\mathrm{~K} \mathrm{~km} \mathrm{~s}^{-1}\right)\end{array}$ & $\begin{array}{l}v_{\exp } \\
\left(\mathrm{km} \mathrm{s}^{-1}\right)\end{array}$ \\
\hline $\mathrm{SO}_{2}$ & $8_{1,7}-8_{0,8}$ & 83688.092 & 36.7 & $6.825 \times 10^{-6}$ & $1.32(0.03)$ & $20(4)$ \\
\hline $\mathrm{SO}_{2}$ & $22,0-3,3$ & 100878.107 & 12.6 & $1.026 \times 10^{-6}$ & $0.11(0.01)$ & $20(6)$ \\
\hline $\mathrm{SO}_{2}$ & $3_{1,3}-2_{0,2}$ & 104029.420 & 7.7 & $1.006 \times 10^{-5}$ & $1.73(0.03)$ & $21(3)$ \\
\hline $\mathrm{SO}_{2}$ & $10_{1,9}-10_{0,10}$ & 104239.299 & 54.7 & $1.122 \times 10^{-5}$ & $1.12(0.02)$ & $19(3)$ \\
\hline $\mathrm{SO}_{2}$ & $12_{2,10}-12_{1,11}$ & 128605.111 & 82.6 & $2.615 \times 10^{-5}$ & $0.64(0.02)$ & $16(2)$ \\
\hline $\mathrm{SO}_{2}$ & $12_{1,11}-11_{2,10}$ & 129105.786 & 76.4 & $9.029 \times 10^{-6}$ & $0.64(0.02)$ & $19(2)$ \\
\hline $\mathrm{SO}_{2}$ & $10_{2,8}-10_{1,9}$ & 129514.799 & 60.9 & $2.502 \times 10^{-5}$ & $0.92(0.03)$ & $16(2)$ \\
\hline $\mathrm{SO}_{2}$ & $12_{1,11}-12_{0,12}$ & 131014.841 & 76.4 & $1.856 \times 10^{-5}$ & $1.00(0.01)$ & $22(2)$ \\
\hline $\mathrm{SO}_{2}$ & $14_{2,12}-14_{1,13}$ & 132744.832 & 108.1 & $2.933 \times 10^{-5}$ & $0.47(0.03)$ & $18(2)$ \\
\hline $\mathrm{SO}_{2}$ & $8_{2,6}-8_{1,7}$ & 134004.812 & 43.1 & $2.501 \times 10^{-5}$ & $1.28(0.02)$ & $16(2)$ \\
\hline $\mathrm{SO}_{2}$ & $5_{1,5}-4_{0,4}$ & 135696.017 & 15.7 & $2.208 \times 10^{-5}$ & $3.78(0.03)$ & $17(2)$ \\
\hline $\mathrm{SO}_{2}$ & $6_{2,4}-6_{1,5}$ & 140306.166 & 29.2 & $2.528 \times 10^{-5}$ & $1.76(0.02)$ & $17(2)$ \\
\hline $\mathrm{SO}_{2}$ & $16_{2,14}-16_{1,15}$ & 143057.080 & 137.5 & $3.572 \times 10^{-5}$ & $0.31(0.02)$ & $18(3)$ \\
\hline $\mathrm{SO}_{2}$ & $4_{2,2}-4_{1,3}$ & 146605.520 & 19.0 & $2.470 \times 10^{-5}$ & $1.81(0.03)$ & $17(2)$ \\
\hline $\mathrm{SO}_{2}$ & $2_{2,0}-2_{1,1}$ & 151378.663 & 12.6 & $1.875 \times 10^{-5}$ & $1.11(0.03)$ & $18(2)$ \\
\hline $\mathrm{SO}_{2}$ & $3_{2,2}-3_{1,3}$ & 158199.781 & 15.3 & $2.532 \times 10^{-5}$ & $1.58(0.02)$ & $17(2)$ \\
\hline $\mathrm{SO}_{2}$ & $18_{2,16}-18_{1,17}$ & 160342.971 & 170.8 & $4.692 \times 10^{-5}$ & $0.33(0.02)$ & $13(2)$ \\
\hline $\mathrm{SO}_{2}$ & $4_{3,1}-5_{2,4}$ & 160543.024 & 31.3 & $4.321 \times 10^{-6}$ & $0.06(0.01)$ & $8(4)$ \\
\hline $\mathrm{SO}_{2}$ & $10_{0,10}-9_{1,9}$ & 160827.841 & 49.7 & $3.954 \times 10^{-5}$ & $3.37(0.02)$ & $17(2)$ \\
\hline $\mathrm{SO}_{2}$ & $14_{1,13}-14_{0,14}$ & 163605.533 & 101.8 & $3.006 \times 10^{-5}$ & $1.33(0.03)$ & $18(2)$ \\
\hline $\mathrm{SO}_{2}$ & $5_{2,4}-5_{1,5}$ & 165144.652 & 23.6 & $3.122 \times 10^{-5}$ & $2.47(0.02)$ & $18(2)$ \\
\hline $\mathrm{SO}_{2}$ & $7,7-6_{0,6}$ & 165225.452 & 27.1 & $4.135 \times 10^{-5}$ & $6.14(0.02)$ & $17(2)$ \\
\hline $\mathrm{SO}_{2}$ & $12_{0,12}-11_{1,11}$ & 203391.484 & 70.1 & $8.805 \times 10^{-5}$ & $4.04(0.06)$ & $16.4(1.5)$ \\
\hline $\mathrm{SO}_{2}$ & $18_{3,15}-18_{2,16}$ & 204246.762 & 180.6 & $9.267 \times 10^{-5}$ & $0.38(0.05)$ & $15(3)$ \\
\hline $\mathrm{SO}_{2}$ & $11_{2,10}-11_{1,11}$ & 205300.539 & 70.2 & $5.318 \times 10^{-5}$ & $1.54(0.06)$ & $17.1(1.5)$ \\
\hline $\mathrm{SO}_{2}$ & $3_{2,2}-2_{1,1}$ & 208700.337 & 15.3 & $6.719 \times 10^{-5}$ & $3.08(0.03)$ & $17.2(1.4)$ \\
\hline $\mathrm{SO}_{2}$ & $26_{3,23}-26_{2,24}$ & 213068.427 & 350.8 & $1.156 \times 10^{-4}$ & $0.19(0.04)$ & $5(3)$ \\
\hline $\mathrm{SO}_{2}$ & $16_{3,13}-16_{2,14}$ & 214689.395 & 147.8 & $9.902 \times 10^{-5}$ & $0.32(0.03)$ & $19(2)$ \\
\hline $\mathrm{SO}_{2}$ & $22_{2,20}-22_{1,21}$ & 216643.304 & 248.5 & $9.271 \times 10^{-5}$ & $0.40(0.04)$ & $11(2)$ \\
\hline $\mathrm{SO}_{2}$ & $11_{1,11}-10_{0,10}$ & 221965.221 & 60.4 & $1.138 \times 10^{-4}$ & $5.81(0.06)$ & $16.2(1.4)$ \\
\hline $\mathrm{SO}_{2}$ & $13_{2,12}-13_{1,13}$ & 225153.705 & 93.0 & $6.523 \times 10^{-5}$ & $0.94(0.07)$ & $17.3(1.4)$ \\
\hline $\mathrm{SO}_{2}$ & $14_{3,11}-14_{2,12}$ & 226300.028 & 119.0 & $1.068 \times 10^{-4}$ & $\mathrm{HB}$ & $\mathrm{HB}$ \\
\hline $\mathrm{SO}_{2}$ & $4_{2,2}-3_{1,3}$ & 235151.721 & 19.0 & $7.691 \times 10^{-5}$ & $4.41(0.05)$ & $17.2(1.3)$ \\
\hline $\mathrm{SO}_{2}$ & $16_{1,15}-15_{2,14}$ & 236216.688 & 130.7 & $7.505 \times 10^{-5}$ & $0.66(0.05)$ & $17.0(1.4)$ \\
\hline $\mathrm{SO}_{2}$ & $12_{3,9}-12_{2,10}$ & 237068.834 & 94.0 & $1.141 \times 10^{-4}$ & $0.85(0.04)$ & $18.6(1.4)$ \\
\hline $\mathrm{SO}_{2}$ & $18_{1,17}-18_{0,18}$ & 240942.792 & 163.1 & $7.024 \times 10^{-5}$ & $0.47(0.04)$ & $18(3)$ \\
\hline $\mathrm{SO}_{2}$ & $5_{2,4}-4_{1,3}$ & 241615.798 & 23.6 & $8.455 \times 10^{-5}$ & $4.74(0.06)$ & $17.6(1.2)$ \\
\hline $\mathrm{SO}_{2}$ & $14_{0,14}-13_{1,13}$ & 244254.220 & 93.9 & $1.639 \times 10^{-4}$ & $3.71(0.04)$ & $17.3(1.2)$ \\
\hline $\mathrm{SO}_{2}$ & $10_{3,7}-10_{2,8}$ & 245563.423 & 72.7 & $1.191 \times 10^{-4}$ & $1.31(0.04)$ & $17.0(1.2)$ \\
\hline $\mathrm{SO}_{2}$ & $15_{2,14}-15_{1,15}$ & 248057.403 & 119.3 & $8.056 \times 10^{-5}$ & $0.66(0.05)$ & $16.0(1.3)$ \\
\hline $\mathrm{SO}_{2}$ & $13_{1,13}-12_{0,12}$ & 251199.676 & 82.2 & $1.756 \times 10^{-4}$ & $\mathrm{HB}$ & $\mathrm{HB}$ \\
\hline $\mathrm{SO}_{2}$ & $8_{3,5}-8_{2,6}$ & 251210.586 & 55.2 & $1.198 \times 10^{-4}$ & $\mathrm{HB}$ & $\mathrm{HB}$ \\
\hline $\mathrm{SO}_{2}$ & $6_{3,3}-6_{2,4}$ & 254280.537 & 41.4 & $1.136 \times 10^{-4}$ & $2.02(0.03)$ & $16.7(1.2)$ \\
\hline $\mathrm{SO}_{2}$ & $4_{3,1}-4_{2,2}$ & 255553.303 & 31.3 & $9.284 \times 10^{-5}$ & $1.62(0.06)$ & $17.2(1.2)$ \\
\hline $\mathrm{SO}_{2}$ & $3_{3,1}-3_{2,2}$ & 255958.045 & 27.6 & $6.626 \times 10^{-5}$ & $1.09(0.06)$ & $17.6(1.2)$ \\
\hline $\mathrm{SO}_{2}$ & $5_{3,3}-5_{2,4}$ & 256246.946 & 35.9 & $1.074 \times 10^{-4}$ & $1.94(0.04)$ & $17.2(1.2)$ \\
\hline $\mathrm{SO}_{2}$ & $7_{3,5}-7_{2,6}$ & 257099.967 & 47.8 & $1.223 \times 10^{-4}$ & $1.78(0.04)$ & $16.5(1.2)$ \\
\hline $\mathrm{SO}_{2}$ & $32_{4,28}-32_{3,29}$ & 258388.714 & 531.1 & $2.102 \times 10^{-4}$ & $0.19(0.03)$ & $6.2(1.4)$ \\
\hline $\mathrm{SO}_{2}$ & $9_{3,7}-9_{2,8}$ & 258942.200 & 63.5 & $1.318 \times 10^{-4}$ & $1.53(0.04)$ & $17.2(1.2)$ \\
\hline $\mathrm{SO}_{2}$ & $30_{4,26}-30_{3,27}$ & 259599.446 & 471.5 & $2.072 \times 10^{-4}$ & $0.16(0.02)$ & $6(2)$ \\
\hline $\mathrm{SO}_{2}$ & $11_{3,9}-11_{2,10}$ & 262256.907 & 82.8 & $1.408 \times 10^{-4}$ & $0.88(0.09)$ & $18(2)$ \\
\hline $\mathrm{SO}_{2}$ & $30_{3,27}-30_{2,28}$ & 263543.954 & 459.1 & $1.894 \times 10^{-4}$ & $0.21(0.02)$ & $5.1(1.4)$ \\
\hline $\mathrm{SO}_{2}$ & $34_{4,30}-34_{3,31}$ & 265481.970 & 594.7 & $2.277 \times 10^{-4}$ & $0.12(0.02)$ & $8.6(1.2)$ \\
\hline
\end{tabular}


Table A.1. continued.

\begin{tabular}{|c|c|c|c|c|c|c|}
\hline Molecule & $\begin{array}{l}\text { Transition } \\
\text { QNs }\end{array}$ & $\begin{array}{l}v_{\text {rest }} \\
(\mathrm{MHz})\end{array}$ & $\begin{array}{l}E_{\mathrm{u}} \\
(\mathrm{K})\end{array}$ & $\begin{array}{l}A_{\mathrm{ul}} \\
\left(\mathrm{s}^{-1}\right)\end{array}$ & $\begin{array}{l}\int T_{\text {ant }}^{*} \mathrm{~d} v \\
\left(\mathrm{~K} \mathrm{~km} \mathrm{~s}^{-1}\right)\end{array}$ & $\begin{array}{l}v_{\exp } \\
\left(\mathrm{km} \mathrm{s}^{-1}\right)\end{array}$ \\
\hline $\mathrm{SO}_{2}$ & $13_{3,11}-13_{2,12}$ & 267537.453 & 105.8 & $1.513 \times 10^{-4}$ & $0.88(0.05)$ & $15.8(1.2)$ \\
\hline $\mathrm{SO}_{2}$ & $28_{4,24}-28_{3,25}$ & 267719.839 & 415.9 & $2.157 \times 10^{-4}$ & $0.11(0.04)$ & $12(2)$ \\
\hline $\mathrm{SO}_{2}$ & $7_{2,6}-6_{1,5}$ & 271529.016 & 35.5 & $1.107 \times 10^{-4}$ & $2.53(0.07)$ & $16.8(1.1)$ \\
\hline $\mathrm{SO}_{2}$ & $17_{2,16}-17_{1,17}$ & 273752.962 & 149.2 & $9.966 \times 10^{-5}$ & $0.29(0.06)$ & $12(2)$ \\
\hline $\mathrm{SO}_{2}$ & $15_{3,13}-15_{2,14}$ & 275240.185 & 132.5 & $1.645 \times 10^{-4}$ & $0.64(0.08)$ & $8(4)$ \\
\hline $\mathrm{SO}_{2}$ & $26_{4,22}-26_{3,23}$ & 280807.246 & 364.3 & $2.329 \times 10^{-4}$ & $0.22(0.05)$ & $4.8(1.3)$ \\
\hline $\mathrm{SO}_{2}$ & $36_{4,32}-36_{3,33}$ & 281688.930 & 662.1 & $2.636 \times 10^{-4}$ & $0.22(0.03)$ & $9(2)$ \\
\hline $\mathrm{SO}_{2}$ & $15_{1,15}-14_{0,14}$ & 281762.602 & 107.4 & $2.609 \times 10^{-4}$ & $4.64(0.05)$ & $17.1(1.1)$ \\
\hline $\mathrm{SO}_{2}$ & $6_{2,4}-5_{1,5}$ & 282036.568 & 29.2 & $1.003 \times 10^{-4}$ & $4.33(0.05)$ & $17.7(1.1)$ \\
\hline $\mathrm{SO}_{2}$ & $20_{1,19}-20_{0,20}$ & 282292.806 & 198.9 & $1.002 \times 10^{-4}$ & $0.40(0.05)$ & $16(2)$ \\
\hline $\mathrm{SO}_{2}$ & $16_{0,16}-15_{1,15}$ & 283464.770 & 121.0 & $2.700 \times 10^{-4}$ & $3.80(0.03)$ & $22.0(1.1)$ \\
\hline $\mathrm{SO}_{2}$ & $17_{3,15}-17_{2,16}$ & 285743.589 & 162.9 & $1.816 \times 10^{-4}$ & $0.78(0.06)$ & $17.5(1.0)$ \\
\hline $\mathrm{SO}_{2}$ & $22_{2,20}-21_{3,19}$ & 286416.272 & 248.5 & $9.293 \times 10^{-5}$ & $0.30(0.06)$ & $16(2)$ \\
\hline $\mathrm{SO}_{2}$ & $18_{1,17}-17_{2,16}$ & 288519.997 & 163.1 & $1.570 \times 10^{-4}$ & $1.00(0.06)$ & $16.3(1.1)$ \\
\hline $\mathrm{SO}_{2}$ & $26_{2,24}-26_{1,25}$ & 296168.675 & 340.6 & $1.873 \times 10^{-4}$ & $0.21(0.02)$ & $10.2(1.4)$ \\
\hline $\mathrm{SO}_{2}$ & $9_{2,8}-8_{1,7}$ & 298576.309 & 51.0 & $1.442 \times 10^{-4}$ & $3.35(0.04)$ & $17.1(1.0)$ \\
\hline $\mathrm{SO}_{2}$ & $19_{3,17}-19_{2,18}$ & 299316.820 & 197.0 & $2.037 \times 10^{-4}$ & $0.55(0.06)$ & $17(2)$ \\
\hline $\mathrm{SO}_{2}$ & $32_{3,29}-32_{2,30}$ & 300273.420 & 518.7 & $2.547 \times 10^{-4}$ & $0.13(0.03)$ & $6(2)$ \\
\hline $\mathrm{SO}_{2}$ & $19_{2,18}-19_{1,19}$ & 301896.629 & 182.6 & $1.229 \times 10^{-4}$ & $0.35(0.06)$ & $20(3)$ \\
\hline $\mathrm{SO}_{2}$ & $38_{4,34}-38_{3,35}$ & 307185.315 & 733.4 & $3.228 \times 10^{-4}$ & $0.41(0.06)$ & $9(2)$ \\
\hline $\mathrm{SO}_{2}$ & $22_{4,18}-22_{3,19}$ & 312542.520 & 272.8 & $2.819 \times 10^{-4}$ & $0.27(0.03)$ & $9(2)$ \\
\hline $\mathrm{SO}_{2}$ & $3_{3,1}-2_{2,0}$ & 313279.719 & 27.6 & $3.396 \times 10^{-4}$ & $3.77(0.07)$ & $18.2(1.0)$ \\
\hline $\mathrm{SO}_{2}$ & $17_{1,17}-16_{0,16}$ & 313660.853 & 136.1 & $3.747 \times 10^{-4}$ & $1.87(0.05)$ & $16.5(1.0)$ \\
\hline $\mathrm{SO}_{2}$ & $21_{3,19}-21_{2,20}$ & 316098.875 & 234.7 & $2.319 \times 10^{-4}$ & $0.35(0.04)$ & $5(2)$ \\
\hline $\mathrm{SO}_{2}$ & $18_{0,18}-17_{1,17}$ & 321330.168 & 151.5 & $4.087 \times 10^{-4}$ & $1.5(0.2)$ & $11(2)$ \\
\hline $\mathrm{SO}_{2}$ & $4_{3,1}-3_{2,2}$ & 332505.243 & 31.3 & $3.290 \times 10^{-4}$ & $3.01(0.09)$ & $17.0(1.0)$ \\
\hline $\mathrm{SO}_{2}$ & $8,6-7,7$ & 334673.355 & 43.1 & $1.270 \times 10^{-4}$ & $2.5(0.2)$ & $21(2)$ \\
\hline $\mathrm{SO}_{2}$ & $18_{4,14}-18_{3,15}$ & 338305.994 & 196.8 & $3.266 \times 10^{-4}$ & $0.44(0.06)$ & $17(2)$ \\
\hline $\mathrm{SO}_{2}$ & $20_{1,19}-19_{2,18}$ & 338611.811 & 198.9 & $2.868 \times 10^{-4}$ & $0.48(0.06)$ & $9(2)$ \\
\hline $\mathrm{SO}_{2}$ & $28_{2,26}-28_{1,27}$ & 340316.406 & 391.8 & $2.580 \times 10^{-4}$ & $0.36(0.04)$ & $10.8(1.0)$ \\
\hline $\mathrm{SO}_{2}$ & $36_{5,31}-36_{4,32}$ & 341673.955 & 678.5 & $4.344 \times 10^{-4}$ & $0.18(0.03)$ & $4(2)$ \\
\hline $\mathrm{SO}_{2}$ & $34_{3,31}-34_{2,32}$ & 342761.629 & 581.9 & $3.447 \times 10^{-4}$ & $0.18(0.03)$ & $4(2)$ \\
\hline $\mathrm{SO}_{2}$ & $13_{2,12}-12_{1,11}$ & 345338.540 & 93.0 & $2.381 \times 10^{-4}$ & HB & $\mathrm{HB}$ \\
\hline $\mathrm{SO}_{2}$ & $16_{4,12}-16_{3,13}$ & 346523.879 & 164.5 & $3.390 \times 10^{-4}$ & $\mathrm{HB}$ & $\mathrm{HB}$ \\
\hline $\mathrm{SO}_{2}$ & $19_{1,19}-18_{0,18}$ & 346652.171 & 168.1 & $5.219 \times 10^{-4}$ & $1.43(0.12)$ & $19(3)$ \\
\hline $\mathrm{SO}_{2}$ & $5_{3,3}-4_{2,2}$ & 351257.225 & 35.9 & $3.357 \times 10^{-4}$ & $2.05(0.12)$ & $17.2(1.0)$ \\
\hline${ }^{34} \mathrm{SO}_{2}$ & $3_{1,3}-2_{0,2}$ & 102031.880 & 7.6 & $9.495 \times 10^{-6}$ & $0.17(0.02)$ & $24(6)$ \\
\hline${ }^{34} \mathrm{SO}_{2}$ & $8_{2,6}-8_{1,7}$ & 128668.791 & 42.8 & $2.245 \times 10^{-5}$ & $0.054(0.015)$ & $10(6)$ \\
\hline${ }^{34} \mathrm{SO}_{2}$ & $5_{1,5}-4_{0,4}$ & 133471.429 & 15.5 & $2.107 \times 10^{-5}$ & $0.19(0.02)$ & $16(6)$ \\
\hline${ }^{34} \mathrm{SO}_{2}$ & $4_{3,1}-5_{2,4}$ & 151917.559 & 30.5 & $3.663 \times 10^{-6}$ & $0.07(0.02)$ & $13(5)$ \\
\hline${ }^{34} \mathrm{SO}_{2}$ & $3_{2,2}-3_{1,3}$ & 153015.053 & 15.0 & $2.290 \times 10^{-5}$ & $0.11(0.02)$ & $12(5)$ \\
\hline${ }^{34} \mathrm{SO}_{2}$ & $18_{2,16}-18_{1,17}$ & 160802.573 & 170.3 & $4.611 \times 10^{-5}$ & $0.04(0.01)$ & $4(4)$ \\
\hline${ }^{34} \mathrm{SO}_{2}$ & $10_{0,10}-9_{1,9}$ & 162020.378 & 49.5 & $4.100 \times 10^{-5}$ & $0.24(0.02)$ & $17(2)$ \\
\hline${ }^{34} \mathrm{SO}_{2}$ & $7_{1,7}-6_{0,6}$ & 162775.882 & 26.9 & $3.976 \times 10^{-5}$ & $0.35(0.02)$ & $17(2)$ \\
\hline${ }^{34} \mathrm{SO}_{2}$ & $11_{1,11}-10_{0,10}$ & 219355.012 & 60.1 & $1.110 \times 10^{-4}$ & $0.32(0.02)$ & $15.2(1.4)$ \\
\hline${ }^{34} \mathrm{SO}_{2}$ & $13_{1,13}-12_{0,12}$ & 248698.698 & 81.8 & $1.721 \times 10^{-4}$ & $0.26(0.04)$ & $18(2)$ \\
\hline${ }^{34} \mathrm{SO}_{2}$ & $7_{2,6}-6_{1,5}$ & 265554.053 & 35.1 & $1.038 \times 10^{-4}$ & $0.52(0.04)$ & $27(2)$ \\
\hline $\mathrm{PN}$ & $2-1$ & 93979.768 & 6.8 & $2.916 \times 10^{-5}$ & $0.18(0.02)$ & $16(3)$ \\
\hline $\mathrm{PN}$ & $3-2$ & 140967.690 & 13.5 & $1.054 \times 10^{-4}$ & $0.48(0.02)$ & $17(2)$ \\
\hline $\mathrm{PN}$ & $5-4$ & 234935.691 & 33.8 & $5.177 \times 10^{-4}$ & $0.82(0.05)$ & $14.2(1.4)$ \\
\hline PN & $6-5$ & 281914.200 & 47.4 & $9.083 \times 10^{-4}$ & $0.99(0.06)$ & $18.6(1.1)$ \\
\hline
\end{tabular}


Table A.1. continued.

\begin{tabular}{|c|c|c|c|c|c|c|}
\hline Molecule & $\begin{array}{l}\text { Transition } \\
\text { QNs }\end{array}$ & $\begin{array}{l}v_{\text {rest }} \\
(\mathrm{MHz})\end{array}$ & $\begin{array}{l}E_{\mathrm{u}} \\
(\mathrm{K})\end{array}$ & $\begin{array}{l}A_{\mathrm{ul}} \\
\left(\mathrm{s}^{-1}\right)\end{array}$ & $\begin{array}{l}\int T_{\text {ant }}^{*} \mathrm{~d} v \\
\left(\mathrm{~K} \mathrm{~km} \mathrm{~s}^{-1}\right)\end{array}$ & $\begin{array}{l}v_{\exp } \\
\left(\mathrm{km} \mathrm{s}^{-1}\right)\end{array}$ \\
\hline $\mathrm{PO}$ & $\Omega=1 / 2, \mathrm{e},(5 / 2,3)-(3 / 2,2)$ & 108998.445 & 8.4 & $2.132 \times 10^{-5}$ & $0.08(0.02)$ & $6(3)$ \\
\hline $\mathrm{PO}$ & $\Omega=1 / 2, \mathrm{f},(5 / 2,3)-(3 / 2,2)$ & 109206.200 & 8.4 & $2.143 \times 10^{-5}$ & $0.07(0.02)$ & $7(3)$ \\
\hline $\mathrm{PO}$ & $\Omega=1 / 2, \mathrm{e},(7 / 2,4)-(5 / 2,3)$ & 152656.979 & 15.7 & $6.274 \times 10^{-5}$ & $0.28(0.03)$ & $\mathrm{BF}$ \\
\hline PO & $\Omega=1 / 2, \mathrm{e},(7 / 2,3)-(5 / 2,2)$ & 152680.282 & 15.7 & $5.975 \times 10^{-5}$ & $0.14(0.02)$ & $\mathrm{BF}$ \\
\hline $\mathrm{PO}$ & $\Omega=1 / 2, \mathrm{f},(7 / 2,4)-(5 / 2,3)$ & 152855.454 & 15.8 & $6.296 \times 10^{-5}$ & $0.14(0.02)$ & $13(4)$ \\
\hline $\mathrm{PO}$ & $\Omega=1 / 2, \mathrm{f},(7 / 2,3)-(5 / 2,2)$ & 152888.128 & 15.7 & $5.998 \times 10^{-5}$ & $0.16(0.02)$ & $15(4)$ \\
\hline PO & $\Omega=1 / 2, \mathrm{e},(11 / 2,5)-(9 / 2,5)$ & 239704.364 & 36.7 & $4.699 \times 10^{-6}$ & $0.06(0.02)$ & $5(3)$ \\
\hline $\mathrm{PO}$ & $\Omega=1 / 2, \mathrm{f},(11 / 2,6)-(9 / 2,5)$ & 239948.978 & 36.7 & $\left.2.583 \times 10^{-4}\right)$ & \multirow{2}{*}{$0.33(0.04)$} & \multirow{2}{*}{ HB } \\
\hline PO & $\Omega=1 / 2, \mathrm{f},(11 / 2,5)-(9 / 2,4)$ & 239958.096 & 36.7 & $\left.2.536 \times 10^{-4}\right\}$ & & \\
\hline $\mathrm{PO}$ & $\Omega=1 / 2, \mathrm{e},(11 / 2,6)-(9 / 2,5)$ & 240141.054 & 36.7 & $2.590 \times 10^{-4}$ & \multirow{2}{*}{$0.25(0.04)$} & \multirow{2}{*}{ HB } \\
\hline $\mathrm{PO}$ & $\Omega=1 / 2, \mathrm{e},(11 / 2,5)-(9 / 2,4)$ & 240152.530 & 36.7 & $\left.2.543 \times 10^{-4}\right\}$ & & \\
\hline $\mathrm{PO}$ & $\Omega=1 / 2, \mathrm{f},(13 / 2,7)-(11 / 2,6)$ & 283586.816 & 50.3 & $4.330 \times 10^{-4}$ & \multirow{2}{*}{$0.44(0.03)$} & \multirow{2}{*}{$\mathrm{HB}$} \\
\hline $\mathrm{PO}$ & $\Omega=1 / 2, \mathrm{f},(13 / 2,6)-(11 / 2,5)$ & 283593.166 & 50.3 & $4.274 \times 10^{-4}$ & & \\
\hline $\mathrm{PO}$ & $\Omega=1 / 2, \mathrm{e},(13 / 2,7)-(11 / 2,6)$ & 283777.587 & 50.3 & $4.339 \times 10^{-4}$ & \multirow{2}{*}{$0.44(0.03)$} & \multirow{2}{*}{$\mathrm{HB}$} \\
\hline $\mathrm{PO}$ & $\Omega=1 / 2, \mathrm{e},(13 / 2,6)-(11 / 2,5)$ & 283785.404 & 50.3 & $4.283 \times 10^{-4}$ & & \\
\hline NO & $\Omega=1 / 2, \mathrm{e},(5 / 2,7 / 2)-(3 / 2,5 / 2)$ & 250436.842 & 19.2 & $\left.1.841 \times 10^{-6}\right)$ & \multirow{3}{*}{$0.47(0.05)$} & \multirow[b]{4}{*}{$20(2)$} \\
\hline NO & $\Omega=1 / 2, \mathrm{e},(5 / 2,5 / 2)-(3 / 2,3 / 2)$ & 250440.653 & 19.2 & $1.547 \times 10^{-6}$ & & \\
\hline NO & $\Omega=1 / 2, \mathrm{e},(5 / 2,3 / 2)-(3 / 2,1 / 2)$ & 250448.526 & 19.2 & $1.381 \times 10^{-6}$ & & \\
\hline NO & $\Omega=1 / 2, \mathrm{e},(5 / 2,3 / 2)-(3 / 2,5 / 2)$ & 250517.704 & 19.2 & $1.841 \times 10^{-8}$ & $0.17(0.04)$ & \\
\hline $\mathrm{o}-\mathrm{H}_{2} \mathrm{CO}$ & $2_{1,2}-1_{1,1}$ & 140839.516 & 6.8 & $5.296 \times 10^{-5}$ & $0.41(0.02)$ & $20(2)$ \\
\hline $\mathrm{o}-\mathrm{H}_{2} \mathrm{CO}$ & $2,1-1_{1,0}$ & 150498.335 & 7.5 & $6.462 \times 10^{-5}$ & $0.43(0.03)$ & $13(2)$ \\
\hline $\mathrm{o}-\mathrm{H}_{2} \mathrm{CO}$ & $3_{1,3}-2_{1,2}$ & 211211.449 & 16.9 & $2.268 \times 10^{-4}$ & $0.71(0.05)$ & $16.6(1.5)$ \\
\hline $\mathrm{O}-\mathrm{H}_{2} \mathrm{CO}$ & $3_{1,2}-2_{1,1}$ & 225697.772 & 18.3 & $2.767 \times 10^{-4}$ & $0.61(0.06)$ & $18(3)$ \\
\hline $\mathrm{O}-\mathrm{H}_{2} \mathrm{CO}$ & $4_{1,4}-3_{1,3}$ & 281526.919 & 30.4 & $5.874 \times 10^{-4}$ & $0.75(0.06)$ & $25(2)$ \\
\hline $\mathrm{o}-\mathrm{H}_{2} \mathrm{CO}$ & $4_{3,2}-3_{3,1}$ & 291380.441 & 125.8 & $3.040 \times 10^{-4}$ & $0.19(0.02)$ & $\mathrm{BF}$ \\
\hline $\mathrm{o}-\mathrm{H}_{2} \mathrm{CO}$ & $4_{3,1}-3_{3,0}$ & 291384.360 & 125.8 & $3.040 \times 10^{-4}$ & $0.29(0.02)$ & $\mathrm{BF}$ \\
\hline $\mathrm{o}-\mathrm{H}_{2} \mathrm{CO}$ & $4_{1,3}-3_{1,2}$ & 300836.630 & 32.7 & $7.168 \times 10^{-4}$ & $0.63(0.11)$ & $15.1(1.0)$ \\
\hline $\mathrm{p}-\mathrm{H}_{2} \mathrm{CO}$ & $2_{0,2}-1_{0,1}$ & 145602.951 & 10.5 & $7.802 \times 10^{-5}$ & $0.28(0.02)$ & $25(3)$ \\
\hline $\mathrm{p}-\mathrm{H}_{2} \mathrm{CO}$ & $3_{0,3}-2_{0,2}$ & 218222.187 & 21.0 & $2.814 \times 10^{-4}$ & $0.26(0.03)$ & $24(3)$ \\
\hline $\mathrm{p}-\mathrm{H}_{2} \mathrm{CO}$ & $3_{2,2}-2_{2,1}$ & 218475.634 & 68.1 & $1.569 \times 10^{-4}$ & $0.41(0.04)$ & $17(2)$ \\
\hline $\mathrm{p}-\mathrm{H}_{2} \mathrm{CO}$ & $4_{0,4}-3_{0,3}$ & 290623.412 & 34.9 & $6.891 \times 10^{-4}$ & $0.31(0.03)$ & $24(2)$ \\
\hline $\mathrm{p}-\mathrm{H}_{2} \mathrm{CO}$ & $4_{2,2}-3_{2,1}$ & 291948.066 & 82.1 & $5.241 \times 10^{-4}$ & $0.15(0.03)$ & $14(2)$ \\
\hline $\mathrm{CN}$ & $(1,1 / 2,1 / 2)-(0,1 / 2,3 / 2)$ & 113144.157 & 5.4 & $1.053 \times 10^{-6}$ & $0.19(0.03)$ & $11(3)$ \\
\hline $\mathrm{CN}$ & $(1,1 / 2,3 / 2)-(0,1 / 2,1 / 2)$ & 113170.492 & 5.4 & $5.145 \times 10^{-6}$ & $0.18(0.03)$ & $15(3)$ \\
\hline $\mathrm{CN}$ & $(1,1 / 2,3 / 2)-(0,1 / 2,3 / 2)$ & 113191.279 & 5.4 & $6.682 \times 10^{-6}$ & $0.20(0.03)$ & $18(4)$ \\
\hline $\mathrm{CN}$ & $(1,3 / 2,3 / 2)-(0,1 / 2,1 / 2)$ & 113488.120 & 5.4 & $6.736 \times 10^{-6}$ & \multirow{2}{*}{$0.47(0.03)$} & \multirow{2}{*}{$\mathrm{HB}$} \\
\hline $\mathrm{CN}$ & $(1,3 / 2,5 / 2)-(0,1 / 2,3 / 2)$ & 113490.970 & 5.4 & $\left.1.192 \times 10^{-5}\right\}$ & & \\
\hline $\mathrm{CN}$ & $(2,3 / 2,1 / 2)-(1,3 / 2,3 / 2)$ & 226298.943 & 16.3 & $8.227 \times 10^{-6}$ & \multirow{2}{*}{ HB } & \multirow{2}{*}{ HB } \\
\hline $\mathrm{CN}$ & $(2,3 / 2,3 / 2)-(1,3 / 2,1 / 2)$ & 226303.037 & 16.3 & $4.169 \times 10^{-6}$ & & \\
\hline $\mathrm{CN}$ & $(2,3 / 2,1 / 2)-(1,1 / 2,3 / 2)$ & 226616.571 & 16.3 & $1.073 \times 10^{-5}$ & $0.21(0.03)$ & $20.9(1.5)$ \\
\hline $\mathrm{CN}$ & $(2,3 / 2,3 / 2)-(1,1 / 2,3 / 2)$ & 226632.190 & 16.3 & $4.259 \times 10^{-5}$ & $0.28(0.02)$ & $11(3)$ \\
\hline $\mathrm{CN}$ & $(2,3 / 2,5 / 2)-(1,1 / 2,3 / 2)$ & 226659.558 & 16.3 & $\left.9.467 \times 10^{-5}\right)$ & \multirow{2}{*}{$1.32(0.04)$} & \multirow{2}{*}{ HB } \\
\hline $\mathrm{CN}$ & $(2,3 / 2,1 / 2)-(1,1 / 2,1 / 2)$ & 226663.693 & 16.3 & $\left.8.465 \times 10^{-5}\right\}$ & & \\
\hline $\mathrm{CN}$ & $(2,3 / 2,3 / 2)-(1,1 / 2,1 / 2)$ & 226679.311 & 16.3 & $5.268 \times 10^{-5}$ & $0.26(0.03)$ & $10.9(1.1)$ \\
\hline $\mathrm{CN}$ & $(2,5 / 2,5 / 2)-(1,3 / 2,3 / 2)$ & 226874.191 & 16.3 & $9.621 \times 10^{-5}$ & \multirow{4}{*}{$1.60(0.04)$} & \multirow{4}{*}{ HB } \\
\hline $\mathrm{CN}$ & $(2,5 / 2,7 / 2)-(1,3 / 2,5 / 2)$ & 226874.781 & 16.3 & $1.143 \times 10^{-4}$ & & \\
\hline $\mathrm{CN}$ & $(2,5 / 2,3 / 2)-(1,3 / 2,1 / 2)$ & 226875.896 & 16.3 & $8.587 \times 10^{-5}$ & & \\
\hline $\mathrm{CN}$ & $(2,5 / 2,3 / 2)-(1,3 / 2,3 / 2)$ & 226887.420 & 16.3 & $2.731 \times 10^{-5}$ & & \\
\hline $\mathrm{CN}$ & $(3,5 / 2,7 / 2)-(2,5 / 2,7 / 2)$ & 339516.635 & 32.6 & $2.535 \times 10^{-5}$ & $0.13(0.03)$ & $4.4(0.4)$ \\
\hline $\mathrm{CN}$ & $(3,5 / 2,3 / 2)-(2,3 / 2,3 / 2)$ & 340019.626 & 32.6 & $9.270 \times 10^{-5}$ & \multirow{4}{*}{$1.31(0.05)$} & \\
\hline $\mathrm{CN}$ & $(3,5 / 2,7 / 2)-(2,3 / 2,5 / 2)$ & 340031.549 & 32.6 & $3.845 \times 10^{-4}$ & & $\mathrm{HR}$ \\
\hline $\mathrm{CN}$ & $(3,5 / 2,3 / 2)-(2,3 / 2,1 / 2)$ & 340035.408 & 32.6 & $2.887 \times 10^{-4}$ & & НВ \\
\hline $\mathrm{CN}$ & $(3,5 / 2,5 / 2)-(2,3 / 2,3 / 2)$ & 340035.408 & 32.6 & $\left.3.231 \times 10^{-4}\right)$ & & \\
\hline
\end{tabular}


Table A.1. continued.

\begin{tabular}{|c|c|c|c|c|c|c|}
\hline Molecule & $\begin{array}{l}\text { Transition } \\
\text { QNs }\end{array}$ & $\begin{array}{l}v_{\text {rest }} \\
(\mathrm{MHz})\end{array}$ & $\begin{array}{l}E_{\mathrm{u}} \\
(\mathrm{K})\end{array}$ & $\begin{array}{l}A_{\mathrm{ul}} \\
\left(\mathrm{s}^{-1}\right)\end{array}$ & $\begin{array}{l}\int T_{\text {ant }}^{*} \mathrm{~d} v \\
\left(\mathrm{~K} \mathrm{~km} \mathrm{~s}^{-1}\right)\end{array}$ & $\begin{array}{l}v_{\exp } \\
\left(\mathrm{km} \mathrm{s}^{-1}\right)\end{array}$ \\
\hline $\mathrm{CN}$ & $(3,7 / 2,7 / 2)-(2,5 / 2,5 / 2)$ & 340247.770 & 32.7 & $3.796 \times 10^{-4}$ & \multirow{5}{*}{$0.83(0.05)$} & \multirow{5}{*}{$\mathrm{HB}$} \\
\hline $\mathrm{CN}$ & $(3,7 / 2,9 / 2)-(2,5 / 2,7 / 2)$ & 340247.770 & 32.7 & $4.131 \times 10^{-4}$ & & \\
\hline $\mathrm{CN}$ & $(3,7 / 2,5 / 2)-(2,5 / 2,3 / 2)$ & 340248.544 & 32.7 & $3.674 \times 10^{-4}$ & & \\
\hline $\mathrm{CN}$ & $(3,7 / 2,5 / 2)-(2,5 / 2,5 / 2)$ & 340261.773 & 32.7 & $4.479 \times 10^{-5}$ & & \\
\hline $\mathrm{CN}$ & $(3,7 / 2,7 / 2)-(2,5 / 2,7 / 2)$ & 340264.949 & 32.7 & $\left.3.350 \times 10^{-5}\right)$ & & \\
\hline NS & $\Omega=1 / 2, \mathrm{e},(7 / 2,9 / 2)-(5 / 2,7 / 2)$ & 161297.246 & 16.6 & $6.865 \times 10^{-5}$ & \multirow{3}{*}{$0.35(0.02)$} & \multirow{3}{*}{ HB } \\
\hline NS & $\Omega=1 / 2, \mathrm{e},(7 / 2,7 / 2)-(5 / 2,5 / 2)$ & 161298.411 & 16.6 & $6.301 \times 10^{-5}$ & & \\
\hline NS & $\Omega=1 / 2, \mathrm{e},(7 / 2,5 / 2)-(5 / 2,3 / 2)$ & 161301.747 & 16.6 & $6.108 \times 10^{-5}$ & & \\
\hline NS & $\Omega=1 / 2, \mathrm{f},(7 / 2,9 / 2)-(5 / 2,7 / 2)$ & 161697.257 & 16.7 & $6.916 \times 10^{-5}$ & \multirow{3}{*}{$0.31(0.02)$} & \multirow{3}{*}{ HB } \\
\hline NS & $\Omega=1 / 2, f,(7 / 2,7 / 2)-(5 / 2,5 / 2)$ & 161703.404 & 16.7 & $6.348 \times 10^{-5}$ & & \\
\hline NS & $\Omega=1 / 2, \mathrm{f},(7 / 2,5 / 2)-(5 / 2,3 / 2)$ & 161703.987 & 16.7 & $6.154 \times 10^{-5}$ & & \\
\hline NS & $\Omega=1 / 2, \mathrm{e},(9 / 2,11 / 2)-(7 / 2,9 / 2)$ & 207436.051 & 26.5 & $1.512 \times 10^{-4}$ & \multirow{3}{*}{$0.31(0.03)$} & \multirow{3}{*}{$\mathrm{HB}$} \\
\hline NS & $\Omega=1 / 2, \mathrm{e},(9 / 2,9 / 2)-(7 / 2,7 / 2)$ & 207436.636 & 26.5 & $1.436 \times 10^{-4}$ & & \\
\hline NS & $\Omega=1 / 2, \mathrm{e},(9 / 2,7 / 2)-(7 / 2,5 / 2)$ & 207438.692 & 26.5 & $1.417 \times 10^{-4}$ & & \\
\hline NS & $\Omega=1 / 2, \mathrm{f},(9 / 2,11 / 2)-(7 / 2,9 / 2)$ & 207834.866 & 26.6 & $1.521 \times 10^{-4}$ & \multirow{3}{*}{$0.35(0.03)$} & \multirow{3}{*}{ HB } \\
\hline NS & $\Omega=1 / 2, \mathrm{f},(9 / 2,9 / 2)-(7 / 2,7 / 2)$ & 207838.365 & 26.6 & $1.445 \times 10^{-4}$ & & \\
\hline NS & $\Omega=1 / 2, \mathrm{f},(9 / 2,7 / 2)-(7 / 2,5 / 2)$ & 207838.365 & 26.6 & $1.425 \times 10^{-4}$ & & \\
\hline NS & $\Omega=1 / 2, \mathrm{e},(11 / 2,13 / 2)-(9 / 2,11 / 2)$ & 253570.476 & 38.7 & $2.824 \times 10^{-4}$ & \multirow{4}{*}{$0.47(0.03)$} & \multirow{4}{*}{$\mathrm{HB}$} \\
\hline NS & $\Omega=1 / 2, \mathrm{e},(11 / 2,11 / 2)-(9 / 2,9 / 2)$ & 253570.476 & 38.7 & $2.730 \times 10^{-4}$ & & \\
\hline NS & $\Omega=1 / 2, \mathrm{e},(11 / 2,9 / 2)-(9 / 2,7 / 2)$ & 253572.148 & 38.7 & $2.711 \times 10^{-4}$ & & \\
\hline NS & $\Omega=1 / 2, \mathrm{f},(11 / 2,13 / 2)-(9 / 2,11 / 2)$ & 253968.393 & 38.8 & $2.838 \times 10^{-4}$ & & \\
\hline NS & $\Omega=1 / 2, \mathrm{f},(11 / 2,11 / 2)-(9 / 2,9 / 2)$ & 253970.581 & 38.8 & $2.743 \times 10^{-4}$ & \multirow[t]{2}{*}{$0.42(0.03)$} & \multirow[t]{2}{*}{ HB } \\
\hline NS & $\Omega=1 / 2, \mathrm{f},(11 / 2,9 / 2)-(9 / 2,7 / 2)$ & 253970.581 & 38.8 & $2.723 \times 10^{-4}$ & & \\
\hline NS & $\Omega=1 / 2, \mathrm{f},(15 / 2,17 / 2)-(13 / 2,15 / 2)$ & 346220.137 & 69.8 & $7.385 \times 10^{-4}$ & \multirow{3}{*}{$0.15(0.05)$} & \multirow{3}{*}{$\mathrm{HB}$} \\
\hline NS & $\Omega=1 / 2, \mathrm{f},(15 / 2,15 / 2)-(13 / 2,13 / 2)$ & 346221.163 & 69.8 & $7.250 \times 10^{-4}$ & & \\
\hline NS & $\Omega=1 / 2, \mathrm{f},(15 / 2,13 / 2)-(13 / 2,11 / 2)$ & 346221.163 & 69.8 & $7.234 \times 10^{-4}$ & & \\
\hline
\end{tabular}


L. Velilla Prieto et al.: The millimeter IRAM-30 m line survey toward IK Tauri

Table A.2. Line parameters for transitions that may present variability.

\begin{tabular}{|c|c|c|c|c|c|c|c|}
\hline Molecule & $\begin{array}{l}\text { Transition } \\
\text { QNs }\end{array}$ & $\begin{array}{l}v_{\text {rest }} \\
(\mathrm{MHz})\end{array}$ & $\begin{array}{l}E_{\mathrm{u}} \\
(\mathrm{K}) \\
\end{array}$ & $\begin{array}{l}A_{\mathrm{ul}} \\
\left(\mathrm{s}^{-1}\right)\end{array}$ & $\begin{array}{l}\int T_{\text {ant }}^{*} \mathrm{~d} v \\
\left(\mathrm{~K} \mathrm{~km} \mathrm{~s}^{-1}\right)\end{array}$ & $\begin{array}{l}v_{\exp } \\
\left(\mathrm{km} \mathrm{s}^{-1}\right)\end{array}$ & Julian date \\
\hline $\mathrm{SiO} v=1$ & $2-1$ & 86243.429 & 1775.4 & $2.903 \times 10^{-5}$ & $154.73(0.04)$ & $7(3)$ & 2455018.5 \\
\hline $\mathrm{SiO} v=1$ & $3-2$ & 129363.350 & 1781.6 & $1.050 \times 10^{-4}$ & $50.01(0.02)$ & $5(2)$ & 2455018.5 \\
\hline $\mathrm{SiO} v=1$ & $4-3$ & 172481.120 & 1789.9 & $2.580 \times 10^{-4}$ & $12.98(0.02)$ & $5(2)$ & 2455578.5 \\
\hline $\mathrm{SiO} v=1$ & $5-4$ & 215596.021 & 1800.3 & $5.154 \times 10^{-4}$ & $7.52(0.03)$ & $4(1)$ & 2456579.5 \\
\hline $\mathrm{SiO} v=1$ & $6-5$ & 258707.335 & 1812.7 & $9.042 \times 10^{-4}$ & $1.04(0.07)$ & $8(1)$ & 2455309.5 \\
\hline$\ldots$ & $\ldots$ & $\ldots$ & $\ldots$ & $\ldots$ & $1.99(0.05)$ & $4(1)$ & 2456579.5 \\
\hline $\mathrm{SiO} v=1$ & $7-6$ & 301814.346 & 1827.2 & $1.452 \times 10^{-3}$ & $4.40(0.08)$ & $6(1)$ & 2455550.5 \\
\hline$\ldots$ & $\ldots$ & $\ldots$ & $\ldots$ & $\ldots$ & $5.35(0.10)$ & $6(1)$ & 2455574.5 \\
\hline$\ldots$ & $\ldots$ & & & $\ldots$ & $4.88(0.05)$ & $8(1)$ & 2456320.5 \\
\hline$\ldots$ & $\ldots$ & $\ldots$ & $\ldots$ & $\ldots$ & $2.33(0.12)$ & $10(1)$ & 2456616.5 \\
\hline $\mathrm{SiO} v=1$ & $8-7$ & 344916.336 & 1843.7 & $2.185 \times 10^{-3}$ & $0.74(0.08)$ & $8(1)$ & 2455576.5 \\
\hline $\mathrm{SiO} v=2$ & $2-1$ & 85640.455 & 3527.5 & $2.879 \times 10^{-5}$ & $0.77(0.02)$ & $7(3)$ & 2455018.5 \\
\hline $\mathrm{SiO} v=2$ & $3-2$ & 128458.888 & 3533.6 & $1.041 \times 10^{-4}$ & $1.63(0.01)$ & $4.70(2)$ & 2455018.5 \\
\hline $\mathrm{SiO} v=2$ & $4-3$ & 171275.169 & 3541.9 & $2.559 \times 10^{-4}$ & $6.62(0.04)$ & $8(2)$ & 2455578.5 \\
\hline $\mathrm{SiO} v=2$ & $5-4$ & 214088.579 & 3552.1 & $5.110 \times 10^{-4}$ & $1.94(0.03)$ & $7(1)$ & 2456579.5 \\
\hline $\mathrm{SiO} v=2$ & $6-5$ & 256898.401 & 3564.5 & $8.966 \times 10^{-4}$ & $2.03(0.05)$ & $7(1)$ & 2456579.5 \\
\hline $\mathrm{SiO} v=2$ & $7-6$ & 299703.918 & 3578.9 & $1.439 \times 10^{-3}$ & $1.63(0.09)$ & $2(1)$ & 2455550.5 \\
\hline$\ldots$ & $\ldots$ & $\ldots$ & $\ldots$ & $\ldots$ & $6.29(0.06)$ & $3(1)$ & 2456320.5 \\
\hline $\mathrm{SiO} v=2$ & $8-7$ & 342504.411 & 3595.3 & $2.166 \times 10^{-3}$ & $0.34(0.07)$ & $5,(1)$ & 2455576.5 \\
\hline $\mathrm{SiO} v=3$ & $4-3$ & 170070.353 & 5276.8 & $2.536 \times 10^{-4}$ & $2.78(0.02)$ & $4(2)$ & 2455576.5 \\
\hline $\mathrm{SiO} v=3$ & $5-4$ & 212582.557 & 5287.0 & $5.066 \times 10^{-4}$ & $0.12(0.03)$ & $3(1)$ & 2456579.5 \\
\hline $\mathrm{SiO} v=3$ & $6-5$ & 255091.170 & 5299.2 & $8.888 \times 10^{-4}$ & $0.78(0.03)$ & $8(1)$ & 2456579.5 \\
\hline $\mathrm{SiO} v=3$ & $7-6$ & 297595.476 & 5313.5 & $1.427 \times 10^{-3}$ & $1.46(0.03)$ & $2(3)$ & 2456371.5 \\
\hline$\ldots$ & $\ldots$ & $\ldots$ & $\ldots$ & $\ldots$ & $0.01(0.02)$ & $3(1)$ & 2456615.5 \\
\hline $\mathrm{SiO} v=3$ & $8-7$ & 340094.756 & 5329.8 & $2.148 \times 10^{-3}$ & $0.36(0.05)$ & $5(1)$ & 2455575.5 \\
\hline$\ldots$ & $\ldots$ & $\ldots$ & $\ldots$ & $\ldots$ & $0.37(0.10)$ & $3(1)$ & 2456615.5 \\
\hline${ }^{29} \mathrm{SiO} v=1$ & $2-1$ & 85166.957 & 1764.3 & $2.796 \times 10^{-5}$ & $1.50(0.03)$ & $7(3)$ & 2455018.5 \\
\hline${ }^{29} \mathrm{SiO} v=1$ & $4-3$ & 170328.320 & 1778.6 & $2.485 \times 10^{-4}$ & $9.34(0.02)$ & $5(2)$ & 2455576.5 \\
\hline${ }^{29} \mathrm{SiO} v=1$ & $5-4$ & 212905.155 & 1788.8 & $4.963 \times 10^{-4}$ & $0.15(0.03)$ & $1(2)$ & 2456579.5 \\
\hline${ }^{29} \mathrm{SiO} v=1$ & $6-5$ & 255478.493 & 1801.1 & $8.707 \times 10^{-4}$ & $11.18(0.04)$ & $5(2)$ & 2456579.5 \\
\hline${ }^{29} \mathrm{SiO} v=1$ & $7-6$ & 298047.635 & 1815.4 & $1.398 \times 10^{-3}$ & $0.80(0.06)$ & $5(1)$ & 2455550.5 \\
\hline$\ldots$ & $\ldots$ & $\ldots$ & $\ldots$ & $\ldots$ & $2.91(0.04)$ & $4(1)$ & 2456320.5 \\
\hline$\ldots$ & $\ldots$ & $\ldots$ & $\ldots$ & $\ldots$ & $2.21(0.04)$ & $5(1)$ & 2456371.5 \\
\hline & $\ldots$ & $\ldots$ & & $\ldots$ & $0.35(0.03)$ & $4(1)$ & 2456615.5 \\
\hline${ }^{29} \mathrm{SiO} v=1$ & $8-7$ & 340611.882 & 1831.8 & $2.104 \times 10^{-3}$ & $0.21(0.06)$ & $5(1)$ & 2455575.5 \\
\hline${ }^{29} \mathrm{SiO} v=2$ & $2-1$ & 84575.290 & 3505.6 & $2.773 \times 10^{-5}$ & $12.64(0.02)$ & $10(3)$ & 2455018.5 \\
\hline${ }^{29} \mathrm{SiO} v=2$ & $4-3$ & 169144.982 & 3519.8 & $2.464 \times 10^{-4}$ & $0.66(0.02)$ & $6(2)$ & 2455576.5 \\
\hline${ }^{29} \mathrm{SiO} v=2$ & $5-4$ & 211425.980 & 3529.9 & $4.922 \times 10^{-4}$ & $0.16(0.02)$ & $2(2)$ & 2456579.5 \\
\hline${ }^{29} \mathrm{SiO} v=2$ & $6-5$ & 253703.479 & 3542.1 & $8.635 \times 10^{-4}$ & $9.61(0.03)$ & $1(2)$ & 2456579.5 \\
\hline${ }^{29} \mathrm{SiO} v=3$ & $8-7$ & 335880.698 & 5296.5 & $2.069 \times 10^{-3}$ & $0.3(0.3)$ & $8(2)$ & 2455579.5 \\
\hline$\ldots$ & $\ldots$ & $\ldots$ & $\ldots$ & $\ldots$ & $1.0(0.2)$ & $2(2)$ & 2456371.5 \\
\hline$\ldots$ & $\ldots$ & $\ldots$ & $\ldots$ & $\ldots$ & $0.57(0.10)$ & $2(2)$ & 2456615.5 \\
\hline${ }^{30} \mathrm{SiO} v=1$ & $4-3$ & 168323.349 & 1768.0 & $2.398 \times 10^{-4}$ & $2.00(0.02)$ & $4(5)$ & 2455576.5 \\
\hline${ }^{30} \mathrm{SiO} v=1$ & $5-4$ & 210399.065 & 1778.1 & $4.790 \times 10^{-4}$ & $1.11(0.04)$ & $6(2)$ & 2456579.5 \\
\hline${ }^{30} \mathrm{SiO} v=1$ & $6-5$ & 252471.366 & 1790.3 & $8.403 \times 10^{-4}$ & $0.15(0.04)$ & $4(2)$ & 2456579.5 \\
\hline & $\ldots$ & $\ldots$ & $\ldots$ & $\ldots$ & $0.08(0.03)$ & $4(2)$ & 2456579.5 \\
\hline${ }^{30} \mathrm{SiO} v=1$ & $7-6$ & 294539.570 & 1804.4 & $1.349 \times 10^{-3}$ & $0.59(0.04)$ & $6(2)$ & 2456320.5 \\
\hline$\ldots$ & $\ldots$ & $\ldots$ & & $\ldots$ & $0.35(0.04)$ & $5(1)$ & 2456371.5 \\
\hline$\ldots$ & $\ldots$ & $\ldots$ & & $\ldots$ & $0.23(0.03)$ & $6(1)$ & 2456615.5 \\
\hline
\end{tabular}

Notes. Column 2: quantum numbers of the corresponding line; Col. 6: integrated intensity (in antenna temperature) of the line with its formal uncertainty; Col. 7: expansion velocity derived from the linewidth with its uncertainty which includes the width of half a channel (1 MHz) except for the lines with a low $\mathrm{S} / \mathrm{N}$ where a full channel $(2 \mathrm{MHz})$ is included. Column 8: Julian date. The uncertainties given (within parentheses) do not include additional uncertainties derived from the absolute flux calibration and baseline subtraction. The ellipsis symbol indicate that the field is equal to the same field of the previous row. 
Table A.2. continued.

\begin{tabular}{|c|c|c|c|c|c|c|c|}
\hline Molecule & $\begin{array}{l}\text { Transition } \\
\text { QNs }\end{array}$ & $\begin{array}{l}v_{\text {rest }} \\
(\mathrm{MHz})\end{array}$ & $\begin{array}{l}E_{\mathrm{u}} \\
(\mathrm{K})\end{array}$ & $\begin{array}{l}A_{\mathrm{ul}} \\
\left(\mathrm{s}^{-1}\right)\end{array}$ & $\begin{array}{l}\int T_{\text {ant }}^{*} \mathrm{~d} v \\
\left(\mathrm{~K} \mathrm{~km} \mathrm{~s}^{-1}\right)\end{array}$ & $\begin{array}{l}v_{\exp } \\
\left(\mathrm{km} \mathrm{s}^{-1}\right)\end{array}$ & Julian date \\
\hline \multirow{5}{*}{$\begin{array}{l}{ }^{30} \mathrm{SiO} v=1 \\
\ldots\end{array}$} & $8-7$ & 336602.993 & 1820.6 & $2.031 \times 10^{-3}$ & $2.8(0.3)$ & $9(1)$ & 2456371.5 \\
\hline & & & & $\ldots$ & $3.3(0.3)$ & & \\
\hline & & $\ldots$ & & & $7.14(0.11)$ & $6(1)$ & 2456615.5 \\
\hline & & $\ldots$ & & . & $7.77(0.13)$ & & 2456615.5 \\
\hline & $\ldots$ & $\ldots$ & & $\ldots$ & $3.5(0.3)$ & $6(1)$ & 2455579.5 \\
\hline \multirow{2}{*}{$\begin{array}{l}{ }^{30} \mathrm{SiO} v=2 \\
{ }^{30} \mathrm{SiO} v=2\end{array}$} & $2-1$ & 83583.203 & 3485.0 & $2.676 \times 10^{-5}$ & $0.45(0.02)$ & $7(7)$ & 2455018.5 \\
\hline & $4-3$ & 167160.941 & 3499.1 & $2.379 \times 10^{-4}$ & $0.68(0.01)$ & $4(3)$ & 575.5 \\
\hline \multirow{6}{*}{$\begin{array}{l}\text { SiS } v=1 \\
\text { SiS } v=1 \\
\text { SiS } v=1 \\
\text { SiS } v=1 \\
\text { SiS } v=1\end{array}$} & $8-7$ & 144520.367 & 1102.4 & $5.034 \times 10^{-5}$ & $0.04(0.01)$ & $19(5)$ & 018.5 \\
\hline & -8 & 81.756 & & $7.215 \times 10^{-5}$ & 0.13 & & 50.5 \\
\hline & $2-11$ & 216757.603 & 1138.8 & $1.733 \times 10^{-4}$ & $0.10(0.03)$ & $4(3)$ & 579.5 \\
\hline & $13-12$ & 234812.968 & 1150.1 & $2.209 \times 10^{-4}$ & $0.16(0 . c$ & & 79.5 \\
\hline & $14-13$ & 252866.469 & 1162.2 & $2.766 \times 10^{-4}$ & $0.14(C$ & 7( & 79.5 \\
\hline & & & & & $0.14(0.02)$ & 5( & 579.5 \\
\hline \multirow{3}{*}{$\begin{array}{l}\operatorname{SiS} v=1 \\
\operatorname{SiS} v=1 \\
\operatorname{SiS} v=1\end{array}$} & $5-14$ & 270917.961 & 1175.2 & $3.410 \times 10^{-4}$ & $0.13(0.04)$ & 4 & 309.5 \\
\hline & $17-16$ & 307014.349 & 1203.8 & $4.982 \times 10^{-4}$ & $0.32(0.06)$ & $5(2)$ & 320.5 \\
\hline & $19-18$ & 343100.984 & 1235.9 & $6.974 \times 10^{-4}$ & $0.30(0.05)$ & $9(2)$ & 2455576.5 \\
\hline $\mathrm{o}-\mathrm{H}_{2} \mathrm{O}$ & $10_{2,9}-9_{3,6}$ & 321225.640 & 1827.1 & $6.124 \times 10^{-6}$ & $1.0(0.2)$ & $5(2)$ & 2455574.5 \\
\hline \multirow{4}{*}{$\begin{array}{l}\mathrm{o}-\mathrm{H}_{2} \mathrm{O} v_{2}=1 \\
\mathrm{o}-\mathrm{H}_{2} \mathrm{O} v_{2}=1 \\
\ldots\end{array}$} & & 232686.700 & & $4.630 \times 10^{-6}$ & $0.18(0.03)$ & & 2456579.5 \\
\hline & $6_{6,1}-7_{5,2}$ & 293664.442 & 3899.5 & $7.018 \times 10^{-6}$ & $0.48(0.04)$ & 5( & 320.5 \\
\hline & $\ldots$ & $\ldots$ & & & $0.26(0.04)$ & $4(2)$ & 2456371.5 \\
\hline & $\ldots$ & $\ldots$ & & $\cdots$ & $0.22(0.04)$ & $8(2)$ & 2456615.5 \\
\hline \multirow{3}{*}{$\begin{array}{l}\mathrm{p}-\mathrm{H}_{2} \mathrm{O} v_{2}=1 \\
\mathrm{p}-\mathrm{H}_{2} \mathrm{O} v_{2}=1 \\
\ldots\end{array}$} & $4_{4,0}-5_{3,3}$ & 96259.644 & 3064.2 & & $0.10(0.01)$ & $7(5)$ & 2455019.5 \\
\hline & $5_{5,1}-6_{4,2}$ & 209118.370 & 3462.1 & $3.369 \times 10^{-6}$ & $0.21(0.03)$ & $7(2)$ & 372.5 \\
\hline & $\ldots$ & thes & & & $0.06(0.06)$ & $8(2)$ & 2456579.5 \\
\hline \multirow{2}{*}{$\begin{array}{l}\mathrm{p}-\mathrm{H}_{2} \mathrm{O} v_{2}=1 \\
\ldots\end{array}$} & $6_{6,0}-7_{5,3}$ & 297439.107 & 3933.7 & $7.289 \times 10^{-6}$ & $0.12(0.04)$ & $5(2)$ & 2456320.5 \\
\hline & $\ldots$ & $\ldots$ & $\ldots$ & $\ldots$ & $0.17(0.03)$ & $7(2)$ & 2456371.5 \\
\hline$\ldots$ & $\ldots$ & $\ldots$ & & $\ldots$ & $0.08(0.03)$ & $5(2)$ & 2456615.5 \\
\hline \multirow{2}{*}{$\begin{array}{l}\mathrm{o}-\mathrm{H}_{2} \mathrm{O} v_{2}=2 \\
\ldots\end{array}$} & $6_{5,2}-7_{4,3}$ & 268149.175 & 6005.0 & $1.471 \times 10^{-5}$ & $2.38(0.07)$ & $2(1)$ & 2455309.5 \\
\hline & $\ldots$ & $\ldots$ & $\ldots$ & $\ldots$ & $0.12(0.03)$ & $7(1)$ & 2456579.5 \\
\hline $\mathrm{p}-\mathrm{H}_{2} \mathrm{O} v_{2}=2$ & $3_{3,1}-4_{2,2}$ & 137048.521 & 5036.7 & $2.096 \times 10^{-6}$ & $0.03(0.01)$ & $8(5)$ & 2455019.5 \\
\hline
\end{tabular}




\section{Appendix B: Unidentified lines}

Table B.1. Unidentified features and, in some cases, tentative line identification.

\begin{tabular}{|c|c|c|c|}
\hline $\begin{array}{l}v_{\text {rest }} \\
(\mathrm{MHz})\end{array}$ & $\begin{array}{l}\text { Tentative assignment } \\
-\end{array}$ & $\begin{array}{l}\int T_{\text {ant }}^{*} \mathrm{~d} v \\
\left(\mathrm{~K} \mathrm{~km} \mathrm{~s}^{-1}\right)\end{array}$ & $\begin{array}{l}v_{\exp } \\
\left(\mathrm{km} \mathrm{s}^{-1}\right)\end{array}$ \\
\hline 81166 & - & $0.08(0.03)$ & $16(7)$ \\
\hline 83825 & $\mathrm{SiO} v=5 J=2-1$ & $0.30(0.03)$ & $35(5)$ \\
\hline 84478 & - & $0.06(0.02)$ & $7(7)$ \\
\hline 86190 & $\mathrm{OH} v=1 J, \Omega, p, F=8 / 2,3 / 2,1,9-8 / 2,3 / 2,-1,9$ & $0.71(0.02)$ & $7(3)$ \\
\hline 87385 & - & $0.23(0.02)$ & $17(3)$ \\
\hline 108892 & - & $0.09(0.01)$ & $11(4)$ \\
\hline 128883 & $\mathrm{TiO}_{2} J_{\mathrm{K}, \mathrm{k}}=19_{10,10}-20_{9,11}$ & $0.10(0.02)$ & $13(4)$ \\
\hline 131195 & $\operatorname{SiN} N, J, F=3,7 / 2,7 / 2-2,5 / 2,7 / 2$ & $0.05(0.01)$ & $7(4)$ \\
\hline 136946 & - & $0.15(0.01)$ & $27(4)$ \\
\hline 139065 & - & $0.05(0.01)$ & $13(4)$ \\
\hline 143997 & - & $0.05(0.01)$ & $7(4)$ \\
\hline 145101 & $\mathrm{~N}^{17} \mathrm{O} J=3 / 2-1 / 2$ hfs blend & $0.11(0.02)$ & $18(4)$ \\
\hline 149968 & - & $0.12(0.02)$ & $18(4)$ \\
\hline 150165 & - & $0.13(0.03)$ & $20(4)$ \\
\hline 158409 & ${ }^{29} \mathrm{Si}^{18} \mathrm{O} J=4-3$ & $0.04(0.01)$ & $10(4)$ \\
\hline 159227 & ${ }^{29} \mathrm{SiS} v=1 J=9-8$ & $0.08(0.01)$ & $13(4)$ \\
\hline 159607 & - & $0.08(0.01)$ & $4(3)$ \\
\hline 161197 & - & $0.06(0.02)$ & $8(4)$ \\
\hline 165641 & $\mathrm{Na}^{37} \mathrm{Cl} J=13-12$ & $0.09(0.01)$ & $11(4)$ \\
\hline 169652 & - & $0.12(0.02)$ & $16(3)$ \\
\hline 172925 & - & $0.47(0.04)$ & $28(3)$ \\
\hline 208612 & $\mathrm{TiO}_{2} J_{\mathrm{K}, \mathrm{k}}=43_{10,34}-42_{11,31}$ & $0.09(0.02)$ & $3(3)$ \\
\hline 209295 & - & $0.22(0.03)$ & $17(2)$ \\
\hline 216602 & ${ }^{13} \mathrm{CN} J=2-1 \mathrm{hfs}$ blend & $0.26(0.03)$ & $8(2)$ \\
\hline 218065 & - & $0.41(0.02)$ & $3(3)$ \\
\hline 218575 & - & $0.43(0.03)$ & $24(2)$ \\
\hline 219840 & ${ }^{34} \mathrm{SO}_{2} J_{\mathrm{K}, \mathrm{k}}=64,2-73,5$ & $0.35(0.04)$ & $3(2)$ \\
\hline 221614 & - & $0.48(0.04)$ & $18(2)$ \\
\hline 223370 & $\mathrm{SO}^{18} \mathrm{O} J_{\mathrm{K}, \mathrm{k}}=6_{4,2}-7_{3,5}$ & $0.35(0.05)$ & $22(3)$ \\
\hline 224876 & - & $1.98(0.06)$ & $17(2)$ \\
\hline 250807 & - & $0.15(0.02)$ & $8(3)$ \\
\hline 252256 & - & $0.20(0.02)$ & $19(3)$ \\
\hline 252531 & - & $0.45(0.04)$ & $15(3)$ \\
\hline 254677 & - & $0.34(0.03)$ & $25(3)$ \\
\hline 263452 & - & $0.12(0.03)$ & $4(2)$ \\
\hline 271968 & - & $0.44(0.05)$ & $7(2)$ \\
\hline 306192 & $\mathrm{AlO} N, J, F=8,15 / 2,8-7,15 / 2,7$ & $0.64(0.08)$ & $22(2)$ \\
\hline
\end{tabular}

Notes. Column 3: integrated intensity (in antenna temperature) of the line with its formal uncertainty; Col. 4: expansion velocity derived from the linewidth with its uncertainty which includes the width of half a channel $(1 \mathrm{MHz})$ except for the lines with a low $\mathrm{S} / \mathrm{N}$ where a full channel $(2 \mathrm{MHz})$ is included. The uncertainties given (within parentheses) do not include additional uncertainties derived from the absolute flux calibration and baseline subtraction. 
Table B.2. Artifacts identified in the spectra between $167475-174800 \mathrm{MHz}$.

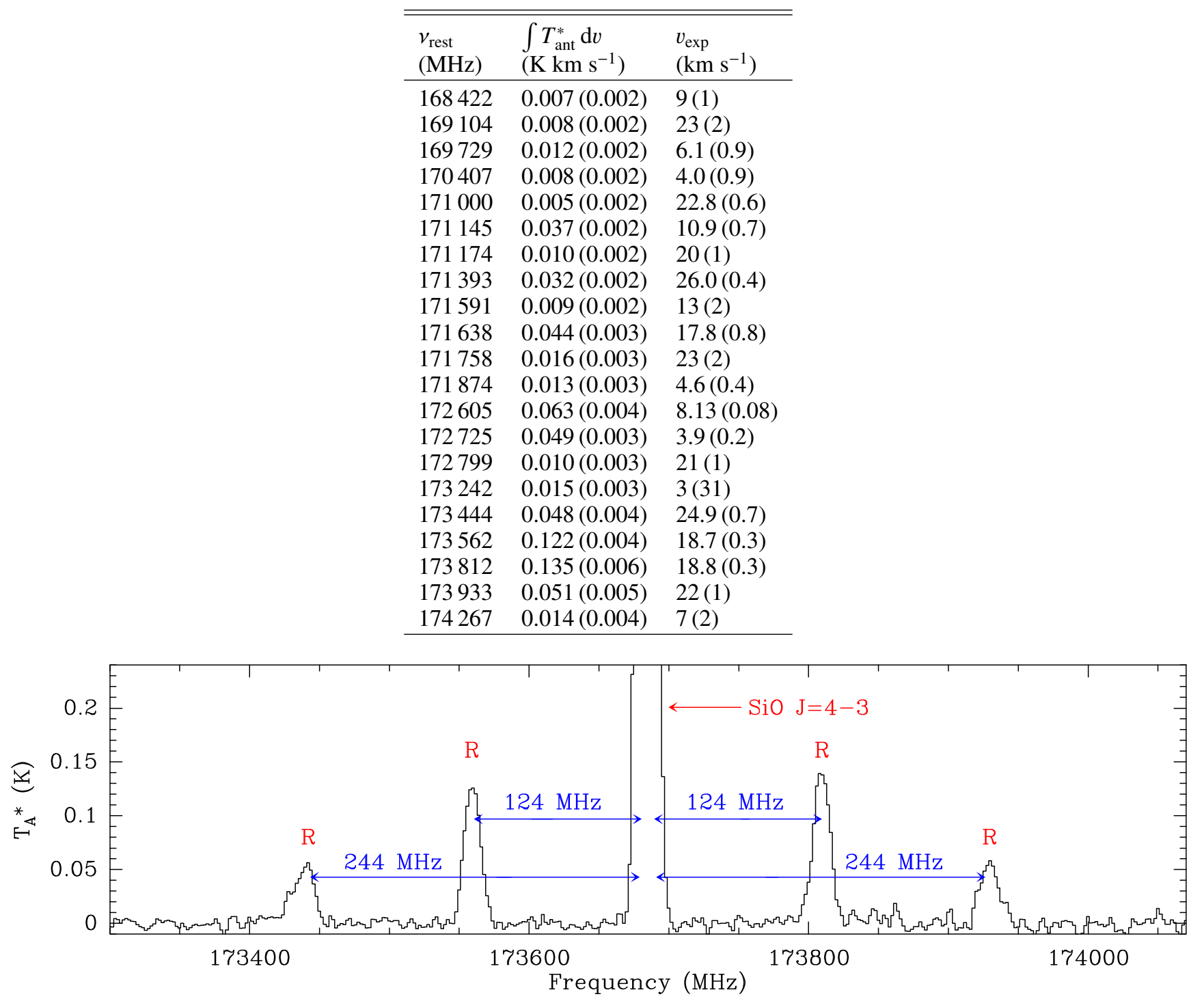

Fig. B.1. Example of the artifacts $(R)$ produced by the receivers (see Table B.2). Several symmetrical spurious replicas of the real SiO $J=4-3$ line appear at both sides of it (equidistant). The intensities of the replicas decrease with the frequency distance to the real feature. 
L. Velilla Prieto et al.: The millimeter IRAM-30 m line survey toward IK Tauri

\section{Appendix C: IRAM-30 m survey of IK Tau}
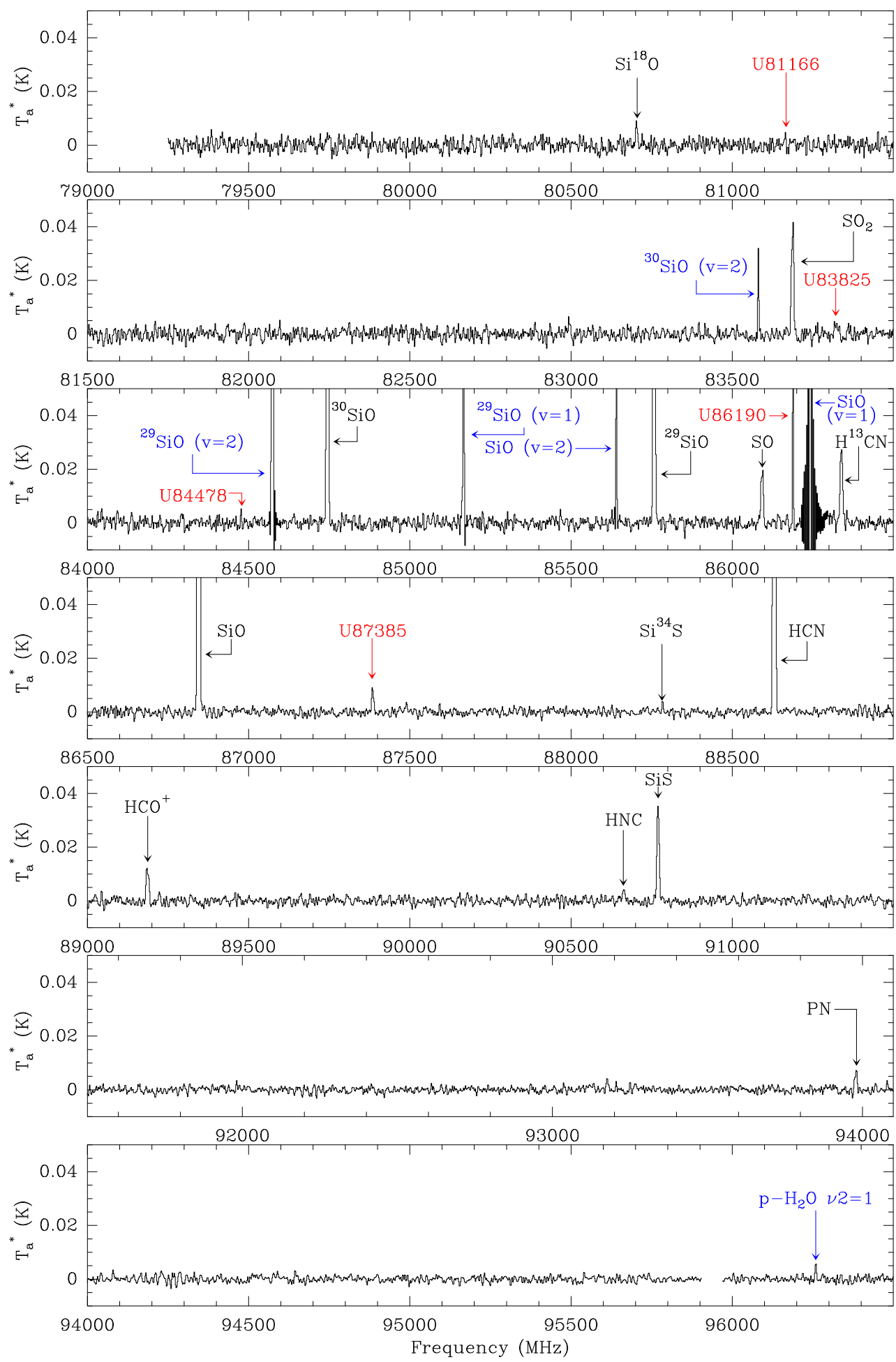

Fig. C.1. Line identification of the IRAM-30 m line survey of IK Tau. We marked in red the unidentified lines (UIs) with the central frequency of the line. The lines which display time variability of their intensity are marked in blue. Image band and spurious feaures/artifacts have been blanked off (see Table B.2 and Fig. B.1) 
A\&A 597, A25 (2017)
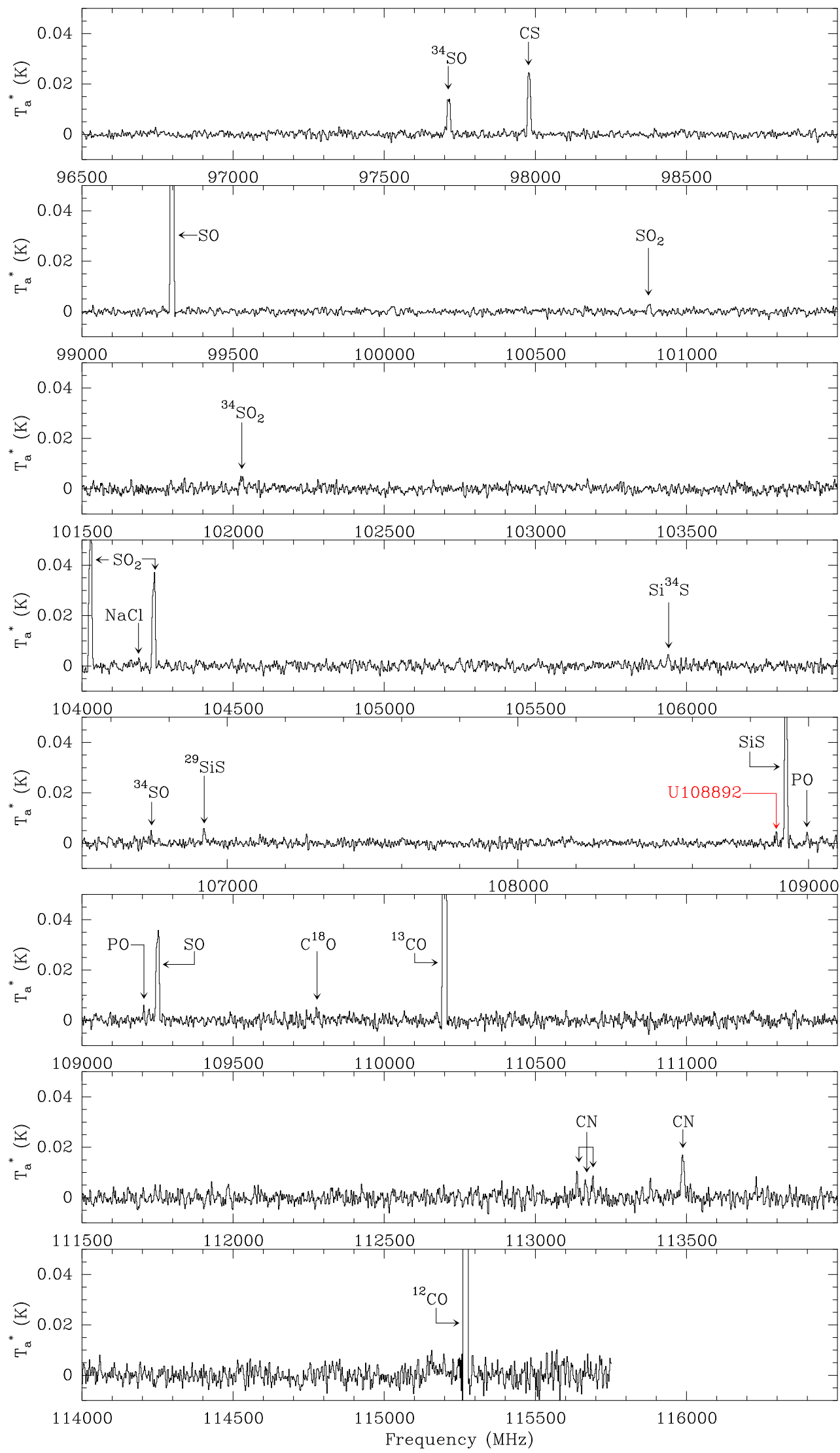

Fig. C.1. continued. 
L. Velilla Prieto et al.: The millimeter IRAM-30 m line survey toward IK Tauri
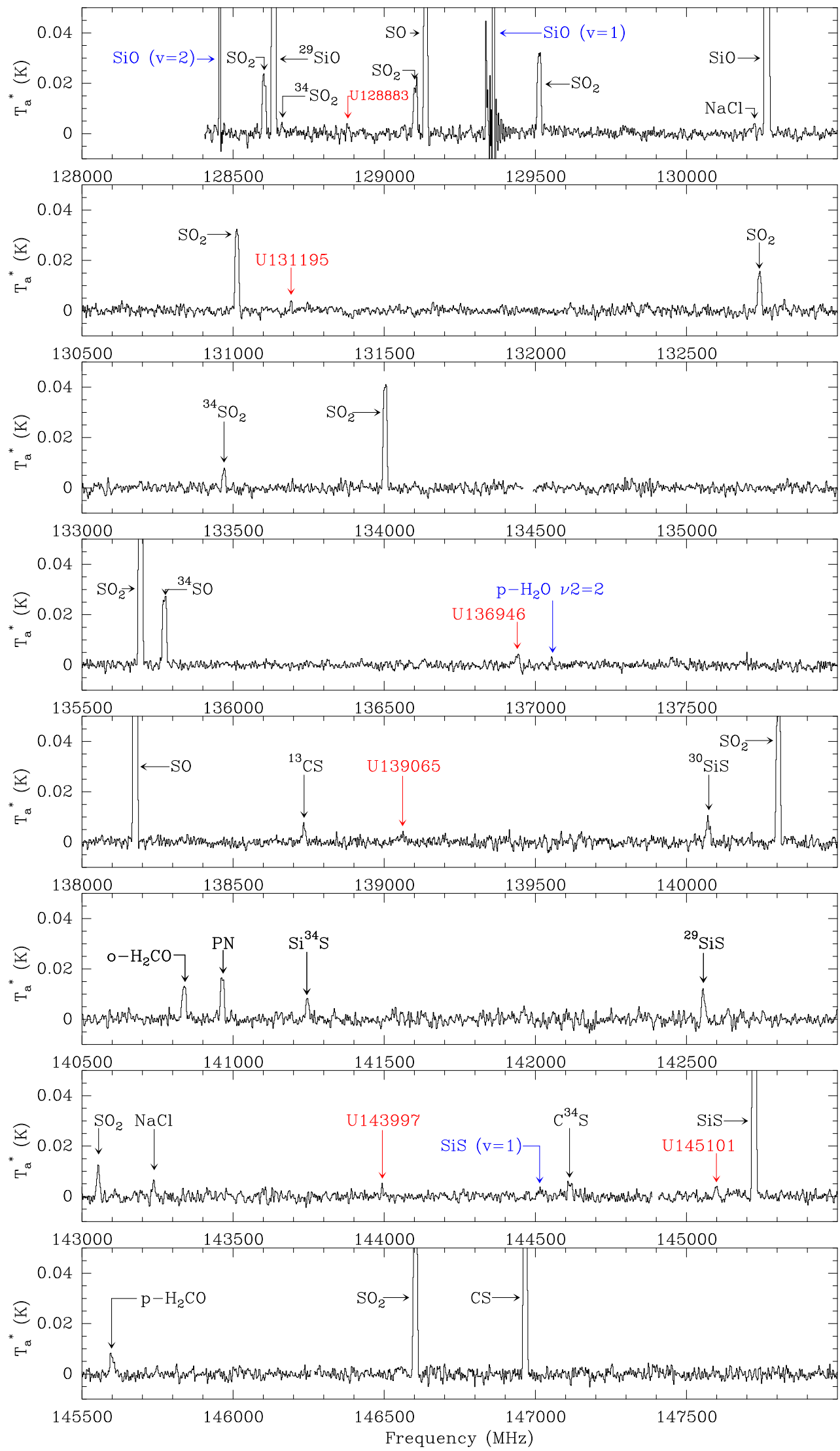

Fig. C.1. continued. 
A\&A 597, A25 (2017)
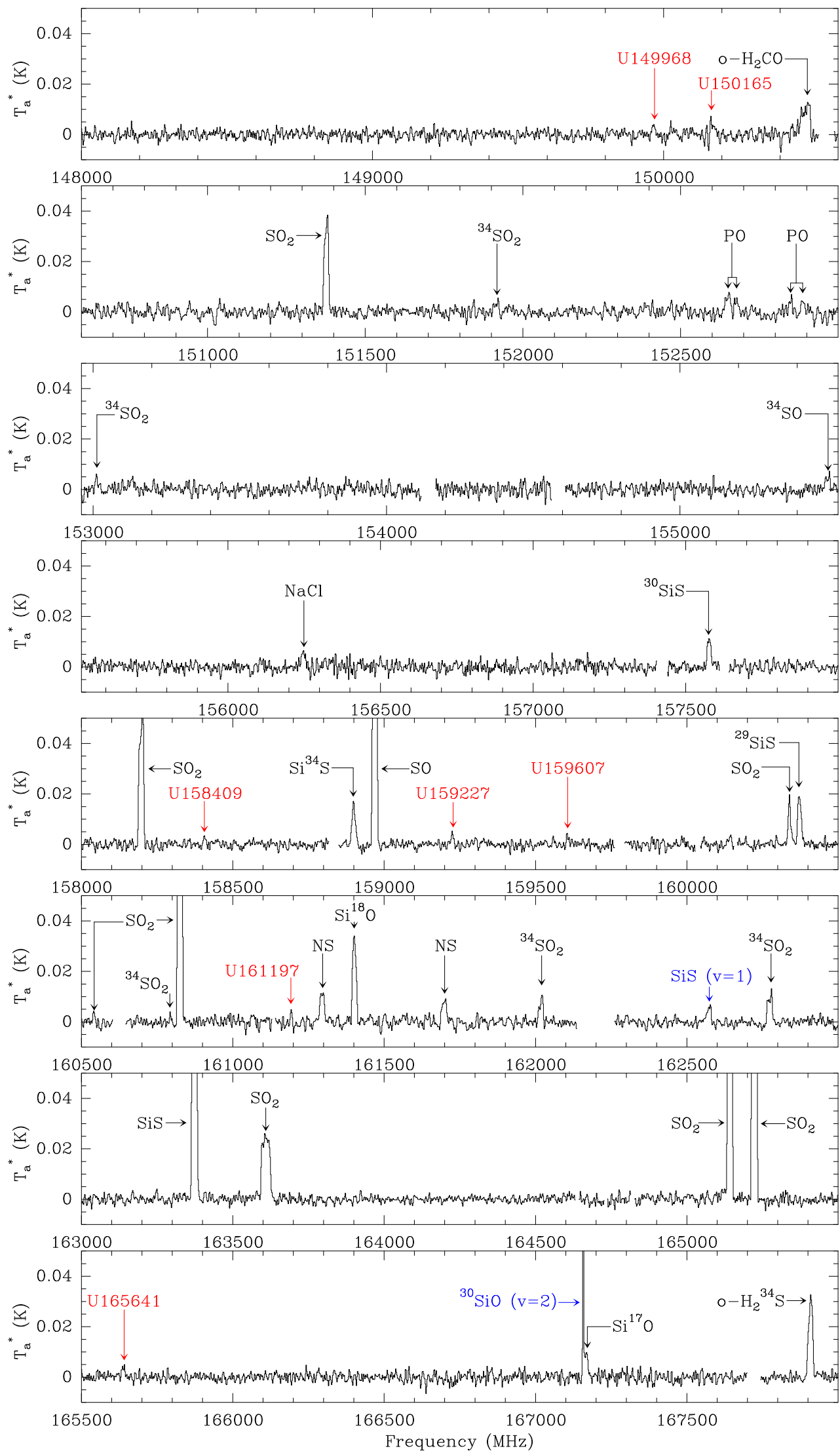

Fig. C.1. continued. 
L. Velilla Prieto et al.: The millimeter IRAM-30 m line survey toward IK Tauri
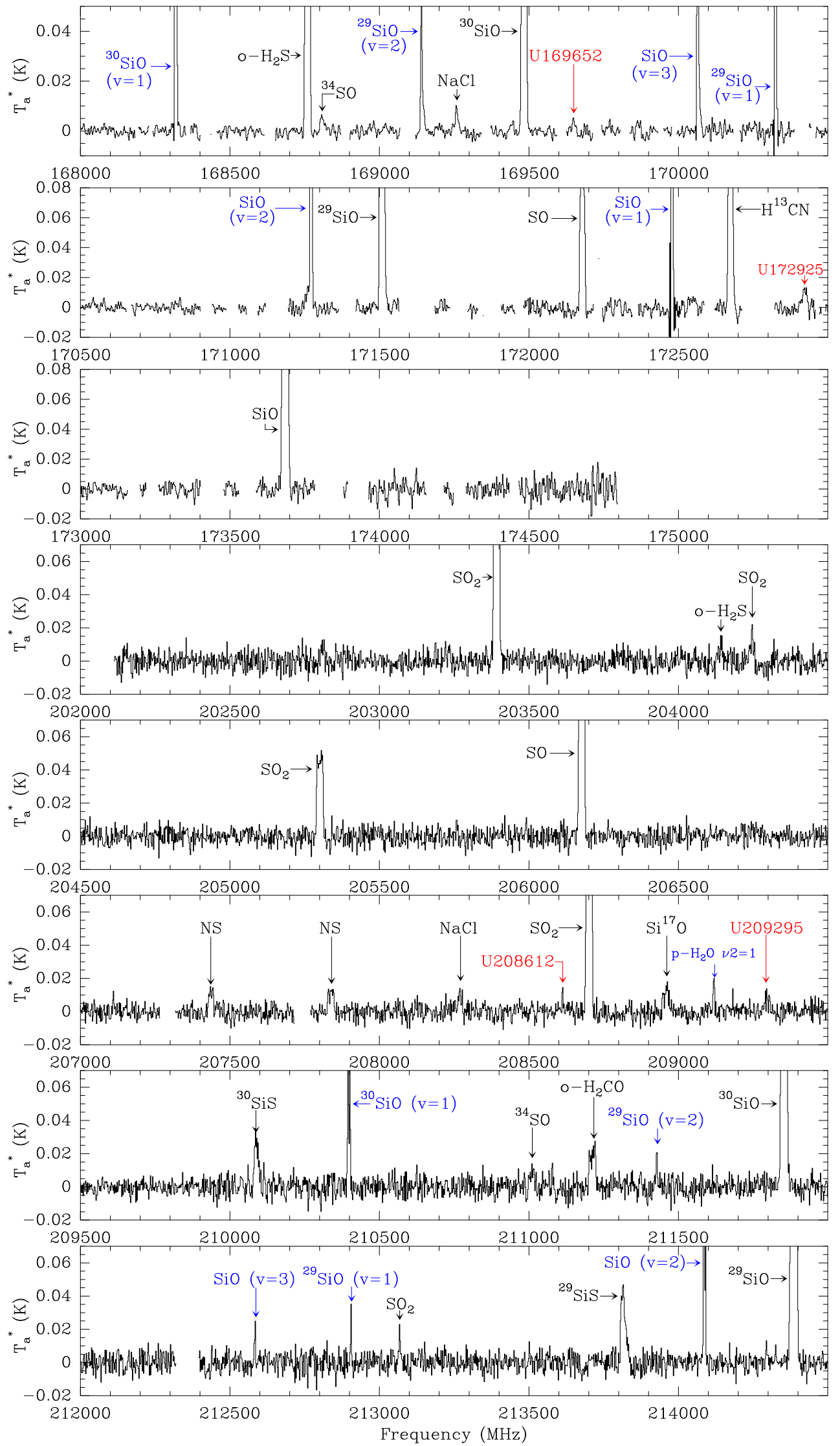

Fig. C.1. continued. 
A\&A 597, A25 (2017)
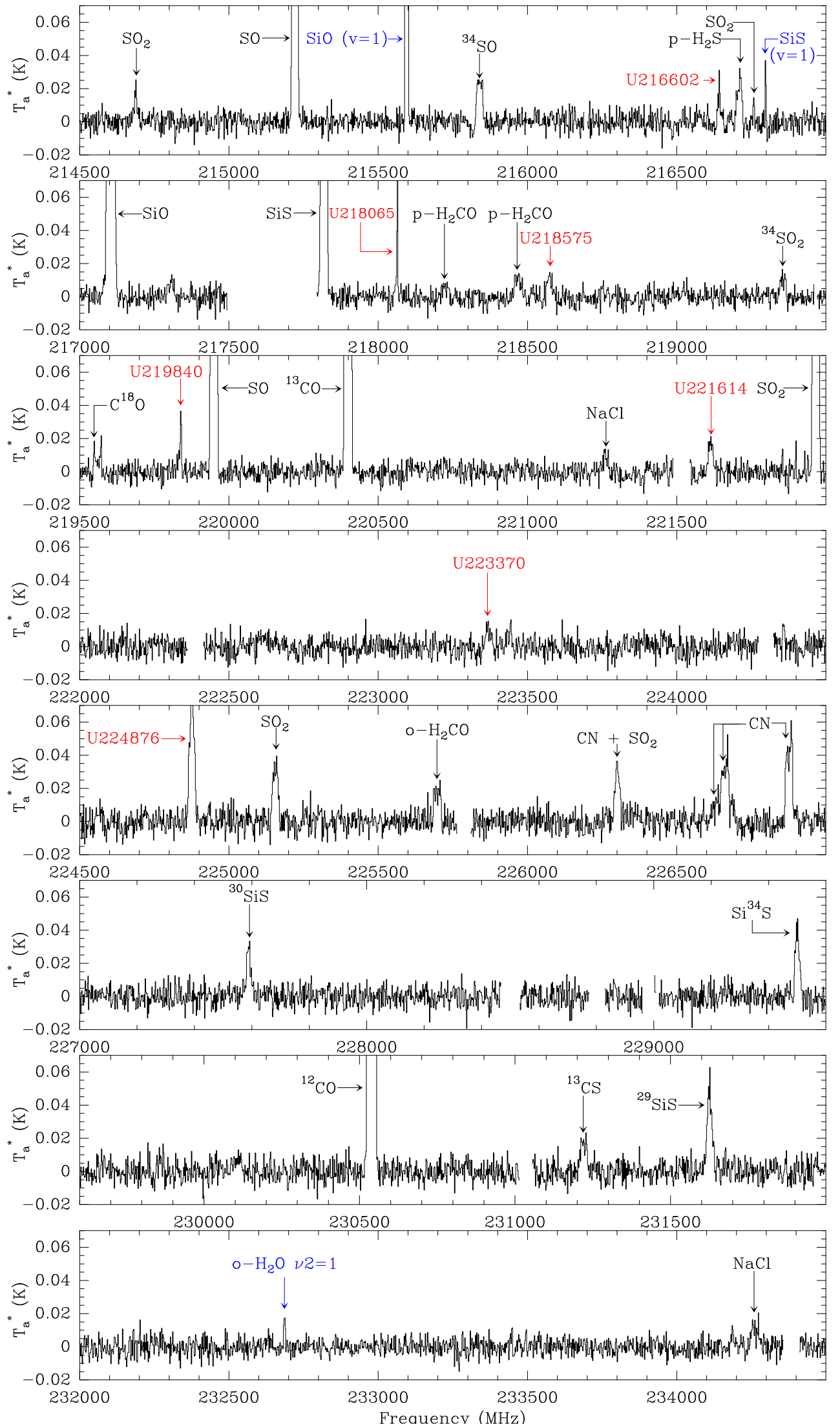

Fig. C.1. continued. 
L. Velilla Prieto et al.: The millimeter IRAM-30 m line survey toward IK Tauri
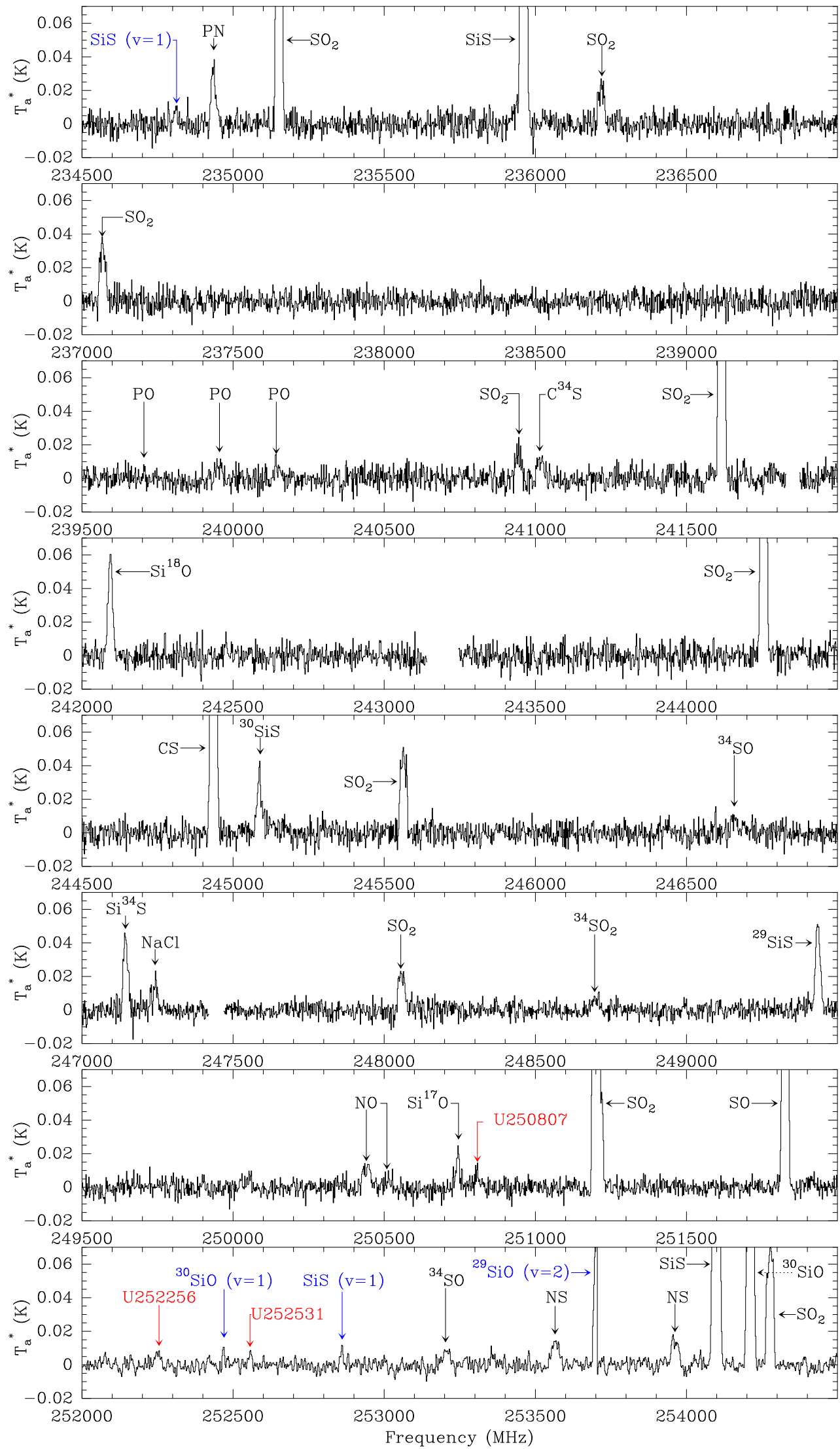

Fig. C.1. continued. 
A\&A 597, A25 (2017)

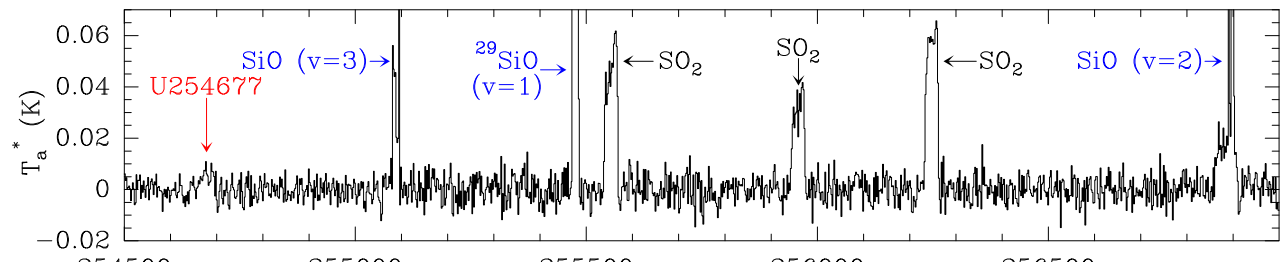

0.06
0.04
0.02

$=50$.

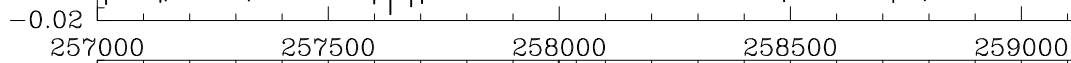

$0.04=2$

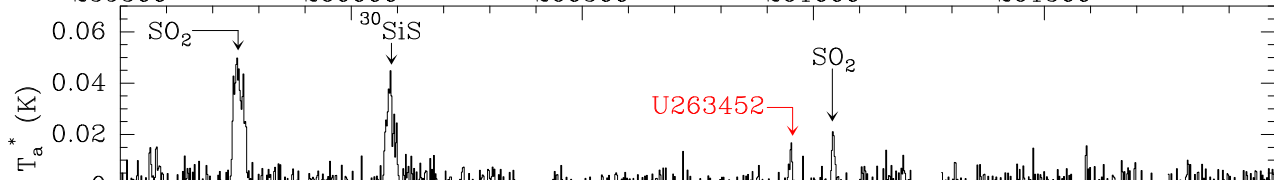

- 1 .

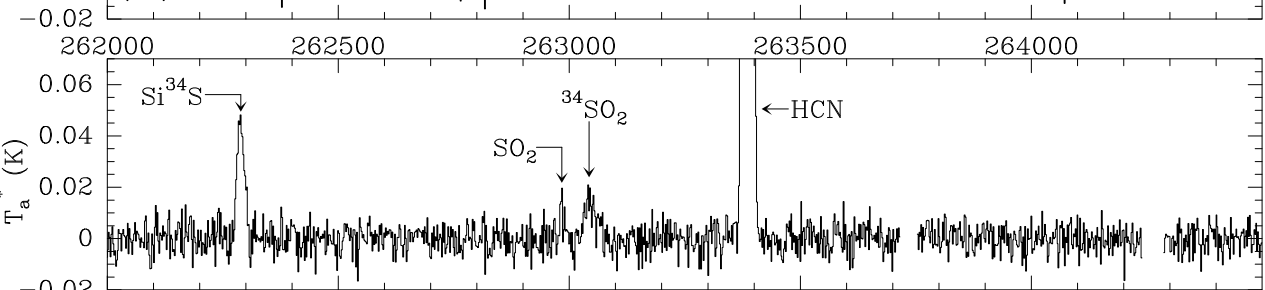

$264500,265000,265500,260000,26000$

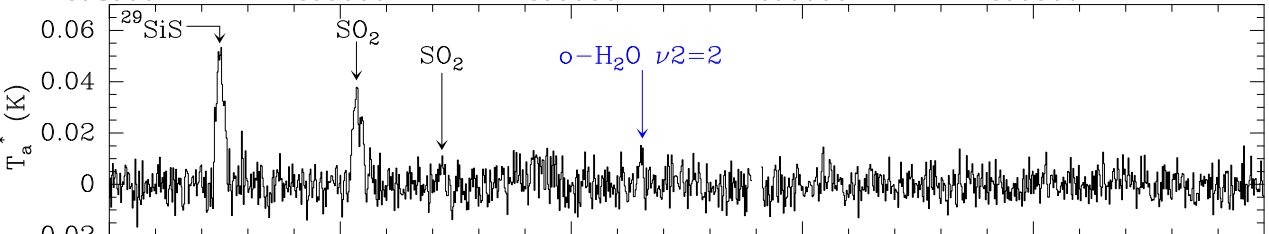

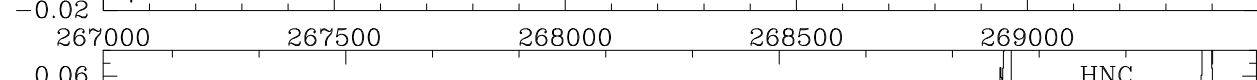

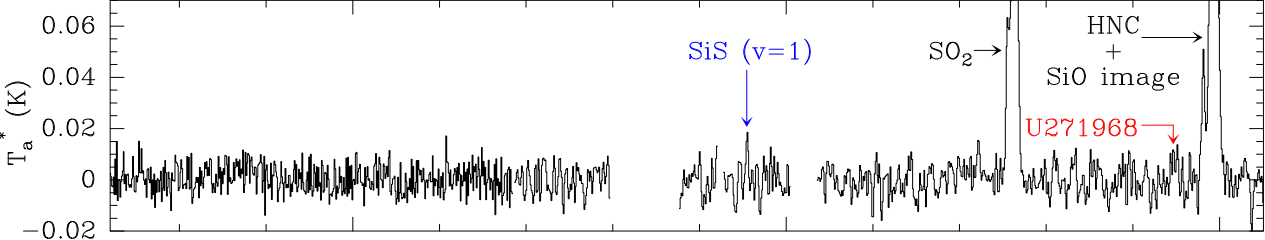

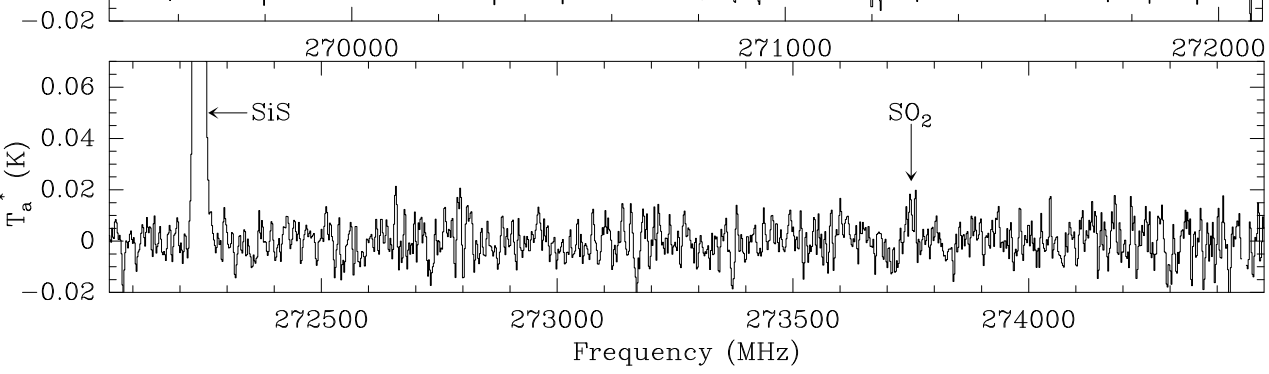

Fig. C.1. continued. 
L. Velilla Prieto et al.: The millimeter IRAM-30 m line survey toward IK Tauri
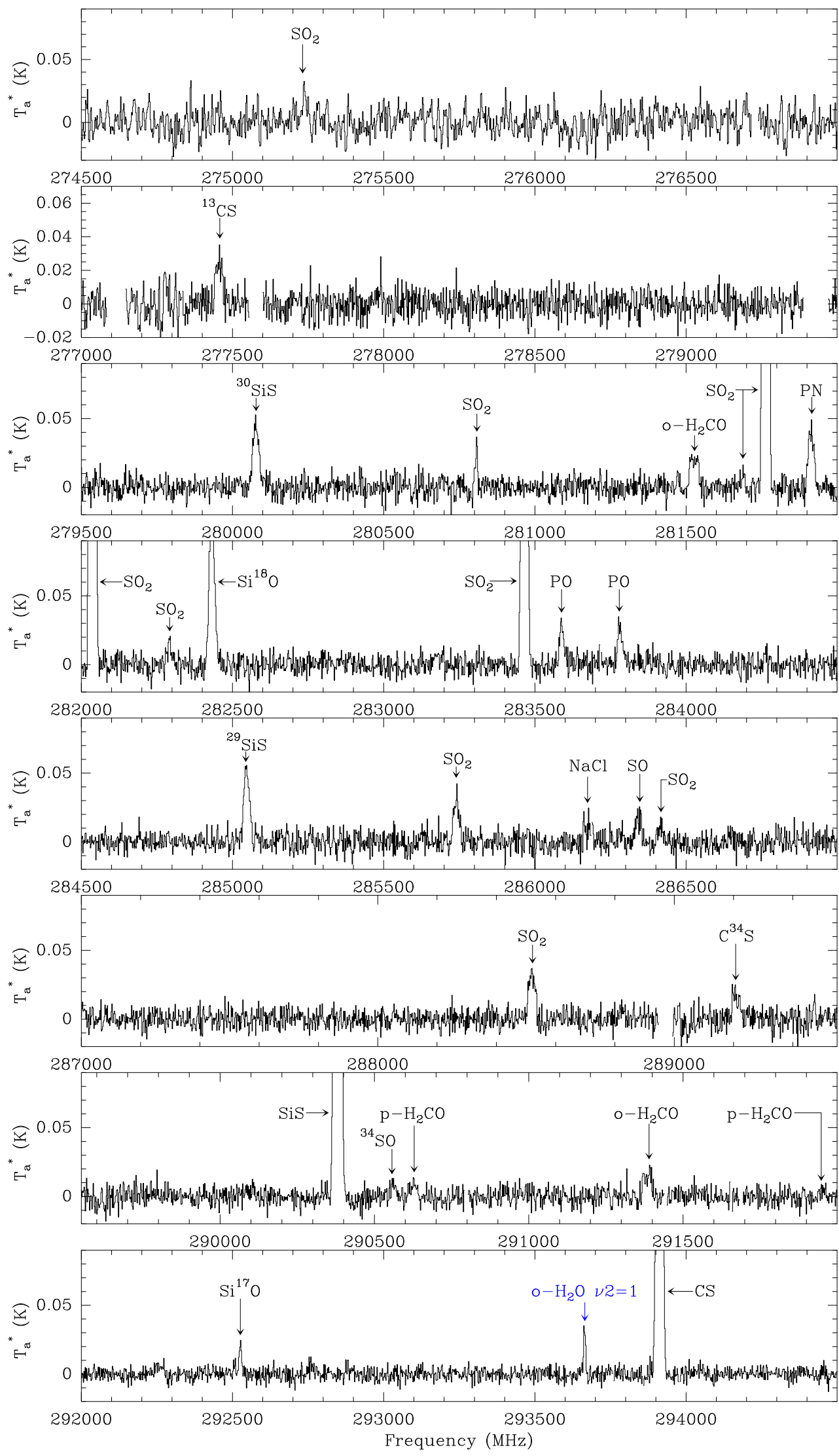

Fig. C.1. continued. 
A\&A 597, A25 (2017)
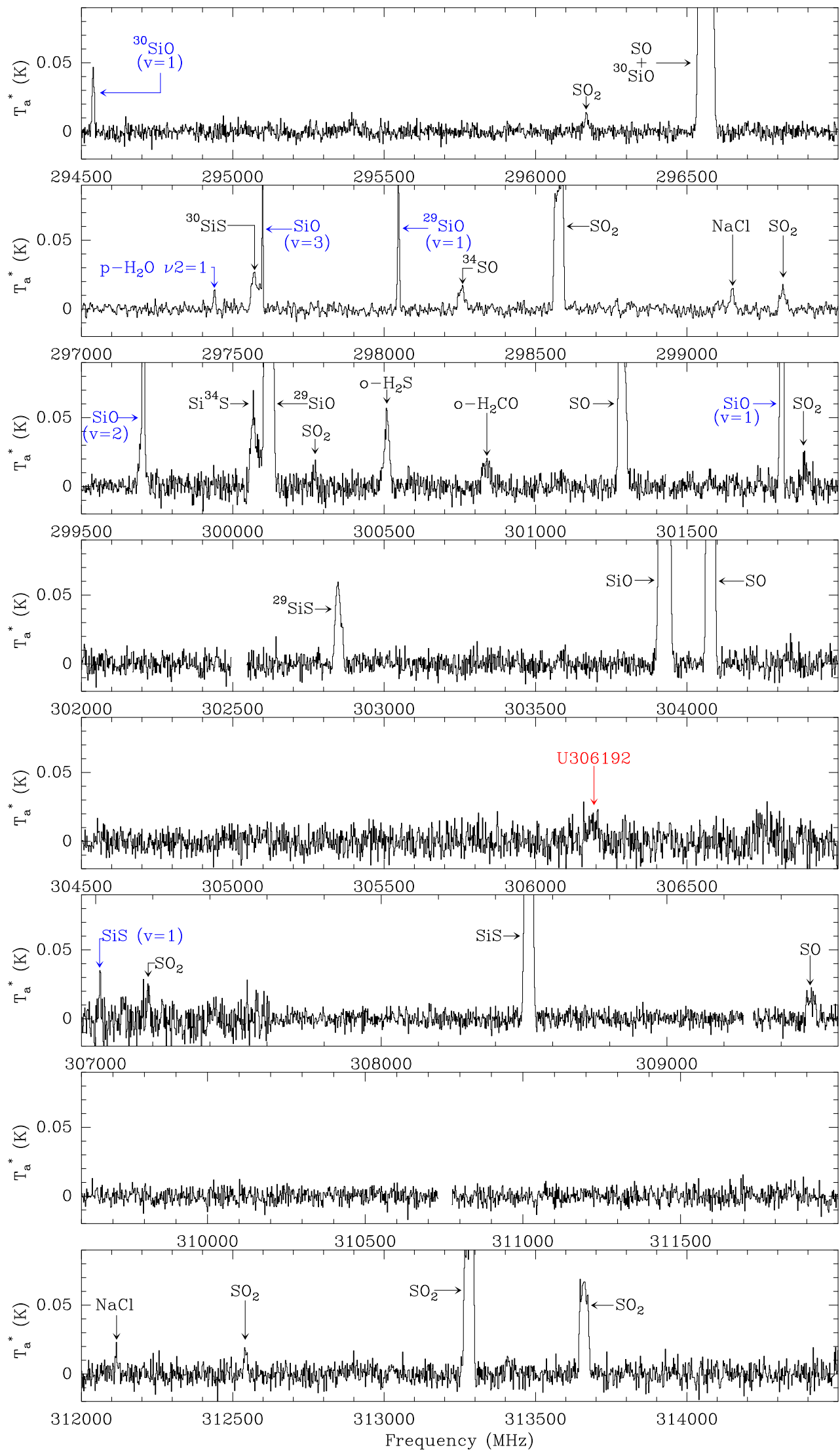

Fig. C.1. continued. 
L. Velilla Prieto et al.: The millimeter IRAM-30 m line survey toward IK Tauri
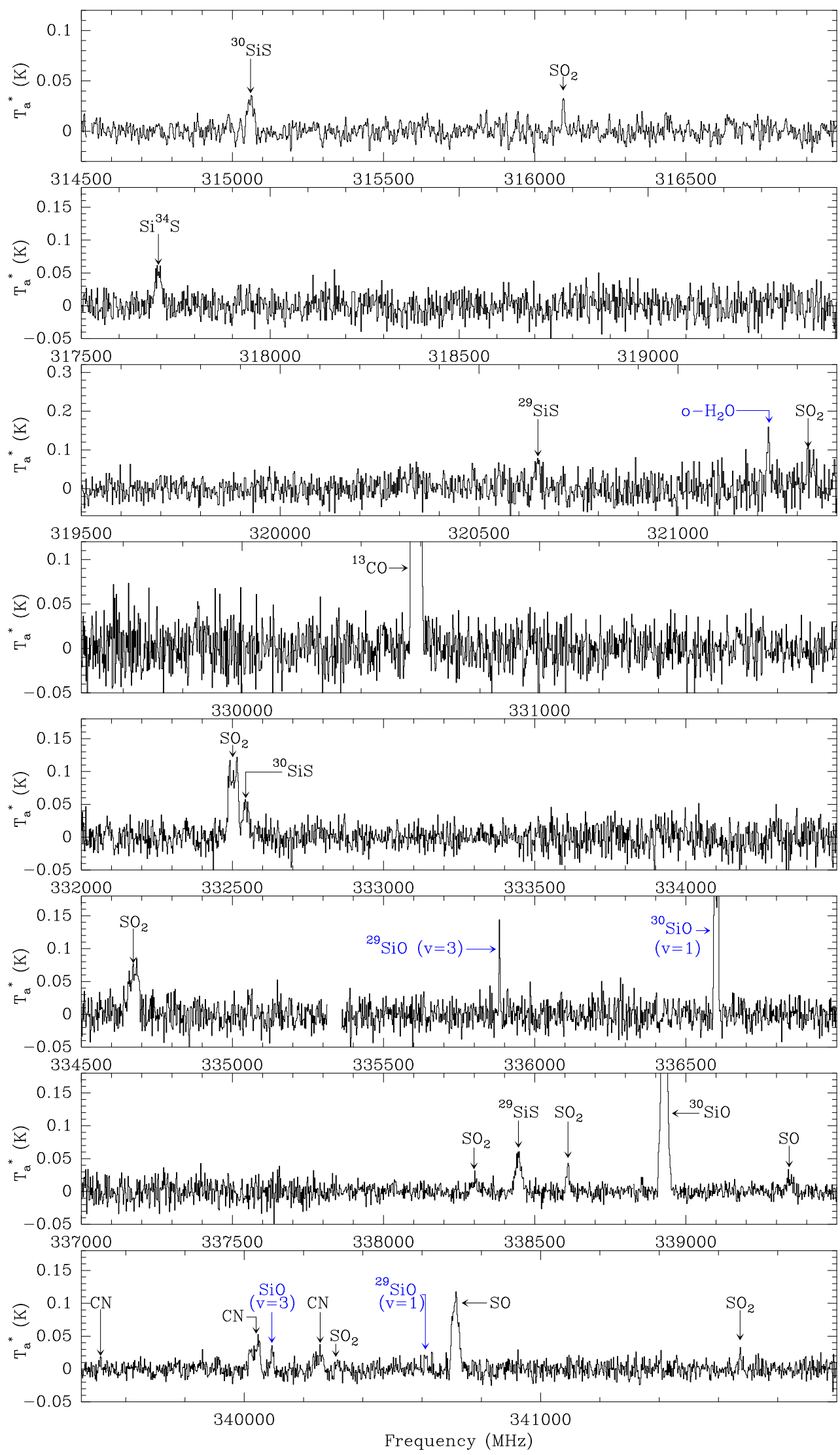

Fig. C.1. continued. 

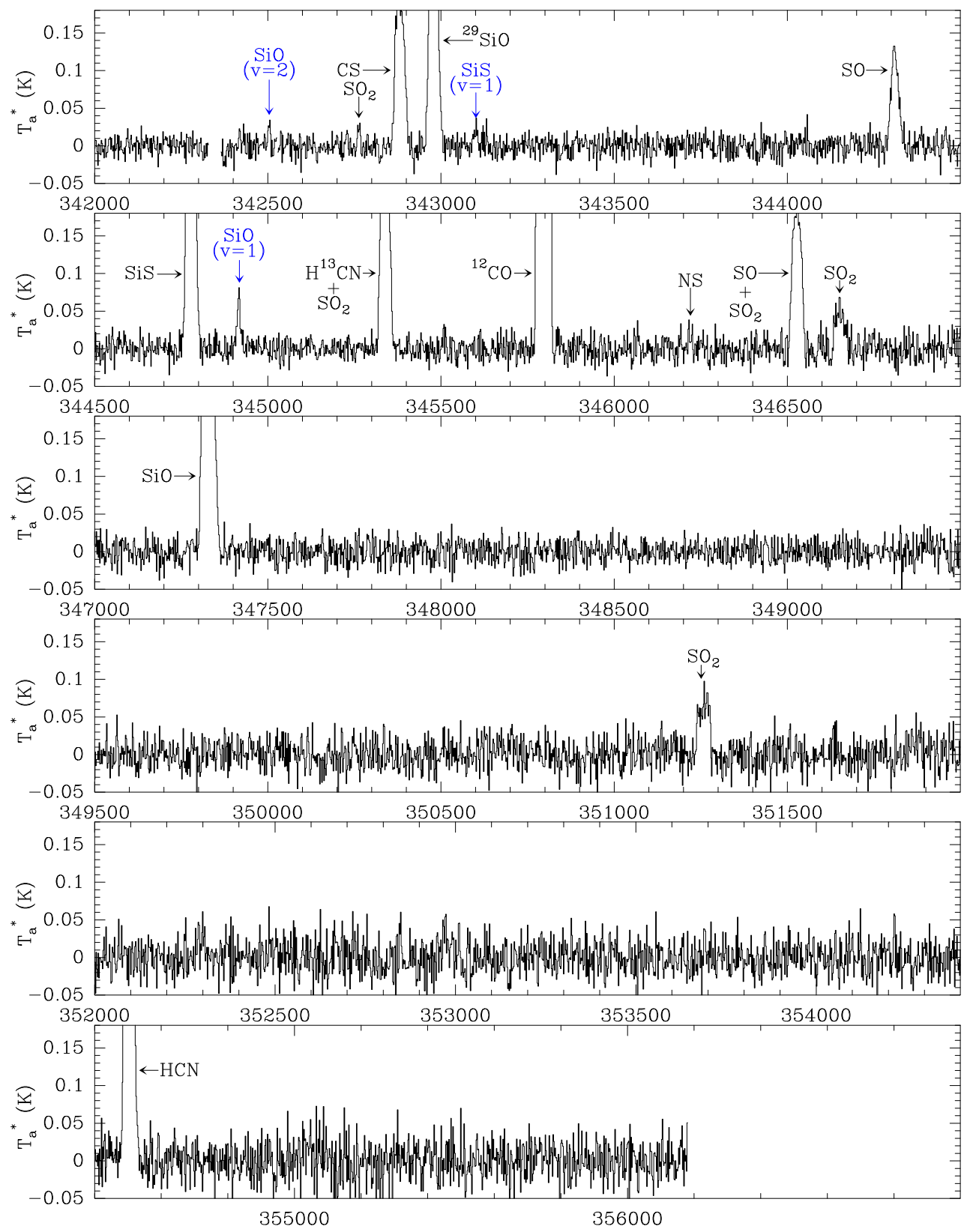

Frequency (MHz)

Fig. C.1. continued.

\section{Appendix D: Population diagrams}

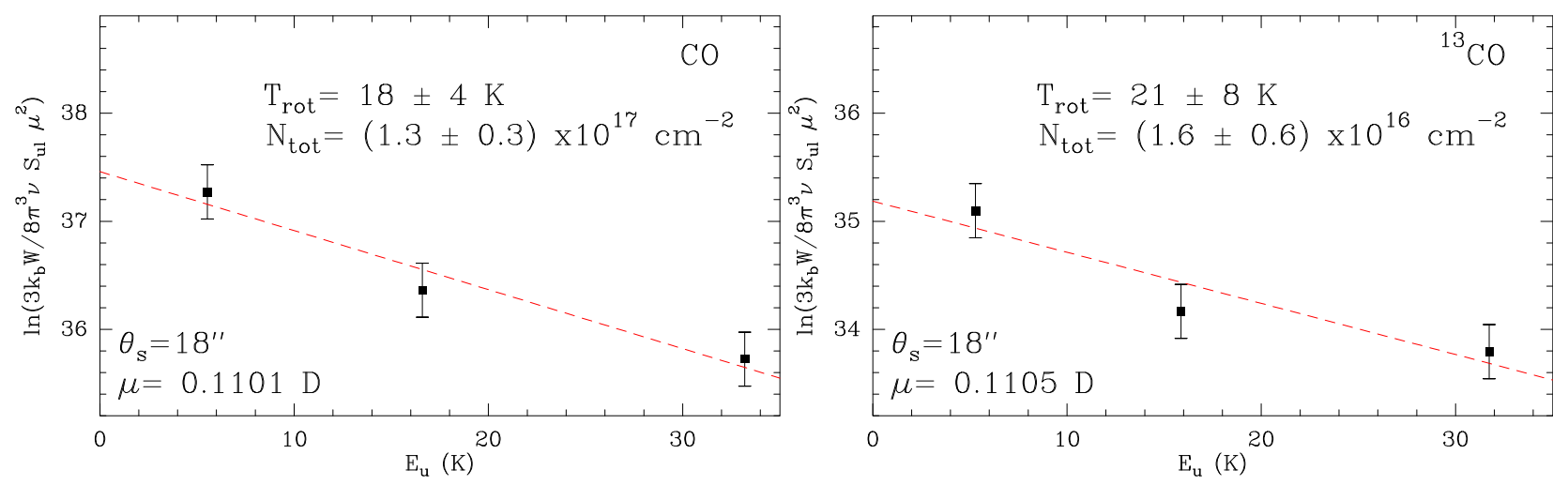

Fig. D.1. Population diagrams for $\mathrm{CO}$ and ${ }^{13} \mathrm{CO}$. The results from the fit (red dashed line) are shown in each box. The emission size adopted and the dipole moment of the molecule are shown in the bottom-left corner of each box. Error bars include the formal uncertainty of the measurement and a $25 \%$ uncertainty due to flux calibration or poor baseline substraction. 
L. Velilla Prieto et al.: The millimeter IRAM-30 m line survey toward IK Tauri

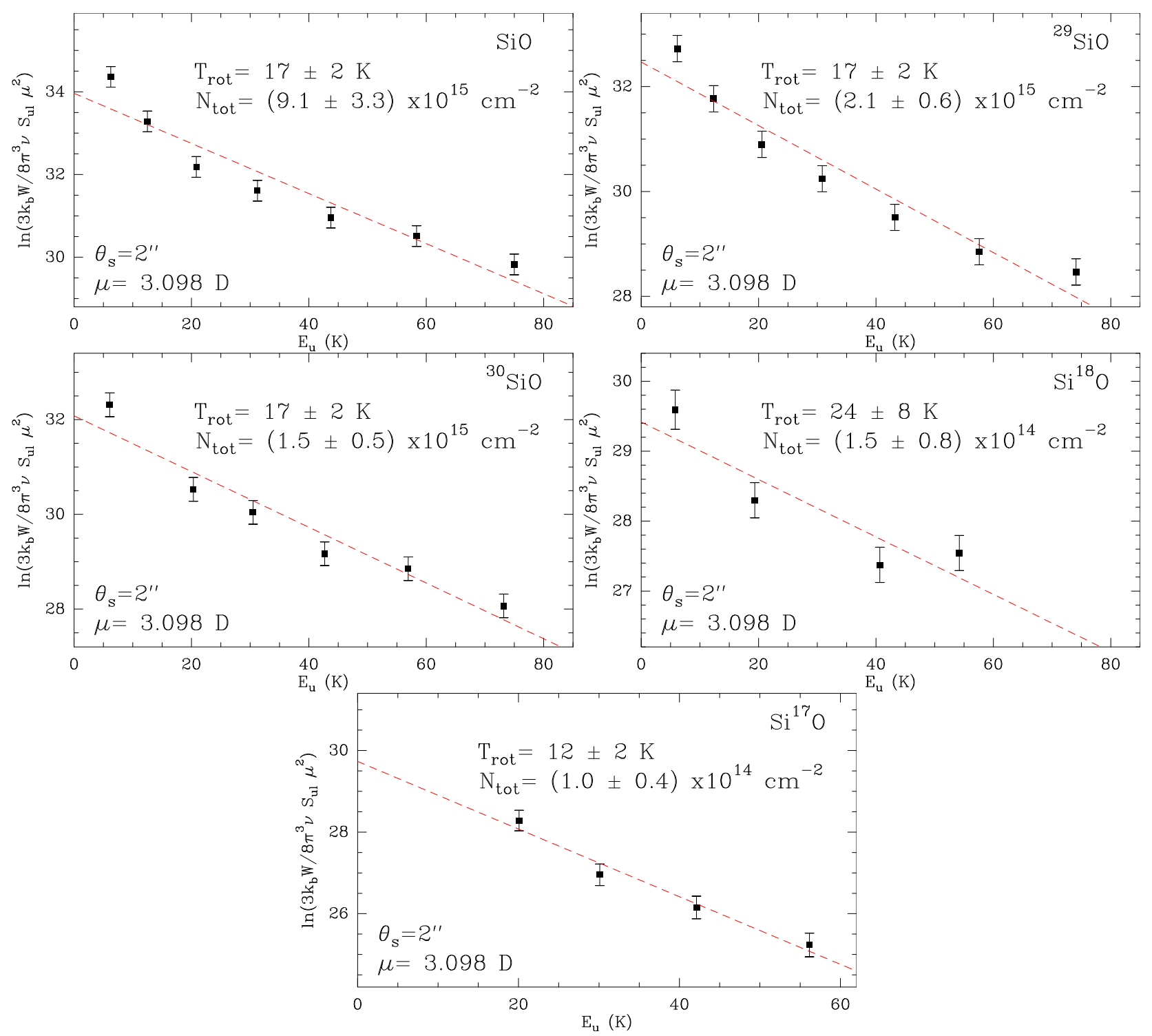

Fig. D.2. As in Fig. D.1 but for $\mathrm{SiO}$ isotopologues. 
A\&A 597, A25 (2017)
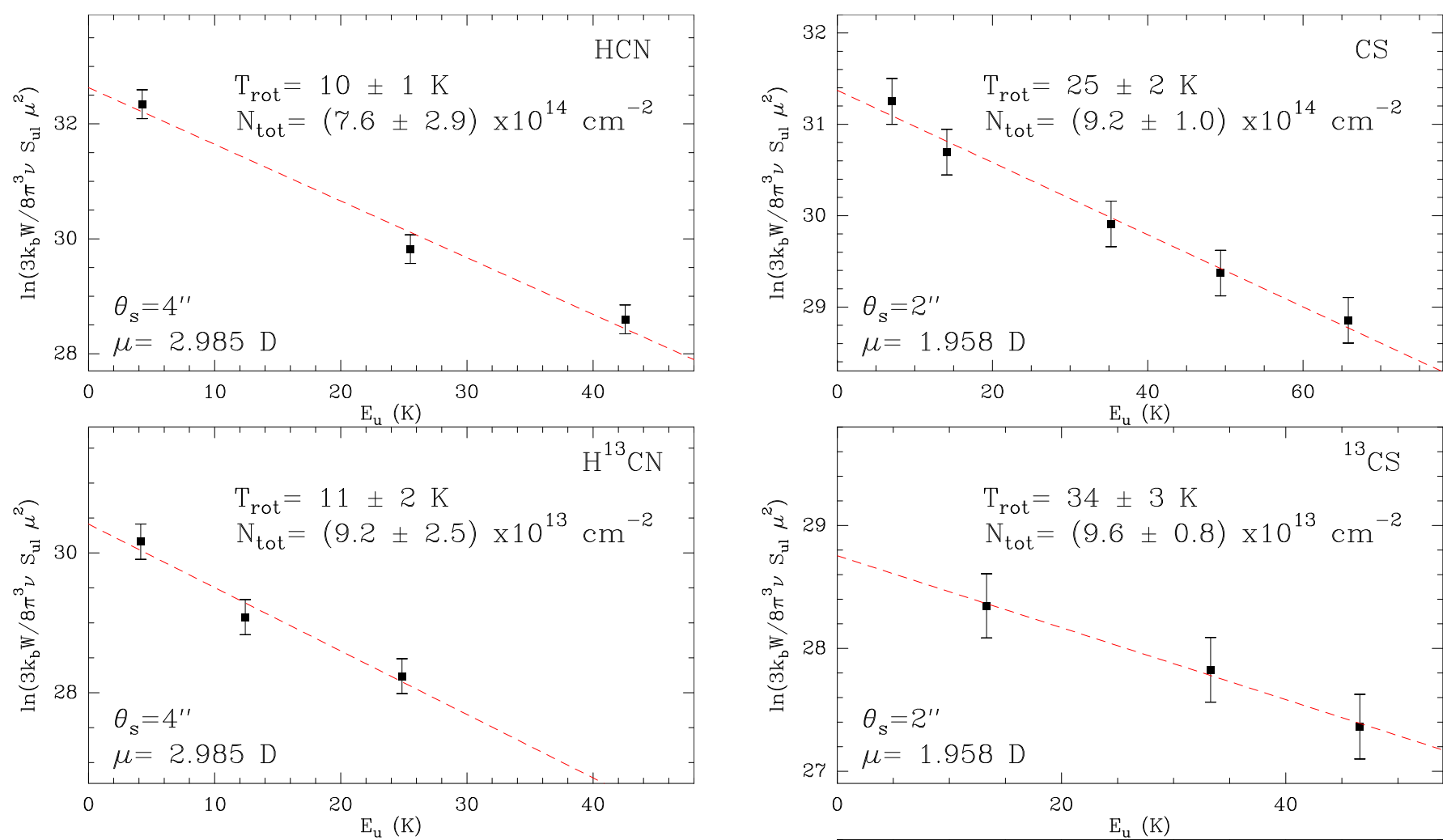

Fig. D.3. As in Fig. D.1 but for HCN isotopologues.

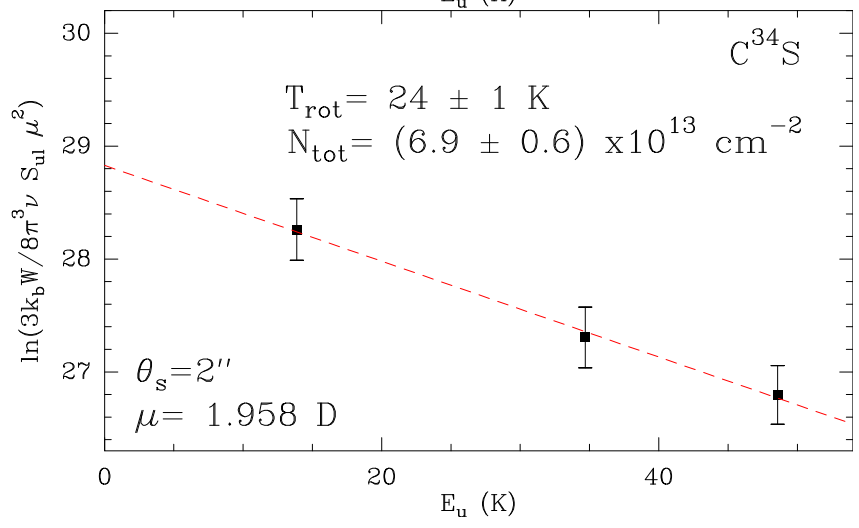

Fig. D.4. As in Fig. D.1 but for CS isotopologues. 
L. Velilla Prieto et al.: The millimeter IRAM-30 m line survey toward IK Tauri
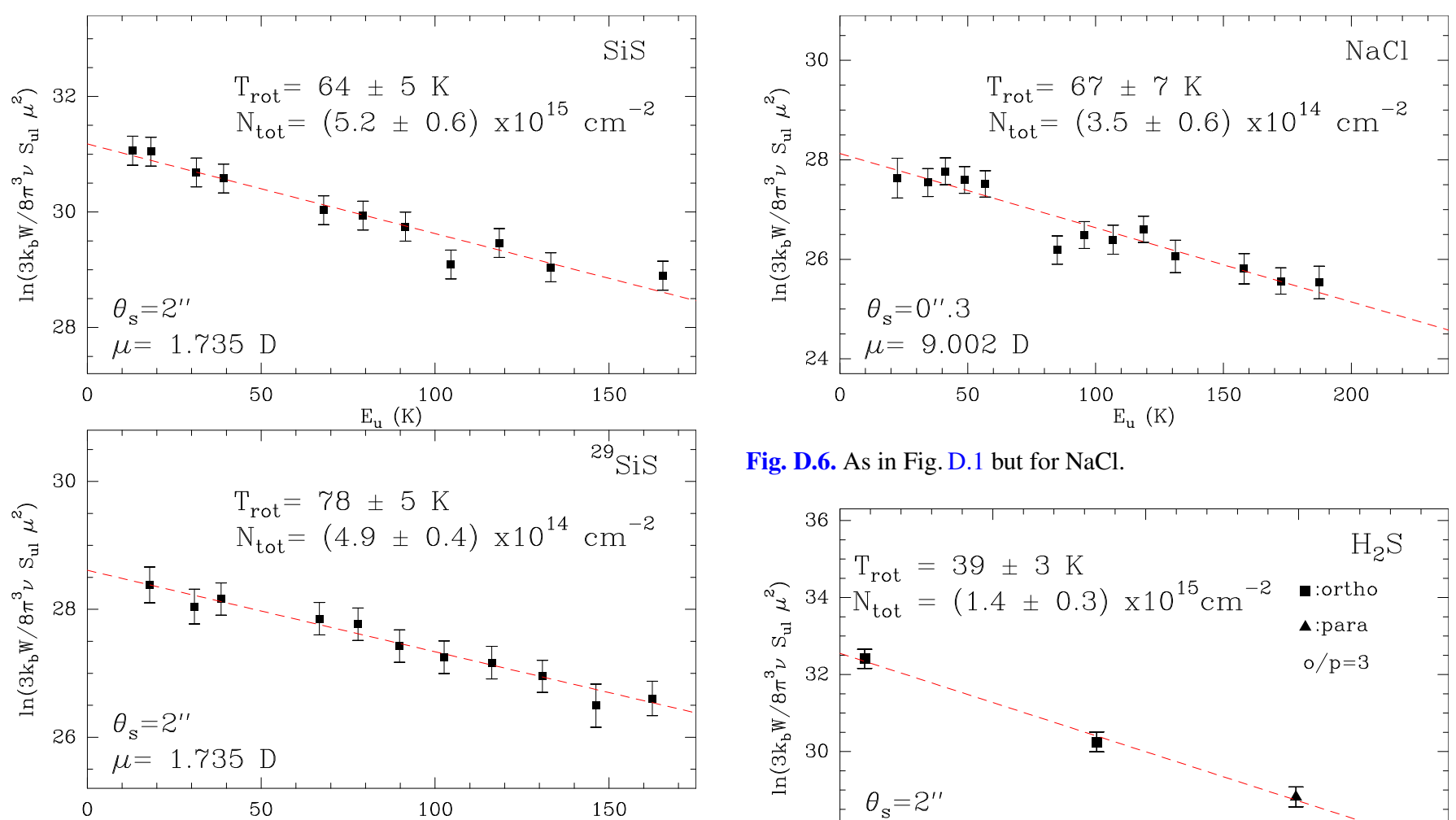

Fig. D.6. As in Fig. D. 1 but for $\mathrm{NaCl}$.

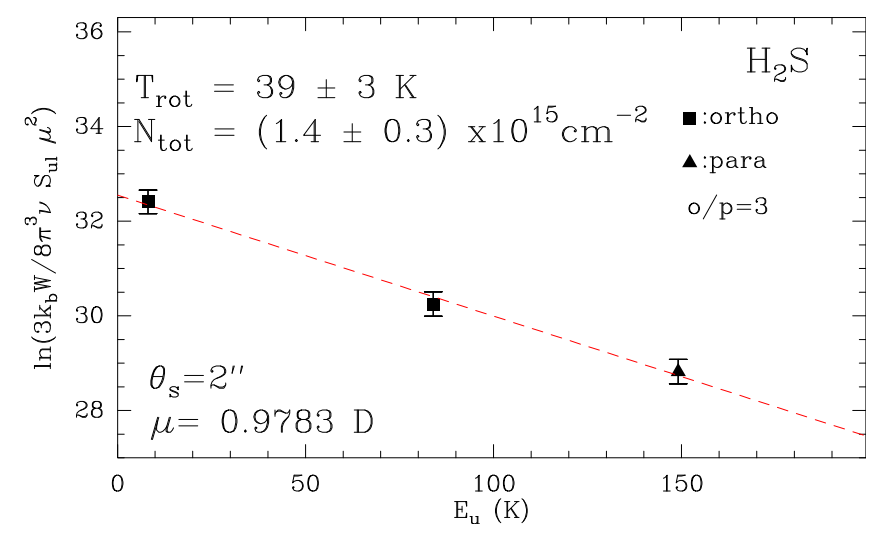

Fig. D.7. As in Fig. D. 1 but for $\mathrm{H}_{2} \mathrm{~S}$. We fitted simultaneously the ortho and para species adopting an ortho-to-para ratio of 3:1 (Decin et al. 2010a). The line o- $\mathrm{H}_{2} \mathrm{~S} J, K_{\mathrm{a}}, k=4_{1,4}-3_{2,1}$ significantly outlies the fit probably owing to its low $\mathrm{S} / \mathrm{N}$, therefore, it has been excluded from the diagram.

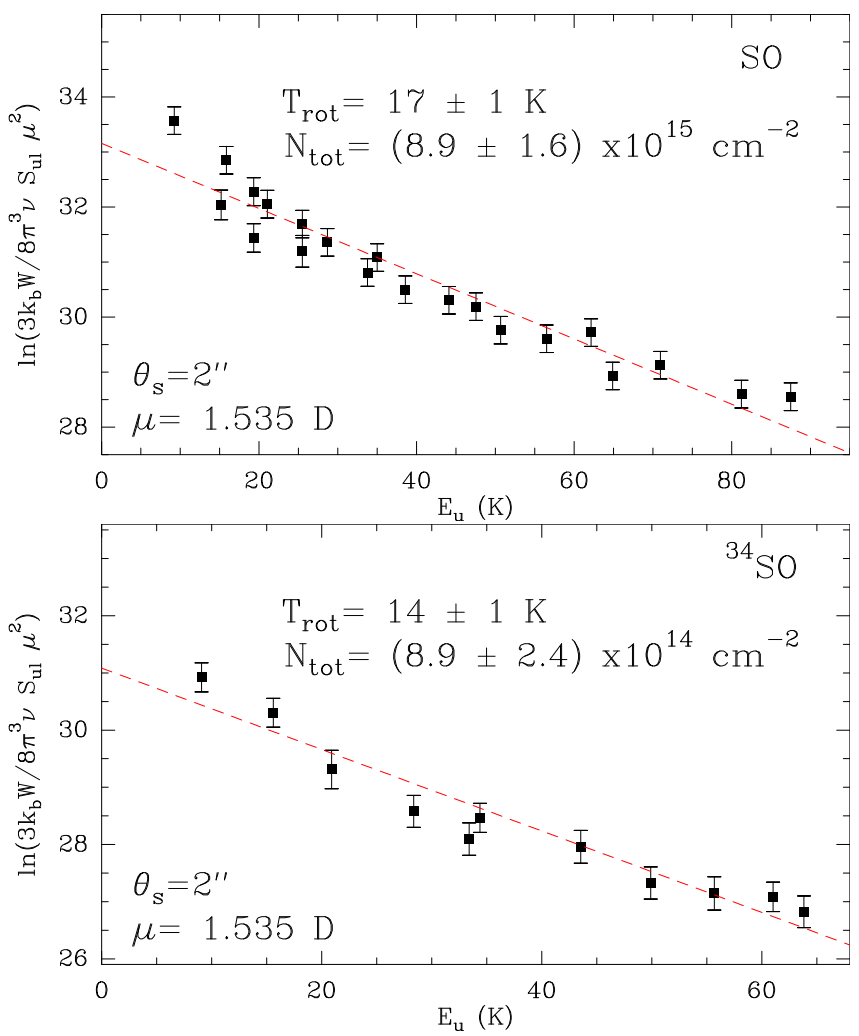

Fig. D.8. As in Fig. D.1 but for SO isotopologues. 


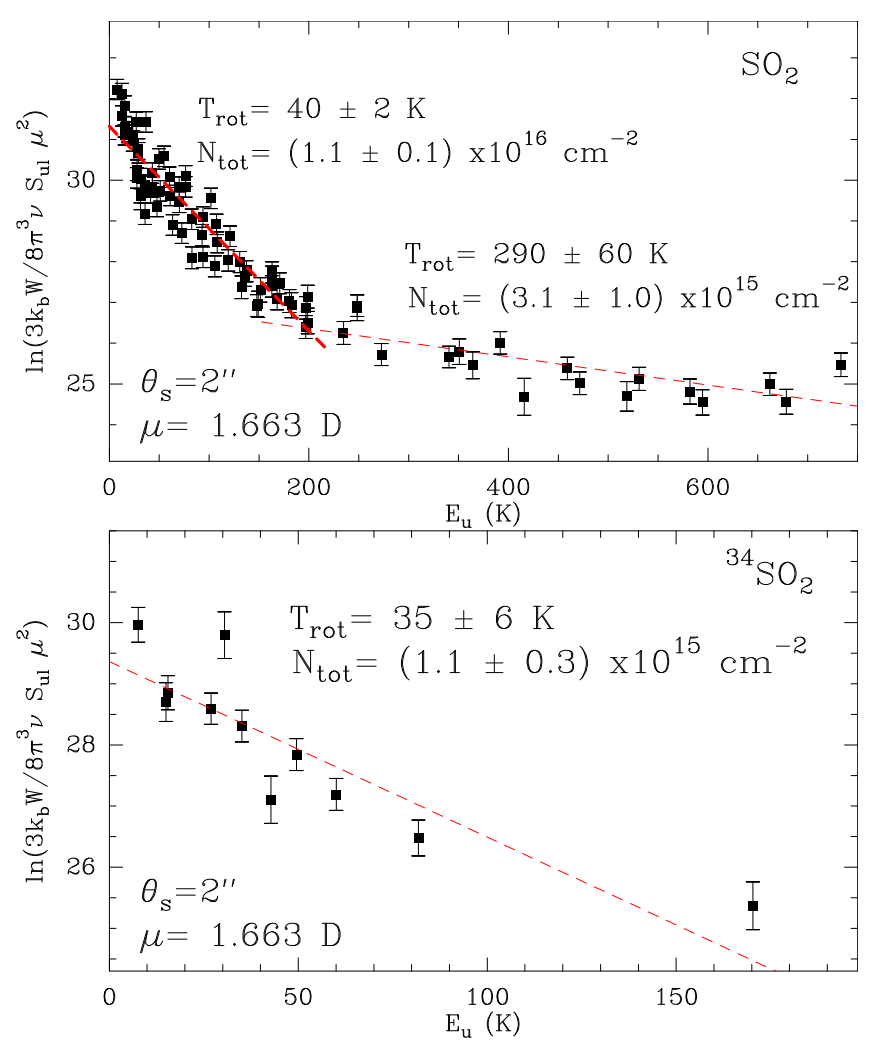

Fig. D.9. As in Fig. D. 1 but for $\mathrm{SO}_{2}$ isotopologues. The rotational diagram of $\mathrm{SO}_{2}$ was separated in two different trends (see Sect. 5.2).

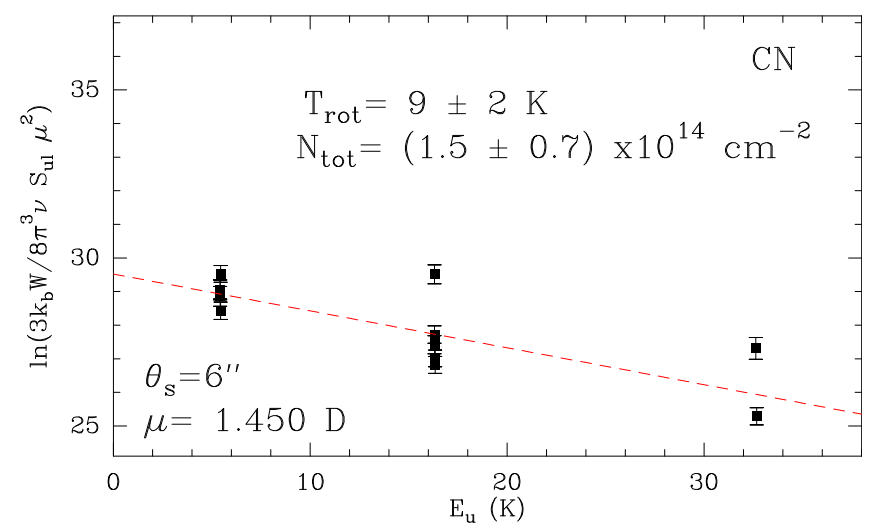

Fig. D.10. As in Fig. D.1 but for CN.

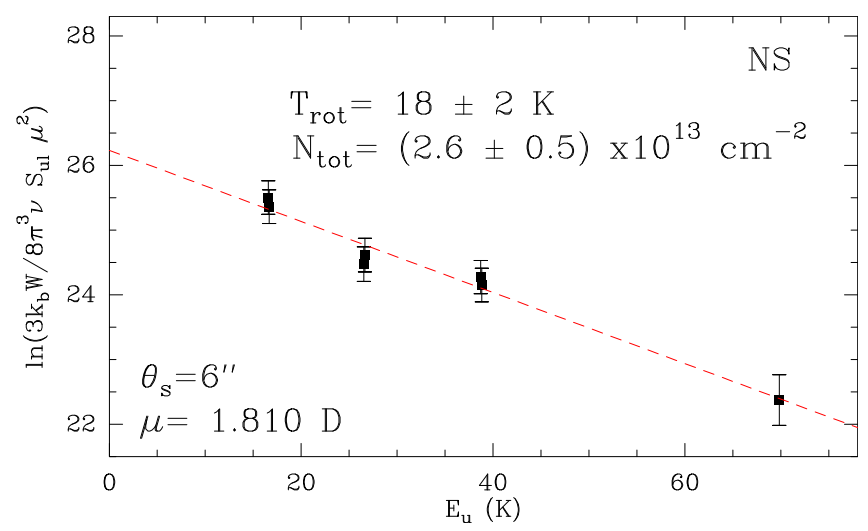

Fig. D.11. As in Fig. D.1 but for NS.

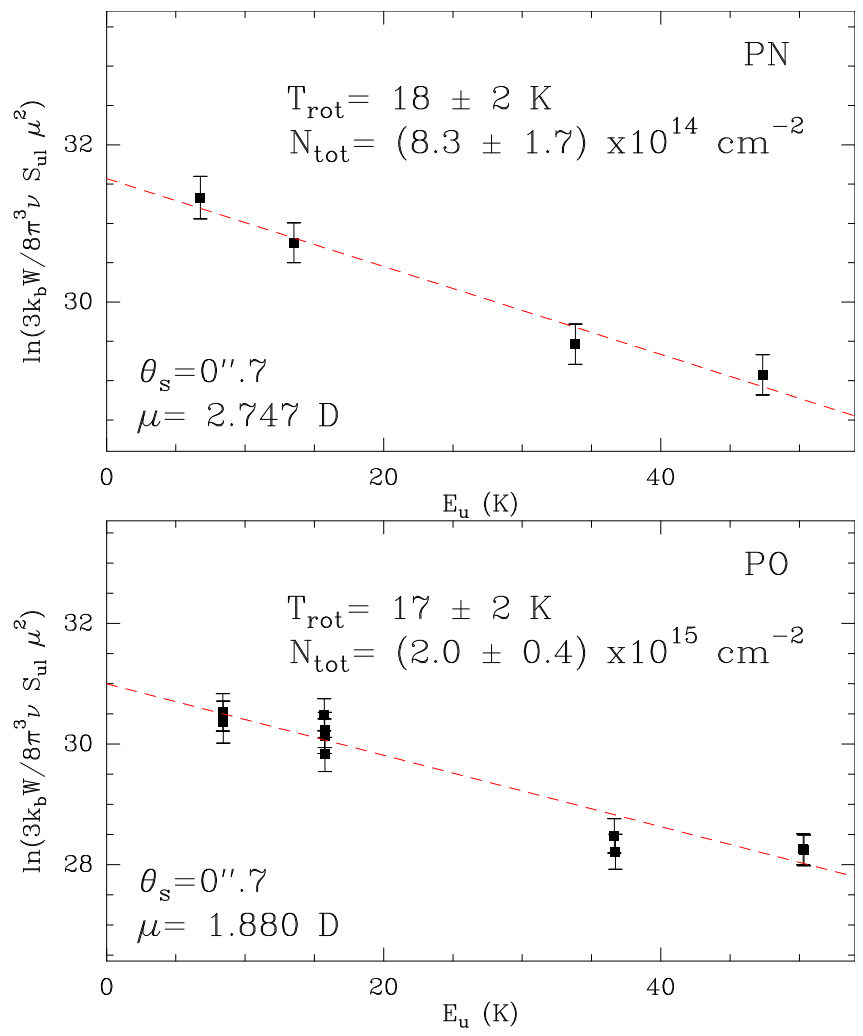

Fig. D.12. As in Fig. D. 1 but for P-bearing molecules. The PO line $\Omega=$ $1 / 2$, e, $J, F=(11 / 2,5)-(9 / 2,5)$ significantly outlies the fit probably owing to its low $\mathrm{S} / \mathrm{N}$, therefore, it has been excluded from the diagram.

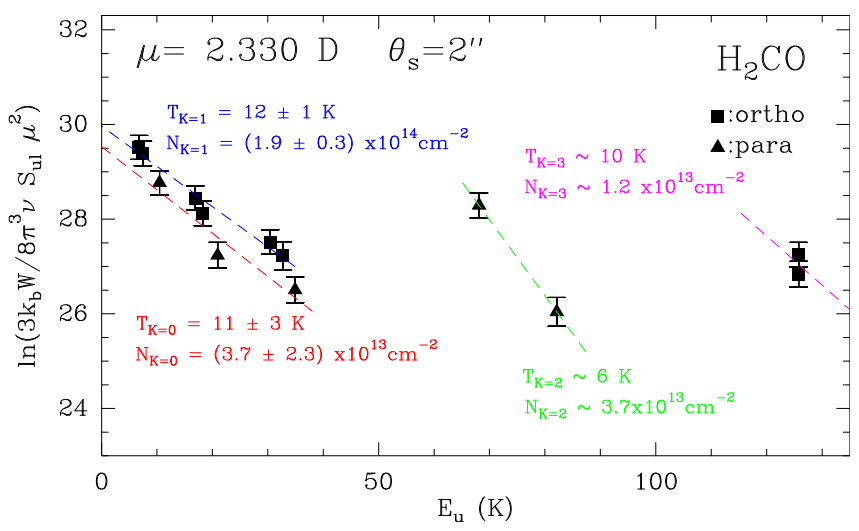

Fig. D.13. Population diagram for $\mathrm{H}_{2} \mathrm{CO}$. We show the results from the fits of the rotational ladders $K_{\mathrm{a}}=0$ (red), $K_{\mathrm{a}}=1$ (blue), and $K_{\mathrm{a}}=2$ (green). For the $K_{\mathrm{a}}=3$ (pink) we adopted as rotational temperature the average of the rotational temperatures of the $K_{\mathrm{a}}=0,1$, and 2 ladders to estimate a rough value for the $K_{\mathrm{a}}=3$ column density. 


\section{Appendix E: $\mathrm{SO}_{2}$ IOS calculations}

Close coupling (CC) calculations based on a state-of-the-art potential-energy surface (PES) (Spielfiedel et al. 2009) have provided collisional para- and ortho- $\mathrm{H}_{2}$ rate coefficients for temperatures ranging from 5 to $30 \mathrm{~K}$ for the 31 lowest $\mathrm{SO}_{2}$ rotational levels (Cernicharo et al. 2011). However, an extension of these full $\mathrm{CC}$ calculations to a large number of $\mathrm{SO}_{2}$ rotational levels and high temperatures would be prohibitive in terms of memory and CPU time, so the use of different approximate methods (coupled states (CS) and infinite order sudden (IOS)) is needed (Green 1976, 1979). Considering the high- $J \mathrm{SO}_{2}$ levels observed in the present work, we used the IOS approximation which is expected to give reliable estimates of rate coefficients, except for low energies. State-to-state de-excitation rate coefficients between the 410 lowest levels (with energy up to $743.9 \mathrm{~K}$ ) were calculated for energies up to $9000 \mathrm{~cm}^{-1}$ (value limited by the validity domain of the PES) giving data for temperatures up to $1000 \mathrm{~K}$ Balança et al. (in prep.). The $\mathrm{SO}_{2}$ energy levels and wave functions were obtained from spectroscopic constants describing the $\mathrm{SO}_{2}$ Hamiltonian up to the fourth order and provided by the MADEX code (Cernicharo 2012). The results show a relatively flat temperature variation of the rates.

The accuracy of these data was studied by comparing the resulting rate coefficients with $\mathrm{CC}$ and $\mathrm{CS}$ values obtained for collisions with para and ortho- $\mathrm{H}_{2}$ and all transitions involving the 31 lowest levels. Compared to CC and CS data, it is found that the IOS approach leads a systematic underestimation of para$\mathrm{H}_{2}$ rates, within a factor better than $50 \%$ in average, the agreement being better at high temperatures. However, the situation is not as good for collisions with ortho- $\mathrm{H}_{2}$ as the IOS approximation describes collisions with para- $\mathrm{H}_{2}\left(j_{\mathrm{H}_{2}}=0\right)$ and does take into account all the angular couplings involved in collisions with ortho- $\mathrm{H}_{2}$. The comparison between ortho- and para- $\mathrm{H}_{2} \mathrm{CS}$ rate coefficients shows that ortho- $\mathrm{H}_{2}$ rates are systematically larger than para- $\mathrm{H}_{2}$ rates by a factor two in average, with larger differences (up to a factor eight) for a number of transitions mainly identified as $\Delta K_{\mathrm{a}}=1$ transitions.
Table E.1. Parameters of the energy levels used for $\mathrm{SO}_{2}$ modelling.

\begin{tabular}{llllll}
\hline \hline Identifier & $J$ & $K_{\mathrm{a}}$ & $K_{\mathrm{c}}$ & $E$ & $g$ \\
- & - & - & - & $\mathrm{K}$ & - \\
\hline 1 & 0 & 0 & 0 & 0.000000 & 1 \\
2 & 2 & 0 & 2 & 2.751006 & 5 \\
3 & 1 & 1 & 1 & 3.339254 & 3 \\
4 & 2 & 1 & 1 & 5.320091 & 5 \\
5 & 3 & 1 & 3 & 7.743834 & 7 \\
6 & 4 & 0 & 4 & 9.151093 & 9 \\
$\ldots$ & & & & & \\
\hline
\end{tabular}

Notes. Table E.1, available at the CDS, contains the following information: Col. 1: identifier for the energy level used in Table E.2; Col. 2: quantum number $J$; Col. 3: quantum number $K_{\mathrm{a}}$; Col. 4: quantum number $K_{\mathrm{c}}$; Col. 5: energy of the level in Kelvin; Col. 6: degeneracy of the level.

The rate coefficients were implemented in MADEX fitting the logarithm of the rate coefficients to a sixth order polynomial as:

$P(x)=\sum_{i=0}^{6} a_{i} x^{i}$,

where

$x=T^{3 / 2}$,

which reproduces practically all the rates with a relative error below $30 \%$ for temperatures between 20 and $1000 \mathrm{~K}, 410$ energy levels and a maximum $E_{\mathrm{u}}=744 \mathrm{~K}$. We provide the energy levels involved in the calculations in Table E.1, as well as the polynomial coefficients of the fit in Table E.2. The level identifier used in Table E. 2 can be consulted in Table E. 1 to find the corresponding level. 
Table E.2. Polynomial coefficients of the fit to the rate coefficients.

\begin{tabular}{lllllllll}
\hline \hline Upper level id & Lower level id & $a_{0}$ & $a_{1}$ & $a_{2}$ & $a_{3}$ & $a_{4}$ & $a_{5}$ & $a_{6}$ \\
- & - & - & - & - & - & - & - & - \\
\hline 2 & 1 & -9.48415 & -29.3454 & 350.453 & -2071.21 & 6590.82 & -11012.6 & 7610.91 \\
3 & 1 & -9.58292 & -35.9159 & 406.891 & -2385.77 & 7513.84 & -12378.6 & 8410.64 \\
3 & 2 & -9.99698 & -14.3352 & 120.467 & -442.266 & 635.155 & 52.7172 & -657.401 \\
4 & 1 & 0.00000 & 0.00000 & 0.00000 & 0.00000 & 0.00000 & 0.00000 & 0.00000 \\
4 & 2 & -9.53669 & -26.9597 & 289.292 & -1598.36 & 4761.52 & -7413.75 & 4772.86 \\
4 & 3 & -9.09926 & -29.6351 & 346.972 & -2025.33 & 6401.05 & -10614.5 & 7267.38 \\
$\ldots$ & & & & & & & & \\
\hline
\end{tabular}

Notes. Table E.2, available at the CDS, contains the following information: Col. 1: identifier for the upper energy level described in Table E.1; Col. 2: identifier for the lower energy level described in Table E.1; Cols. 3-9: coefficients of the 6th order polynomial fit to the rate coefficients. 